\title{
Infinite dimensional multiplicity free spaces III: matrix coefficients and regular functions
}

\author{
Joseph A. Wolf
}

Received: 22 December 2009 / Revised: 31 March 2010 / Published online: 30 April 2010

C The Author(s) 2010. This article is published with open access at Springerlink.com

\begin{abstract}
In earlier papers we studied direct limits $(G, K)=\underset{\lim }{\longrightarrow}\left(G_{n}, K_{n}\right)$ of two types of Gelfand pairs. The first type was that in which the $\overrightarrow{G_{n}} / K_{n}$ are compact Riemannian symmetric spaces. The second type was that in which $G_{n}=N_{n} \rtimes K_{n}$ with $N_{n}$ nilpotent, in other words pairs $\left(G_{n}, K_{n}\right)$ for which $G_{n} / K_{n}$ is a commutative nilmanifold. In each we worked out a method inspired by the Frobenius-Schur Orthogonality Relations to define isometric injections $\zeta_{m, n}: L^{2}\left(G_{n} / K_{n}\right) \hookrightarrow L^{2}\left(G_{m} / K_{m}\right)$ for $m \geqq n$ and prove that the left regular representation of $G$ on the Hilbert space direct limit $L^{2}(G / K):=\lim _{\longrightarrow} L^{2}\left(G_{n} / K_{n}\right)$ is multiplicity-free. This left open questions concerning the nature of the elements of $L^{2}(G / K)$. Here we define spaces $\mathcal{A}\left(G_{n} / K_{n}\right)$ of regular functions on $G_{n} / K_{n}$ and injections $v_{m, n}: \mathcal{A}\left(G_{n} / K_{n}\right) \rightarrow \mathcal{A}\left(G_{m} / K_{m}\right)$ for $m \geqq n$ related to restriction by $\left.v_{m, n}(f)\right|_{G_{n} / K_{n}}=f$. Thus the direct limit $\mathcal{A}(G / K):=$ $\lim _{\longrightarrow}\left\{\mathcal{A}\left(G_{n} / K_{n}\right), v_{m, n}\right\}$ sits as a particular $G$-submodule of the much larger inverse limit $\lim _{\longleftarrow}\left\{\mathcal{A}\left(G_{n} / K_{n}\right)\right.$, restriction $\}$. Further, we define a pre Hilbert space structure on $\mathcal{A}(G / \overleftarrow{K})$ derived from that of $L^{2}(G / K)$. This allows an interpretation of $L^{2}(G / K)$ as the Hilbert space completion of the concretely defined function space $\mathcal{A}(G / K)$, and also defines a $G$-invariant inner product on $\mathcal{A}(G / K)$ for which the left regular representation of $G$ is multiplicity-free.
\end{abstract}

Mathematics Subject Classification (2000) Primary 22E65 · 17B65; Secondary $22 \mathrm{E} 70 \cdot 43 \mathrm{~A} 85$

Research partially supported by the National Science Foundation.

J. A. Wolf $(\varangle)$

Department of Mathematics, University of California,

Berkeley, CA 94720, USA

e-mail: jawolf@math.berkeley.edu 


\section{Introduction}

Gelfand pairs $(G, K)$, and the corresponding commutative homogeneous spaces $G / K$ generalize both the concept of Riemannian symmetric space and that of a familiar family of Riemannian nilmanifolds. Let $G$ be a locally compact topological group and $K$ a compact subgroup. Then $(G, K)$ is a Gelfand pair, i.e. $L^{1}(K \backslash G / K)$ is commutative under convolution, if and only if the (left regular) representation of $G$ on $L^{2}(G / K)$ is multiplicity free.

In two earlier notes $[17,18]$ we looked at certain cases where $G$ and $K$ are not locally compact, in fact are infinite dimensional of the form $\underset{\lim }{\longrightarrow}\left(G_{n}, K_{n}\right)$ where the $\left(G_{n}, K_{n}\right)$ are finite dimensional Gelfand pairs in the usual sense. We showed in those cases that the multiplicity-free condition is satisfied when $L^{2}(G / K)$ is interpreted as a certain Hilbert space direct limit of the usual $L^{2}\left(G_{n} / K_{n}\right)$. That direct limit made use of renormalizations inherent in the Frobenius-Schur othogonality relations for matrix coefficients of irreducible unitary representations, so the meaning of the elements of $L^{2}(G / K)$ was not immediate.

Here we define a ring of regular functions $\mathcal{A}(G / K)$ whose nature is transparent. It is a subalgebra of $\lim \mathcal{A}\left(G_{n} / K_{n}\right)$ where $\mathcal{A}\left(G_{n} / K_{n}\right)$ is the ring of regular functions in the usual sense and $\lim _{\longleftarrow} \mathcal{A}\left(G_{n} / K_{n}\right)$ is defined by restriction of functions. This is accompanied by a $G$-equivariant injective map $\mathcal{A}(G / K) \rightarrow L^{2}(G / K)$ with dense image. That leads to the main results of this note, both for the direct limits of compact symmetric spaces of [17] in Sect. 3 and for the direct limits of commutative nilmanifolds of [18] in Sects. 4 and 5. In both cases the results are that

$\mathcal{A}(G / K)$ injects to a dense subspace of $L^{2}(G / K)$, so $L^{2}(G / K)$ defines a $G$-invariant inner product on $\mathcal{A}(G / K)$, the regular representation of $G$ on $\mathcal{A}(G / K)$ is unitarized, and $L^{2}(G / K)$ can be interpreted as the Hilbert space completion of $\mathcal{A}(G / K)$.

\section{The ring of regular functions}

In this section we describe the general setup needed for constructing the ring of regular functions on direct systems of Lie groups and commutative spaces, and compare them with $L^{2}$ direct limits. We will specialize to parabolic direct systems of compact Lie groups compact Riemannian symmetric spaces in Sect. 3. Then we further specialize the results of this section to direct systems of certain classes of nilpotent Lie groups and commutative nilmanifolds in Sects. 4 and 5.

\subsection{Direct limit representations}

We consider direct limit groups $G=\lim _{\longrightarrow} G_{n}$ and direct limit unitary representations $\pi=\underline{\lim } \pi_{n}$ of them. This means that $\overrightarrow{\pi_{n}}$ is a unitary representation of $G_{n}$ on a Hilbert space $\overrightarrow{\mathcal{H}}_{\pi_{n}}$, that the $\mathcal{H}_{\pi_{n}}$ form a direct system whose maps are unitary, and that $\pi$ is 
the representation of $G$ on $\mathcal{H}_{\pi}=\underline{\lim } \mathcal{H}_{\pi_{n}}$ given by

$$
\begin{aligned}
\pi(g) v & =\pi_{n}\left(g_{n}\right) v_{n} \text { for } n \gg 0 \text { so that } V_{n} \hookrightarrow V \text { and } G_{n} \hookrightarrow G \text { send } v_{n} \\
& \rightarrow v \text { and } g_{n} \rightarrow g .
\end{aligned}
$$

This formal definition amounts to saying that $\pi$ is a well defined unitary representation of $G$ on $\mathcal{H}_{\pi}$.

If $u, v \in \mathcal{H}_{\pi_{n}}$ we have the matrix coefficient

$$
f_{u, v, n}: G_{n} \rightarrow \mathbb{C} \text { defined by } f_{u, v, n}(g)=\left\langle u, \pi_{n}(g) v\right\rangle_{\mathcal{H}_{\pi_{n}}}
$$

Since $\mathcal{H}_{\pi_{n}} \rightarrow \mathcal{H}_{\pi_{m}}$ is a $G_{n}$-equivariant unitary injection for $m \geqq n$, we may view $\mathcal{H}_{\pi_{n}}$ as a subspace of $\mathcal{H}_{\pi_{m}}$. This done, we have

$$
\left\langle u, \pi_{n}(g) v\right\rangle_{\mathcal{H}_{\pi_{n}}}=\left\langle u, \pi_{m}(g) v\right\rangle_{\mathcal{H}_{\pi_{m}}} \text { for } u, v \in \mathcal{H}_{\pi_{n}} \text { and } g \in G_{n},
$$

in other words $f_{u, v, n}=\left.f_{u, v, m}\right|_{G_{n}}$ for $u, v \in \mathcal{H}_{\pi_{n}}$. Now these coefficients form a direct system. Formally, we have

$$
\begin{aligned}
& \mathcal{A}\left(\pi_{n}\right):=\left\{\text { finite linear combinations of the } f_{u, v, n} \text { where } u, v \in \mathcal{H}_{\pi_{n}}\right\} \\
& \mathcal{A}\left(\pi_{n}\right) \hookrightarrow \mathcal{A}\left(\pi_{m}\right) \text { using }(2.3), \text { and } \mathcal{A}(\pi):=\lim _{\longrightarrow} \mathcal{A}\left(\pi_{n}\right) .
\end{aligned}
$$

In words, $\mathcal{A}\left(\pi_{n}\right)$ is the space of regular functions on $G_{n}$ defined by $\pi_{n}$ and $\mathcal{A}(\pi)$ is the space of regular functions on $G$ defined by $\pi$. They correspond to the idea of trigonometric polynomials inside $L^{2}$ of the circle, or more generally to the idea of Harish-Chandra module.

Note that $\mathcal{A}(\pi)$ is contained in the projective limit $\lim _{\longleftarrow} \mathcal{A}\left(\pi_{n}\right)$ (defined by restriction of functions), but it is much smaller.

\subsection{Square integrable direct limits}

From now on we assume that the groups $G_{n}$ are separable, unimodular, locally compact and of Type I. (We will be dealing with commutative spaces $G_{n} / K_{n}$, and the commutativity implies unimodularity for $G_{n}$ ). Then one has the classical decomposition

$$
L^{2}\left(G_{n}\right)=\int_{\widehat{G_{n}}}\left(\mathcal{H}_{\pi_{n}} \widehat{\otimes} \mathcal{H}_{\pi_{n}}^{*}\right) d\left(\pi_{n}\right)
$$

where $d\left(\pi_{n}\right)$ is Plancherel measure on the unitary dual $\widehat{G_{n}}$ and the projective tensor product $\mathcal{H}_{\pi_{n}} \widehat{\otimes} \mathcal{H}_{\pi_{n}}^{*}$ contains $\mathcal{A}\left(\pi_{n}\right)$ as a dense subspace. This expresses a function $f \in L^{1}\left(G_{n}\right) \cap L^{2}\left(G_{n}\right)$ in the form $f(g)=\int_{\widehat{G}_{n}} \pi_{n}(f) d\left(\pi_{n}\right)$ where $\pi_{n}(f)=$ $\int_{G_{n}} f(g) \pi_{n}(g) d g \in \mathcal{H}_{\pi_{n}} \widehat{\otimes} \mathcal{H}_{\pi_{n}}^{*}$ is a Hilbert-Schmidt operator on $\mathcal{H}_{\pi_{n}}$ and where one has the Plancherel formula $\|f\|_{2}^{2}=\int_{\widehat{G}_{n}}\left\|\pi_{n}(f)\right\|_{H S}^{2} d\left(\pi_{n}\right)$. 
Fix a closed subgroup $Z_{n}$ in $G_{n}$ that is co-compact in the center. It can be $\{1\}$ if $G_{n}$ has compact center. If $\chi \in \widehat{Z_{n}}$ let $\widehat{G_{n, \chi}}=\left\{\pi_{n} \in \widehat{G_{n}} \mid \pi_{n}(x z)=\chi(z)^{-1} \pi_{n}(x)\right\}$ and consider the Hilbert space $L^{2}\left(G_{n, \chi}\right)$ of $L^{2}$ sections of the $G_{n}$-bundle $\left(G_{n} \times_{\chi} \mathbb{C}\right) \rightarrow$ $G_{n} / Z_{n}$. Then

$$
L^{2}\left(G_{n, \chi}\right)=\underset{\widehat{G}_{n, \chi}}{\int}\left(\mathcal{H}_{\pi_{n}} \widehat{\otimes} \mathcal{H}_{\pi_{n}}^{*}\right) d_{n, \chi}\left(\pi_{n}\right)
$$

where $d_{n, \chi}\left(\pi_{n}\right)$ is Plancherel measure on $\widehat{G_{n, \chi}}$. The decomposition (2.5) then decomposes further into

$$
L^{2}\left(G_{n}\right)=\int_{\widehat{Z_{n}}} L^{2}\left(G_{n, \chi}\right) d_{n}(\chi)
$$

An irreducible unitary representation $\pi_{n} \in \widehat{G_{n}}$ is called square integrable if its coefficients $f_{u, v, n}$ satisfy $\left|f_{u, v, n}\right| \in L^{2}\left(G_{n} / Z_{n}\right)$. This makes sense because $Z_{n}$ is co-compact in the center of $G_{n}$. Then one has the Godement-Frobenius-Schur orthogonality relations. In particular there is a number $\operatorname{deg} \pi_{n}>0$ (called the formal degree) such that

$$
\left\|f_{u, v, n}\right\|_{L^{2}\left(G_{n} / Z_{n}\right)}^{2}=\frac{1}{\operatorname{deg} \pi_{n}}\|u\|_{\mathcal{H}_{\pi_{n}}}^{2}\|v\|_{\mathcal{H}_{\pi_{n}}}^{2} .
$$

Now suppose that we have the $\widehat{G_{n}}$ arranged so that we have direct systems $\left\{\pi_{n}\right\}$ of unitary representations and, for each $n$, every $\pi_{n} \in \widehat{G_{n}}$ belongs to exactly one of those direct systems. Write $\beta_{m, n, \pi}: \mathcal{H}_{\pi_{n}} \rightarrow \mathcal{H}_{\pi_{m}}$ for the direct system maps on the representation spaces. They are unitary. For each such direct system we have the direct limit unitary representation $\pi=\lim \pi_{n}$ of $G=\lim G_{n}$ and the direct limit representation space $\mathcal{H}_{\pi}=\underset{\lim }{\longrightarrow}\left\{\mathcal{H}_{\pi_{n}}, \beta_{m, n, \pi}\right\}$.

We now make two strong assumptions on the system $\left\{G_{n}\right\}$. First, suppose that we can (and do) choose the co-compact closed central subgroups $Z_{n} \subset G_{n}$ so that

$$
G_{n} \hookrightarrow G_{n+1} \text { maps } Z_{n} \cong Z_{n+1} .
$$

Then we can write $Z$ for each of the groups $Z_{n}$, and each of the direct systems $\left\{\pi_{n}\right\}$ has a common central character $\chi \in \widehat{Z}$. We now make the further assumption on the groups $G_{n}$ that

Plancherel-almost-all $\pi_{n} \in \widehat{G_{n}}$ are square integrable: their $\left|f_{u, v, n}\right| \in L^{2}\left(G_{n} / Z\right)$.

As in [17] and [18] we use (2.8) and (2.10) to scale the inclusions $\mathcal{H}_{\pi_{n}} \widehat{\otimes} \mathcal{H}_{\pi_{n}}^{*} \hookrightarrow$ $\mathcal{H}_{\pi_{m}} \widehat{\otimes} \mathcal{H}_{\pi_{m}}^{*}$ for $m \geqq n$ by means of formal degrees and obtain $\left(G_{n} \times G_{n}\right)$-equivariant 
isometric inclusions

$$
\zeta_{m, n, \pi}: \mathcal{H}_{\pi_{n}} \widehat{\otimes} \mathcal{H}_{\pi_{n}}^{*} \rightarrow \mathcal{H}_{\pi_{m}} \widehat{\otimes} \mathcal{H}_{\pi_{m}}^{*} \text { defined by } f_{u, v, n} \mapsto\left(\frac{\operatorname{deg} \pi_{m}}{\operatorname{deg} \pi_{n}}\right)^{1 / 2} f_{u, v, m}
$$

In other words $\zeta_{m, n, \pi}=\frac{\operatorname{deg} \pi_{m}}{\operatorname{deg} \pi_{n}}\left(\beta_{m, n, \pi} \otimes \beta_{m, n, \pi^{*}}\right)$. That gives us direct systems of Hilbert spaces and the direct limits

$$
\mathcal{H}_{\pi}=\lim _{\longrightarrow}\left\{\mathcal{H}_{\pi_{n}}, \beta_{m, n, \pi}\right\} \text { and } \mathcal{H}_{\pi} \widehat{\otimes} \mathcal{H}_{\pi}^{*}=\lim _{\longrightarrow}\left\{\left(\mathcal{H}_{\pi_{n}} \widehat{\otimes} \mathcal{H}_{\pi_{n}}^{*}\right), \zeta_{m, n, \pi}\right\}
$$

of representation spaces for the irreducible unitary representations $\pi=\underline{\lim } \pi_{n}$ of $G$ and $\pi \otimes \pi^{*}$ of $G \times G$.

In order to sum the $\mathcal{H}_{\pi_{n}} \widehat{\otimes} \mathcal{H}_{\pi_{n}}^{*}$ and the $\mathcal{H}_{\pi} \widehat{\otimes} \mathcal{H}_{\pi}^{*}$ as in (2.6), to form $L^{2}\left(G_{\chi}\right)=$ $\lim _{\longrightarrow} L^{2}\left(G_{n, \chi}\right)$, we need the direct integral of (2.6) to be consistent with the rescaling

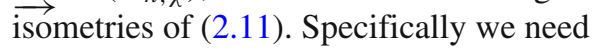

$$
\begin{aligned}
& \int_{\widehat{G_{n, \chi}}}\left(\mathcal{H}_{\pi_{n}} \widehat{\otimes} \mathcal{H}_{\pi_{n}}^{*}\right) d_{n, \chi}\left(\pi_{n}\right) \quad \stackrel{\int_{\widehat{G_{n, \chi}}} \zeta_{m, n, \pi} d_{n, \chi}\left(\pi_{n}\right)}{\longrightarrow} \int_{\widehat{G_{m, \chi}}}\left(\mathcal{H}_{\pi_{m}} \widehat{\otimes} \mathcal{H}_{\pi_{m}}^{*}\right) d_{m, \chi}\left(\pi_{m}\right) \\
& \downarrow \int_{\widehat{G_{n, \chi}}} \zeta_{m, n, \pi} d_{n, \chi}\left(\pi_{n}\right) \\
& \int_{\widehat{G_{n, \chi}}} \zeta_{m, n, \pi}\left(\mathcal{H}_{\pi_{n}} \widehat{\otimes} \mathcal{H}_{\pi_{n}}^{*}\right) d_{n, \chi}\left(\pi_{n}\right) \quad \longrightarrow \quad \int_{\widehat{G_{m, \chi}}}\left(\mathcal{H}_{\pi_{m}} \widehat{\otimes} \mathcal{H}_{\pi_{m}}^{*}\right) d_{m, \chi}\left(\pi_{m}\right)
\end{aligned}
$$

to commute for $m \geqq n$. In the cases studied here, the $\widehat{G_{n, \chi}}$ are discrete (or even finite), so the $L^{2}\left(G_{n, \chi}\right)$ are discrete direct sums of irreducible representations. Then there is no consistency problem with the rescaling isometries: one simply takes their discrete direct sum.

Once the $\mathcal{H}_{\pi_{n}} \widehat{\otimes} \mathcal{H}_{\pi_{n}}^{*}$ have been summed as in (2.6), we need to control the integration over the $\widehat{Z}$ in (2.7) in order to pass to the limit and form $L^{2}(G)=\int_{\widehat{Z}} L^{2}\left(G_{\chi}\right) d(\chi)$. Here we need the conditions of (2.9), that every $Z_{n}=Z$, and then we need that

for $m, n \gg 0$ the measures $d_{m}$ and $d_{n}$ on $\widehat{Z}$ are mutually absolutely continuous.

In the cases studied here condition (2.14) will be easy to verify.

\subsection{Construction of $\mathcal{A}(G)$}

Our hypothesis (2.10) of square integrability says that Plancherel-almost-all $\mathcal{A}\left(\pi_{n}\right) \subset$ $\mathcal{H}_{\pi_{n}} \widehat{\otimes} \mathcal{H}_{\pi_{n}}^{*}$, in fact form a dense subspace there. We want the summation $L^{2}\left(G_{n, \chi}\right)=$ $\int_{G_{n, \chi}}\left(\mathcal{H}_{\pi_{n}} \widehat{\otimes} \mathcal{H}_{\pi_{n}}^{*}\right) d_{n, \chi}\left(\pi_{n}\right)$ to restrict to a summation of the $\mathcal{A}\left(\pi_{n}\right)$ and form a space $\mathcal{A}\left(G_{n, \chi}\right)$ consisting of regular functions on $G_{n}$ that transform by $\chi$. In order to do this we must look at the detailed definition of direct integral. 
Definition 2.15 Let $(Y, \mathcal{M}, \tau)$ be a measure space. For each $y \in Y$ let $\mathcal{H}_{y}$ be a separable Hilbert space. Fix a family $\left\{s_{\alpha}\right\}_{\alpha \in A}$ of maps $Y \rightarrow \bigcup_{y \in Y} \mathcal{H}_{y}$ such that

(i) $s_{\alpha}(y) \in \mathcal{H}_{y}$ a.e. $(Y, \mathcal{M}, \tau)$, for all $\alpha \in A$,

(ii) $y \mapsto\left\langle s_{\alpha}(y), s_{\beta}(y)\right\rangle_{\mathcal{H}_{y}}$ belongs to $L^{1}(Y, \tau)$, for all $\alpha, \beta \in A$, and

(iii) $\mathcal{H}_{y}$ is the closed span of $\left\{s_{\alpha}(y)\right\}_{\alpha \in A}$ a.e. $(Y, \tau)$.

Then the (Hilbert space) direct integral defined by the measure space $(Y, \mathcal{M}, \tau)$, the family $\left\{\mathcal{H}_{y} \mid y \in Y\right\}$ of Hilbert spaces, and the family $\left\{s_{\alpha}\right\}_{\alpha \in A}$ of maps, is the vector space

$\mathcal{H}=\int_{Y} \mathcal{H}_{y} d \tau(y):$ all maps $s: Y \rightarrow \bigcup_{y \in Y} \mathcal{H}_{y}$ such that

(i) $s(y) \in \mathcal{H}_{y}$ a.e. $(Y, \tau)$,

(ii) $y \mapsto\left\langle s(y), s_{\alpha}(y)\right\rangle_{\mathcal{H}_{y}}$ is measurable, for each $\alpha \in A$, and

(iii) $y \mapsto\left\langle s(y), s_{\alpha}(y)\right\rangle_{\mathcal{H}_{y}}$ belongs to $L^{1}(Y, \tau)$, for all $\alpha \in A$

with inner product $\left\langle s, s^{\prime}\right\rangle=\int_{Y}\left\langle s(y), s^{\prime}(y)\right\rangle_{\mathcal{H}_{y}} d \tau(y)$.

The inner product of Definition 2.15 is well defined, and the direct integral $\mathcal{H}$ is a Hilbert space. Our problem now is to find an appropriate family $\left\{s_{\alpha}\right\}_{\alpha \in A}$ of maps to $\bigcup_{\pi_{n} \in \widehat{G}_{n}} \mathcal{A}\left(\pi_{n}\right)$ in order to put the $\mathcal{A}\left(\pi_{n}\right)$ together to make spaces $\mathcal{A}\left(G_{n, \chi}\right)$ and $\mathcal{A}\left(G_{n}\right)$ of regular functions on $G_{n}$ along the lines of a direct integral of Hilbert spaces. In other words we need the conditions that

$$
\begin{aligned}
& \text { to form } L^{2}\left(G_{n, \chi}\right) \\
& =\int_{G_{n, \chi}}^{\int}\left(\mathcal{H}_{\pi_{n}} \widehat{\otimes} \mathcal{H}_{\pi_{n}}^{*}\right) d_{n, \chi}\left(\pi_{n}\right) \text { we may choose the } s_{\alpha} \text { with } s_{\alpha}\left(\pi_{n}\right) \in \mathcal{A}\left(\pi_{n}\right)
\end{aligned}
$$

in order to form the $\mathcal{A}\left(G_{n, \chi}\right):=\int_{\widehat{G_{n, \chi}}} \mathcal{A}\left(\pi_{n}\right) d_{n, \chi}\left(\pi_{n}\right)$, and then we need the conditions

$$
\begin{aligned}
& \text { to form } L^{2}\left(G_{n}\right) \\
& =\int_{\widehat{Z}} L^{2}\left(G_{n, \chi}\right) d_{n}(\chi) \text { we may assume } s_{\alpha}\left(\frac{\int}{G_{n, \chi}}\left(\pi_{n} \otimes \pi_{n}^{*}\right) d_{n, \chi}\left(\pi_{n}\right)\right) \in \mathcal{A}\left(G_{n, \chi}\right) .
\end{aligned}
$$


These conditions are automatic when the $G_{n}$ are compact. In Sects. 4 and 5 we will verify them for the cases where $G_{n}$ is a connected simply connected nilpotent Lie group that has square integrable representations.

Under the assumptions (2.13) and (2.14) for forming our direct limits of Hilbert spaces, and (2.18) and (2.19) for restricting to regular functions before the direct limits, we have the spaces

$$
\mathcal{A}\left(G_{n}\right):=\int_{\widehat{Z}}\left(\int_{\widehat{G_{n, \chi}}} \mathcal{A}\left(\pi_{n}\right) d_{n, \chi}\left(\pi_{n}\right)\right) d(\chi) \text { and } \mathcal{A}(G):=\lim _{\longrightarrow} \mathcal{A}\left(G_{n}\right) .
$$

Here the $\mathcal{A}\left(G_{n}\right)$ form a direct system using ordinary restriction of functions, and $\mathcal{A}(G)$ is the direct limit of that system. Each $\mathcal{A}\left(G_{n}\right)$ is a dense subspace of $L^{2}\left(G_{n}\right)$ but, because of rescaling by the $\zeta_{m, n, \pi}$, we do not have $\mathcal{A}(G)$ as a subspace of $L^{2}(G)$. However there is a very useful relation which we now describe.

\subsection{Comparison of direct limits}

In this section we will assume (2.13), (2.14), (2.18) and (2.19). Then we have direct systems $\left\{L^{2}\left(G_{n}\right)\right\} \rightarrow L^{2}(G)$ and $\left\{\mathcal{A}\left(G_{n}\right)\right\} \rightarrow \mathcal{A}(G)$. Now define $\left(G_{n} \times G_{n}\right)$-equivariant isometric inclusions

$$
\eta_{n}: \mathcal{A}\left(\pi_{n}\right) \rightarrow \mathcal{H}_{\pi_{n}} \widehat{\otimes} \mathcal{H}_{\pi_{n}}^{*} \text { by } f_{u, v, n} \mapsto \sqrt{\operatorname{deg} \pi_{n}} f_{u, v, n}
$$

Proposition 2.22 The maps $\eta_{n}$ of (2.21) satisfy $\eta_{m} \circ \zeta_{m, n, \pi}\left(f_{u, v, n}\right)=\zeta_{m, n, \pi} \circ$ $\eta_{n}\left(f_{u, v, n}\right)$ and send the direct system $\left\{\mathcal{A}\left(G_{n}\right)\right\}$ into the direct system $\left\{L^{2}\left(G_{n}\right)\right\}$. That map of direct systems defines a $(G \times G)$-equivariant injection

$$
\eta: \mathcal{A}(G) \rightarrow L^{2}(G)
$$

with dense image. In particular $\eta$ defines a pre Hilbert space structure on $\mathcal{A}(G)$ with completion isometric to $L^{2}(G)$.

Proof We extract the result from [9, Appendix A]. Let $\mathcal{C}$ denote the category of topological vector spaces and continuous linear maps. We compute $\eta_{m} \circ \zeta_{m, n, \pi}\left(f_{u, v, n}\right)=$ $\eta_{n}\left(f_{u, v, n}\right)$, as asserted, and use (2.13) and (2.14) to patch the $\eta_{n}$ together to form maps $\widetilde{\eta_{n}}: \mathcal{A}\left(G_{n}\right) \rightarrow L^{2}\left(G_{n}\right)$. The space $L^{2}\left(G_{n}\right)$ carries its usual topology and we give $\mathcal{A}\left(G_{n}\right)$ the subspace topology. Now view $\left\{\mathcal{A}\left(G_{n}\right)\right\}$ and $\left\{L^{2}\left(G_{n}\right)\right\}$ as direct systems in the category $\mathcal{C}$. The $\widetilde{\eta_{n}}$ define a morphism $\left\{\mathcal{A}\left(G_{n}\right)\right\} \rightarrow\left\{L^{2}\left(G_{n}\right)\right\}$ of direct systems in $\mathcal{C}$. From the universal property of direct limits we then have a morphism $\tilde{\eta}: \lim _{\rightarrow \mathcal{C}}\left\{\mathcal{A}\left(G_{n}\right)\right\} \rightarrow \lim _{\rightarrow}\left\{L^{2}\left(G_{n}\right)\right\}$ of the direct limits in $\mathcal{C}$. Note that $\widetilde{\eta_{n}}$ is injective and $\left(G_{n} \times G_{n}\right)$-equivariant with dense image. It follows that $\widetilde{\eta}$ is injective and $(G \times G)$-equivariant with dense image.

The topological vector space direct limit $\lim _{\longrightarrow}\left\{L^{2}\left(G_{n}\right)\right\}$ has a pre Hilbert space structure given by the Hilbert space structures on the $L^{2}\left(G_{n}\right)$, and the Hilbert space 
direct limit $L^{2}(G)=\underset{\lim }{\longrightarrow}\left\{L^{2}\left(G_{n}\right)\right\}$ is its Hilbert space completion. Thus $\tilde{\eta}$ : $\lim _{\longrightarrow}\left\{\mathcal{C}\left(G_{n}\right)\right\} \rightarrow \lim _{\longrightarrow}\left\{\overrightarrow{L^{2}}\left(G_{n}\right)\right\}$ is in fact a continuous linear map, injective and $(G \times G)$-equivariant, from $\lim _{\longrightarrow}\left\{\mathcal{C}\left(G_{n}\right)\right\}$ to $L^{2}\left(G_{n}\right)$. Further, our original $\mathcal{A}(G)$ is obtained from $\lim _{\rightarrow}\left\{\mathcal{C}\left(G_{n}\right)\right\} \overrightarrow{\text { by }}$ forgetting the topology. Thus in particular $\tilde{\eta}$ maps $\mathcal{A}(G)$ into $L^{2}(G)$, and it is a $(G \times G)$-equivariant injection onto a dense subspace.

\subsection{Homogeneous spaces}

We now consider direct limit homogeneous spaces $G / K=\underline{\lim } G_{n} / K_{n}$. Specifically, we require that $G=\underline{\lim } G_{n}$, that the $K_{n}$ are compact subgroups of the $G_{n}$ such that $K_{n}=G_{n} \cap K_{m}$ for $\vec{m} \geqq n$, and that $K=\lim _{\longrightarrow} K_{n}$. We also assume (2.13), (2.14), (2.18) and (2.19) so that Proposition 2.22 applies to $\left\{G_{n}\right\}$ and $G$.

Since $K_{n}$ is compact, $L^{2}\left(G_{n} / K_{n}\right)=L^{2}\left(G_{n}\right)^{K_{n}}$, the subspace consisting of the right- $K_{n}$-invariant $L^{2}$ functions on $G_{n}$. Of course we can define $L^{2}(G / K)=L^{2}(G)^{K}$, but for comparison with $\mathcal{A}(G / K)$ we want $L^{2}(G / K)$ to be of the form $\lim _{\longrightarrow} L^{2}\left(G_{n} / K_{n}\right)$. Since $K_{n}$ is compact, (2.5) gives us

$$
L^{2}\left(G_{n} / K_{n}\right)=L^{2}\left(G_{n}\right)^{K_{n}}=\int_{\widehat{G_{n}}}\left(\mathcal{H}_{\pi_{n}} \widehat{\otimes}\left(\mathcal{H}_{\pi_{n}}^{*}\right)^{K_{n}}\right) d\left(\pi_{n}\right)
$$

we view the direct limit maps $\beta_{m, n, \pi}: \mathcal{H}_{\pi_{n}} \rightarrow \mathcal{H}_{\pi_{m}}$ as isometric inclusions and arrange the $L^{2}\left(G_{n} / K_{n}\right)$ into a direct system by requiring that

orthogonal projection $p_{m, n, \pi}: \mathcal{H}_{\pi_{m}} \rightarrow \mathcal{H}_{\pi_{n}}$ defines a bijective map $\mathcal{H}_{\pi_{m}}^{K_{m}} \rightarrow \mathcal{H}_{\pi_{n}}^{K_{n}}$.

Then every $u_{m} \in \mathcal{H}_{\pi_{m}}^{K_{m}}$ has unique expression $u_{m}=u_{n}+x$ with $u_{n} \in \mathcal{H}_{\pi_{n}}^{K_{n}}$ and $x \perp \beta_{m, n, \pi}\left(\mathcal{H}_{\pi_{n}}\right)$. As $\pi_{n}$ is irreducible and $\left\|u_{m}\right\|^{2}=\left\|u_{n}\right\|^{2}+\|x\|^{2}$ we have a constant $c=c_{m, n, \pi}$ such that $\left\|u_{n}\right\|=c\left\|u_{m}\right\|$. Here $0<c \leqq 1$ and $u_{n}=p_{m, n, \pi}\left(u_{m}\right)$. Thus we have an isometry

$$
\alpha_{m, n, \pi}: \mathcal{H}_{\pi_{n}}^{K_{n}} \cong \mathcal{H}_{\pi_{m}}^{K_{m}} \text { by } \alpha_{m, n, \pi}\left(u_{n}\right)=c_{m, n, \pi} u_{m} \text { where } u_{n}=p_{m, n, \pi}\left(u_{m}\right)
$$

Now we have $G_{n}$-equivariant isometric injections

$$
\begin{aligned}
\widetilde{\zeta}_{m, n, \pi}: \mathcal{H}_{\pi_{n}} \widehat{\otimes}\left(\mathcal{H}_{\pi_{n}}^{*}\right)^{K_{n}} & \rightarrow \mathcal{H}_{\pi_{m}} \widehat{\otimes}\left(\mathcal{H}_{\pi_{m}}^{*}\right)^{K_{m}} \text { defined by } f_{u, v_{n}, n} \\
& \mapsto c_{m, n, \pi}\left(\frac{\operatorname{deg} \pi_{m}}{\operatorname{deg} \pi_{n}}\right)^{1 / 2} f_{u, v_{m}, m} .
\end{aligned}
$$

Then $\left\{\left(\mathcal{H}_{\pi_{n}} \widehat{\otimes}\left(\mathcal{H}_{\pi_{n}}^{*}\right)^{K_{n}}\right), \widetilde{\zeta}_{m, n, \pi}\right\}$ is isomorphic to a subsystem of the Hilbert space direct system $\left\{\left(\mathcal{H}_{\pi_{n}} \widehat{\otimes} \mathcal{H}_{\pi_{n}}^{*}\right), \zeta_{m, n, \pi}\right\}$ of (2.12). As a result we have

$$
\mathcal{H}_{\pi} \widehat{\otimes}\left(\mathcal{H}_{\pi}^{*}\right)^{K}=\lim _{\longrightarrow}\left\{\left(\mathcal{H}_{\pi_{n}} \widehat{\otimes}\left(\mathcal{H}_{\pi_{n}}^{*}\right)^{K_{n}}\right), \widetilde{\zeta}_{m, n, \pi}\right\}
$$


in the Hilbert space category, and they fit together under the direct integral (2.23) to give us

$$
L^{2}(G / K):=L^{2}(G)^{K}=\lim _{\longrightarrow}\left\{L^{2}\left(G_{n} / K_{n}\right), \widetilde{\zeta}_{m, n}\right\}
$$

From (2.4) and (2.24) we have

$\mathcal{A}\left(\pi_{n}\right)^{K_{n}}=\left\{\right.$ finite linear combinations of the $f_{u, v_{n}, n}$ where $u \in \mathcal{H}_{\pi_{n}}$ and $\left.v_{n} \in \mathcal{H}_{\pi_{n}}^{K_{n}}\right\}$ and $v_{m, n, \pi}: \mathcal{A}\left(\pi_{n}\right)^{K_{n}} \hookrightarrow \mathcal{A}\left(\pi_{m}\right)^{K_{m}}$ by $f_{u, v_{n}, n} \mapsto f_{u, v_{m}, m}$ where projection $p_{m, n, \pi}\left(v_{m}\right)=v_{n}$.

Lemma 2.30 If $f \in \mathcal{A}\left(\pi_{n}\right)^{K_{n}}$ then $\left.v_{m, n, \pi}(f)\right|_{G_{n} / K_{n}}=f$.

Proof Let $u \in \mathcal{H}_{\pi_{n}}$ and $v_{n} \in \mathcal{H}_{\pi_{n}}^{K_{n}}$. Then $v_{m}=v_{n}+x \in \mathcal{H}_{\pi_{m}}^{K_{m}}$ with $x \perp \mathcal{H}_{\pi_{n}}$. Given $g \in G_{n}$ compute $\left(v_{m, n, \pi}\left(f_{u, v_{n}, n}\right)\right)(g)=f_{u, v_{m}, m}(g)=\left\langle u, \pi_{m}(g)\left(v_{n}+x\right)\right\rangle=$ $\left.\left\langle\pi_{m}\left(g^{-1}\right) u, v_{n}+x\right)\right\rangle=\left\langle\pi_{m}\left(g^{-1}\right) u, v_{n}\right\rangle=\left\langle u, \pi_{m}(g)\left(v_{n}\right)\right\rangle=\left\langle u, \pi_{n}(g)\left(v_{n}\right)\right\rangle=$ $f_{u, v_{n}, n}(g)$.

Equation (2.29) defines a direct system $\left\{\mathcal{A}\left(\pi_{n}\right)^{K_{n}}, v_{m, n, \pi}\right\}$ and Lemma 2.30 shows that its maps are inverse to restriction. Thus the direct limit

$$
\mathcal{A}(\pi)^{K}:=\lim _{\longrightarrow}\left\{\mathcal{A}\left(\pi_{n}\right)^{K_{n}}, v_{m, n, \pi}\right\}
$$

sits naturally as a $G$-submodule of the inverse $\operatorname{limit} \lim \left\{\mathcal{A}\left(\pi_{n}\right)^{K_{n}}\right.$, restriction $\}$. Now, from Proposition 2.22, we can take right $K$-invariants as follows.

Our assumptions (2.13) and (2.14) for forming our direct limits of Hilbert spaces, and (2.18) and (2.19) for restricting to regular functions before the direct limits, carry (2.20) over to $K_{n}$ - and $K$-invariant regular functions as follows.

$$
\begin{aligned}
\mathcal{A}\left(G_{n} / K_{n}\right) & :=\mathcal{A}\left(G_{n}\right)^{K_{n}}=\int_{\widehat{Z}}\left(\int_{G_{n, \chi}} \mathcal{A}\left(\pi_{n}\right)^{K_{n}} d_{n, \chi}\left(\pi_{n}\right)\right) d(\chi) \\
\text { and } \mathcal{A}(G / K) & :=\mathcal{A}(G)^{K}=\lim _{\longrightarrow}\left\{\mathcal{A}\left(G_{n} / K_{n}\right), v_{m, n}\right\} .
\end{aligned}
$$

In view of Lemma 2.30 the $\mathcal{A}\left(G_{n} / K_{n}\right)$ form a direct system whose maps are inverse to restriction of functions and $\mathcal{A}(G / K)$ is the direct limit of that system. Each $\mathcal{A}\left(G_{n} / K_{n}\right)$ is a dense subspace of $L^{2}\left(G_{n} / K_{n}\right)$ but, because the $v_{m, n}$ are not isometric, we do not have $\mathcal{A}(G / K)$ sitting naturally as a subspace of $L^{2}(G / K)$. As in the group level setting we can manage this in a somewhat abstract way. The $G_{n}$-equivariant isometric inclusions restrict to

$$
\tilde{\eta}_{n, \pi}: \mathcal{A}\left(\pi_{n}\right)^{K_{n}} \rightarrow \mathcal{H}_{\pi_{n}} \widehat{\otimes}\left(\mathcal{H}_{\pi_{n}}^{*}\right)^{K_{n}} \text { by } f_{u, v, n} \mapsto c_{n, 1, \pi} \sqrt{\operatorname{deg} \pi_{n}} f_{u, v, n}
$$


Proposition 2.34 The maps $\widetilde{\zeta}_{m, n, \pi}$ of (2.26), $v_{m, n, \pi}$ of (2.29) and $\widetilde{\eta}_{n, \pi}$ of (2.33) satisfy

$$
\left(\widetilde{\eta}_{m, \pi} \circ v_{m, n, \pi}\right)\left(f_{u, v, n}\right)=\left(\widetilde{\zeta}_{m, n, \pi} \circ \widetilde{\eta}_{n, \pi}\right)\left(f_{u, v, n}\right)
$$

for $f_{u, v, n} \in \mathcal{A}\left(\pi_{n}\right)^{K_{n}}$. Thus they inject the direct system $\left\{\mathcal{A}\left(G_{n}\right)^{K_{n}}, v_{m, n}\right\}$ into the direct system $\left\{L^{2}\left(G_{n}\right)^{K_{n}}, \widetilde{\zeta}_{m, n}\right\}$. That map of direct systems defines a $G$-equivariant injection

$$
\widetilde{\eta}: \mathcal{A}(G / K) \rightarrow L^{2}(G / K)
$$

with dense image. In particular $\tilde{\eta}$ defines a pre Hilbert space structure on $\mathcal{A}(G / K)$ with completion isometric to $L^{2}(G / K)$.

Proof The point is that $c_{m, n, \pi}=c_{m, m-1, \pi} \cdot c_{m-1, m-2, \pi} \cdot \ldots \cdot c_{n+1, n, \pi}$; so $c_{m, n, \pi}=$ $c_{m, 1, \pi} / c_{n, 1, \pi}$. Now compute

$$
\begin{aligned}
\left(\widetilde{\zeta}_{m, n, \pi} \circ \widetilde{\eta}_{n, \pi}\right)\left(f_{u, v_{n}, n}\right) & =\widetilde{\zeta}_{m, n, \pi}\left(c_{n, 1, \pi} \sqrt{\operatorname{deg} \pi_{n}} f_{u, v_{n}, n}\right) \\
& =c_{m, n, \pi}\left(\frac{\operatorname{deg} \pi_{m}}{\operatorname{deg} \pi_{n}}\right)^{1 / 2} c_{n, 1, \pi} \sqrt{\operatorname{deg} \pi_{n}} f_{u, v_{m}, m} \\
& =c_{m, 1, \pi} \sqrt{\operatorname{deg} \pi_{m}} f_{u, v_{m}, m}=\left(\widetilde{\eta}_{m, \pi} \circ v_{m, n, \pi}\right)\left(f_{u, v_{n}, n}\right) .
\end{aligned}
$$

Our assertions follow as in the proof of Proposition 2.22.

\subsection{Commutative spaces}

Suppose that the $G_{n} / K_{n}$ are commutative spaces, i.e. that the $\left(G_{n}, K_{n}\right)$ are Gelfand pairs. If $\pi_{n} \in \widehat{G_{n}}$ then $\operatorname{dim} \mathcal{H}_{\pi_{n}}^{K_{n}} \leqq 1$, in other words either $\mathcal{H}_{\pi_{n}}^{K_{n}}=0$ or there is a unique (up to scale) unit vector $v_{n} \in \mathcal{H}_{\pi_{n}}^{K_{n}}$. In the latter case (2.24) simply asserts that $v_{m}$ cannot be orthogonal to $\beta_{m, n, \pi}\left(v_{n}\right)$, so that Proposition 2.34 is valid. Here there is a problem: given $\mathcal{H}_{\pi_{n}}^{K_{n}} \neq 0$ and $m \geqq n$ we must have $\mathcal{H}_{\pi_{m}}^{K_{m}} \neq 0$. In the remainder of this paper we study two situations in which this holds: when the $G_{n} / K_{n}$ are compact symmetric spaces, and in many cases where the $G_{n} / K_{n}$ are commutative nilmanifolds.

\section{Limits of compact Lie groups and compact symmetric spaces}

Suppose that $G_{n}$ is a compact topological group. Then the Peter Weyl Theorem says that $L^{2}\left(G_{n}\right)$ is the Hilbert space direct sum of the spaces $\mathcal{A}\left(\pi_{n}\right)$ of coefficients, $\pi_{n} \in$ $\widehat{G_{n}}$. In particular one has a dense subspace of $L^{2}\left(G_{n}\right)$ given by the algebraic direct sum

$$
\mathcal{A}\left(G_{n}\right)=\sum_{\pi_{n} \in \widehat{G}_{n}} \mathcal{A}\left(\pi_{n}\right)
$$

When $G_{n}$ is a compact Lie group, the spectrum of the ring $\mathcal{A}\left(G_{n}\right)$ is a linear algebraic group, the associated algebraic group of $G_{n}$ for which $\mathcal{A}\left(G_{n}\right)$ is the ring of regular 
functions. This essentially is Tannaka's Theorem; see Chevalley [3, Chapter 6]. Thus $\mathcal{A}\left(G_{n}\right)$ is the ring of regular functions on $G_{n}$ as well as on its associated algebraic group. Note that the associated algebraic group is the complexification $\left(G_{n}\right)_{\mathbb{C}}$.

\subsection{Parabolic direct limits of groups}

We make the standing assumption for this section and the next that

$$
\begin{aligned}
& \text { the } G_{n} \text { are compact connected Lie groups and } \\
& \left\{G_{n}, \varphi_{m, n}\right\} \text { is a strict parabolic direct system. }
\end{aligned}
$$

Here parabolic means that the semisimple part of $\varphi_{m, n}\left(G_{n}\right)$ is the semisimple part of the centralizer of a toral subgroup of $G_{m}$, in other words that $\varphi_{m, n}\left(\left[G_{n}, G_{n}\right]\right) \mathbb{C}$ is the semisimple component of a parabolic subgroup of $\left(G_{m}\right)_{\mathbb{C}}$. Thus we have Cartan subalgebras $\mathfrak{h}_{n} \subset \mathfrak{g}_{n}$ such that $d \varphi_{m, n}\left(\mathfrak{h}_{n}\right) \subset \mathfrak{h}_{m}$ and simple root systems $\Psi_{n} \subset i \mathfrak{h}_{n}^{*}$ such that

$$
\text { if } \psi \in \Psi_{n} \text { then there is a unique } \psi^{\prime} \in \Psi_{m} \text { such that } d \varphi_{m, n}^{*}\left(\psi^{\prime}\right)=\psi \text {. }
$$

Now we can enumerate the simple root systems as

$$
\begin{aligned}
& \Psi_{n}=\left\{\psi_{n, 1}, \ldots, \psi_{n, \ell_{n}}\right\}, \ell_{n}=\operatorname{rank} \mathfrak{g}_{n}, \text { and } \\
& \quad \psi_{n, j}=d \varphi_{m, n}^{*}\left(\psi_{m, j}\right) \text { for } 1 \leqq j \leqq \ell_{n} \text { and } m \geqq n .
\end{aligned}
$$

Dually we have enumerations of the systems of fundamental weights as

$$
\begin{aligned}
& \Xi_{n}=\left\{\xi_{n, 1}, \ldots, \xi_{n, \ell_{n}}\right\}, \ell_{n}=\operatorname{rank} \mathfrak{g}_{n}, \text { and } \\
& \quad \xi_{n, j}=d \varphi_{m, n}^{*}\left(\xi_{m, j}\right) \text { for } 1 \leqq j \leqq \ell_{n} \text { and } m \geqq n .
\end{aligned}
$$

Now the correspondence

$$
k_{1} \xi_{n, 1}+\cdots+k_{\ell_{n}} \xi_{n, \ell_{n}} \mapsto k_{1} \xi_{m, 1}+\cdots+k_{\ell_{m}} \xi_{n, \ell_{n}}
$$

sends dominant integral weights to dominant integral weights. Fix an index $n_{0}$ and a dominant integral weight $\lambda_{n_{0}}$ for $\left(\mathfrak{g}_{n_{0}}, \mathfrak{h}_{n_{0}}, \Psi_{n_{0}}\right)$. For $n \geqq n_{0}$ we now have a dominant integral weight $\lambda_{n}$ for $\left(\mathfrak{g}_{n}, \mathfrak{h}_{n}, \Psi_{n}\right)$ and a corresponding direct system of irreducible representations of the $G_{n}$ given by

$$
\begin{aligned}
\left\{\pi_{n, \lambda}\right\} \text { where } \lambda_{n_{0}} & =k_{1} \xi_{n_{0}, 1}+\cdots+k_{\ell_{n_{0}}} \xi_{n_{0}, \ell_{n_{0}}} \text { and } \\
\pi_{n, \lambda} & \in \widehat{G_{n}} \text { has highest weight } \lambda_{n}=k_{1} \xi_{n, 1}+\cdots+k_{\ell_{n_{0}}} \xi_{n, \ell_{n_{0}}} .
\end{aligned}
$$

Choose unit highest weight vectors $v_{n, \lambda} \in \mathcal{H}_{\pi_{n, \lambda}}$. We embed representation spaces

$$
\beta_{m, n, \lambda}=\beta_{m, n, \pi_{\lambda}}: \mathcal{H}_{\pi_{n, \lambda}} \hookrightarrow \mathcal{H}_{\pi_{m, \lambda}} \text { by } X\left(v_{n, \lambda}\right) \mapsto X\left(v_{m, \lambda}\right) \quad \text { for } X \in \mathcal{U}\left(\mathfrak{g}_{n}\right) \text {. }
$$


Then we have the direct limit representation and its representation space:

$$
\pi_{\lambda}=\underline{\lim } \pi_{n, \lambda} \text { unitary representation of } G=\underline{\lim } G_{n} \text { on } \mathcal{H}_{\pi_{\lambda}}=\underline{\lim } \mathcal{H}_{\pi_{n, \lambda}} \text {. }
$$

As in (2.11) we now have isometric injections

$$
\zeta_{m, n}: \mathcal{H}_{\pi_{n, \lambda}} \widehat{\otimes} \mathcal{H}_{\pi_{n, \lambda}}^{*} \rightarrow \mathcal{H}_{\pi_{m, \lambda}} \widehat{\otimes} \mathcal{H}_{\pi_{m, \lambda}}^{*} \text { defined by } f_{u, v, n} \mapsto\left(\frac{\operatorname{deg} \pi_{m, \lambda}}{\operatorname{deg} \pi_{n, \lambda}}\right)^{1 / 2} f_{u, v, m}
$$

That gives us direct systems of Hilbert spaces and the direct limits

$$
\mathcal{H}_{\pi_{\lambda}} \widehat{\otimes} \mathcal{H}_{\pi_{\lambda}}^{*}=\underline{\lim }\left\{\left(\mathcal{H}_{\pi_{n, \lambda}} \widehat{\otimes} \mathcal{H}_{\pi_{n, \lambda}}^{*}\right), \zeta_{m, n}\right\}
$$

They are the representation spaces for the irreducible unitary representations $\pi_{\lambda}$ of $G$ and $\pi_{\lambda} \otimes \pi_{\lambda}^{*}$ of $G \times G$. The Peter-Weyl Theorem for Parabolic Direct Limits [18, Theorem 4.3] exhibits the Hilbert space $L^{2}(G):=\lim _{\longrightarrow}\left\{L^{2}\left(G_{n}\right), \zeta_{m, n}\right\}$ as the orthogonal direct sum of the $\mathcal{H}_{\pi_{\lambda}} \widehat{\otimes} \mathcal{H}_{\pi_{\lambda}}^{*}$, and shows that the left/right regular representation of $G \times G$ on $L^{2}(G)$ the multiplicity-free discrete direct sum of irreducible representations $\pi_{\lambda} \otimes \pi_{\lambda}^{*}$ of highest weights $\left(\lambda, \lambda^{*}\right)$.

The direct integral conditions (2.13) and (2.14) are automatic here because the integrals are direct sums. Thus, as $\lambda$ varies, the (3.9) and their limits (3.10) sum to give us

$\zeta_{m, n}: L^{2}\left(G_{n}\right) \rightarrow L^{2}\left(G_{m}\right)$ defined by $f_{u, v, n} \mapsto\left(\frac{\operatorname{deg} \pi_{m, \lambda}}{\operatorname{deg} \pi_{n, \lambda}}\right)^{1 / 2} f_{u, v, m} \quad$ for $u, v \in \mathcal{H}_{\pi_{n, \lambda}}$

and the limit Hilbert space

$$
L^{2}(G):=\lim _{\longrightarrow}\left\{L^{2}\left(G_{n}\right), \zeta_{m, n}\right\}
$$

\subsection{The ring of regular functions for parabolic direct limits}

As noted earlier, the direct integral conditions (2.13), (2.14), (2.18) and (2.19) are automatic in the compact group setting. Thus Proposition 2.22 applies, showing that the $\mathcal{A}\left(G_{n}\right)$ and $\mathcal{A}(G)$ follow the same pattern as in the Peter-Weyl Theorem for Parabolic Direct Limits, and the resulting map $\mathcal{A}(G) \rightarrow L^{2}(G)$ defines both a pre Hilbert space structure on $\mathcal{A}(G)$ and an interpretation of the elements of $L^{2}(G)$ as the Hilbert space completion of $\mathcal{A}(G)$.

\subsection{Parabolic direct limits of compact symmetric spaces}

Fix a parabolic direct system of compact connected Lie groups $G_{n}$ and subgroups $K_{n}$ such that each $\left(G_{n}, K_{n}\right)$ is an irreducible Riemannian symmetric pair. Suppose that the 
corresponding compact symmetric spaces $M_{n}=G_{n} / K_{n}$ are simply connected. Up to re-numbering and passage to a common cofinal subsequence the only possibilities are

\begin{tabular}{|c|l|l|c|c|}
\hline \multicolumn{5}{|c|}{ Compact Irreducible Riemannian Symmetric Spaces $M_{n}=G_{n} / K_{n}$} \\
\hline \hline \multicolumn{1}{|c|}{$G_{n}$} & \multicolumn{1}{|c|}{$K_{n}$} & $\operatorname{Rank} M_{n}$ & $\operatorname{Dim} M_{n}$ \\
\hline \hline 1 & $S U(n) \times S U(n)$ & diagonal $S U(n)$ & $n-1$ & $n^{2}-1$ \\
\hline 2 & $\operatorname{Spin}(2 n+1) \times \operatorname{Spin}(2 n+1)$ & diagonal $\operatorname{Spin}(2 n+1)$ & $n$ & $2 n^{2}+n$ \\
\hline 3 & $\operatorname{Spin}(2 n) \times \operatorname{Spin}(2 n)$ & diagonal $\operatorname{Spin}(2 n)$ & $n$ & $2 n^{2}-n$ \\
\hline 4 & $S p(n) \times \operatorname{Sp}(n)$ & diagonal $\operatorname{Sp}(n)$ & $n$ & $2 n^{2}+n$ \\
\hline 5 & $S U(p+q), p=p_{n}, q=q_{n}$ & $S(U(p) \times U(q))$ & $\min (p, q)$ & $2 p q$ \\
\hline 6 & $S U(n)$ & $S O(n)$ & $n-1$ & $\frac{(n-1)(n+2)}{2}$ \\
\hline 7 & $S U(2 n)$ & $S p(n)$ & $n-1$ & $2 n^{2}-n-1$ \\
\hline 8 & $S O(p+q), p=p_{n}, q=q_{n}$ & $S O(p) \times S O(q)$ & $\min (p, q)$ & $p q$ \\
\hline 9 & $S O(2 n)$ & $U(n)$ & {$\left[\frac{n}{2}\right]$} & $n(n-1)$ \\
\hline 10 & $S p(p+q), p=p_{n}, q=q_{n}$ & $S p(p) \times S p(q)$ & $\min (p, q)$ & $4 p q$ \\
\hline 11 & $S p(n)$ & $U(n)$ & $n$ & $n(n+1)$ \\
\hline
\end{tabular}

These are the cases where the $G_{n}$ form a parabolic direct system. Now we set about carrying the results of Sects. 3.1 and 3.2 from $G=\lim _{\longrightarrow} G_{n}$ to $M=\underline{\lim } M_{n}$ for the systems of Table 3.13 .

\subsection{Square integrable functions and regular functions}

Recall the decomposition $\mathfrak{g}_{n}=\mathfrak{k}_{n}+\mathfrak{s}_{n}$ under the symmetry $\theta$ of $M_{n}$. Here $K_{n}$ is the identity component of the fixed point set $G_{n}^{\theta}, \mathfrak{k}_{n}$ is the +1 eigenspace of $d \theta$, and $\mathfrak{s}_{n}$ is the -1 eigenspace. We assume the alignments $\theta_{n}=\left.\theta_{m}\right|_{G_{n}}$ so $\mathfrak{k}_{n} \subset \mathfrak{k}_{m}$ and $\mathfrak{s}_{n} \subset \mathfrak{s}_{m}$. We recursively choose maximal abelian subspaces $\mathfrak{a}_{n} \subset \mathfrak{s}_{n}$ with $\mathfrak{a}_{n} \subset \mathfrak{a}_{m}$ and define $\mathfrak{m}_{n}$ to be the centralizer of $\mathfrak{a}_{n}$ in $\mathfrak{k}_{n}$. For the systems of Table 3.13 we have $\mathfrak{m}_{n} \subset \mathfrak{m}_{m}$, and we recursively choose Cartan subalgebras $\mathfrak{t}_{n} \subset \mathfrak{m}_{n}$ with that $\mathfrak{t}_{n} \subset \mathfrak{t}_{m}$. Then the $\mathfrak{h}_{n}:=\mathfrak{t}_{n}+\mathfrak{a}_{n}$ are $\theta$-stable Cartan subalgebras of the $\mathfrak{g}_{n}$.

The restricted root systems

$$
\Delta\left(\mathfrak{g}_{n}, \mathfrak{a}_{n}\right)=\left\{\left.\alpha\right|_{\mathfrak{a}_{n}} \mid \alpha \in \Delta\left(\mathfrak{g}_{n}, \mathfrak{h}_{n}\right) \text { and }\left.\alpha\right|_{\mathfrak{a}_{n}} \neq 0\right\}
$$

have consistent root orders

$$
\text { if } \alpha \in \Delta^{+}\left(\mathfrak{g}_{m}, \mathfrak{a}_{m}\right) \quad \text { and }\left.\quad \alpha\right|_{\mathfrak{a}_{n}} \neq 0 \quad \text { then }\left.\alpha\right|_{\mathfrak{a}_{n}} \in \Delta^{+}\left(\mathfrak{g}_{n}, \mathfrak{a}_{n}\right) \text {, }
$$

and similarly we have consistent root orders on the $\Delta\left(\mathfrak{m}_{n}, \mathfrak{t}_{n}\right)$. Together they define consistent positive root systems

$$
\begin{aligned}
\Delta^{+}\left(\mathfrak{g}_{n}, \mathfrak{h}_{n}\right)= & \left\{\alpha \in \Delta\left(\mathfrak{g}_{n}, \mathfrak{h}_{n}\right) \mid \text { either }\left.\alpha\right|_{\mathfrak{a}_{n}} \in \Delta^{+}\left(\mathfrak{g}_{n}, \mathfrak{a}_{n}\right), \text { or }\left.\alpha\right|_{\mathfrak{a}_{n}}=0\right. \text { and } \\
& \left.\left.\alpha\right|_{\mathfrak{t}_{n}} \in \Delta^{+}\left(\mathfrak{m}_{n}, \mathfrak{t}_{n}\right)\right\} .
\end{aligned}
$$

Using the parabolic condition we have simple restricted root systems

$\Psi_{n}=\Psi\left(\mathfrak{g}_{n}, \mathfrak{a}_{n}\right)$ such that if $\psi \in \Psi_{n}$ then there is a unique $\psi^{\prime} \in \Psi_{m}$ such that $\psi=\left.\psi^{\prime}\right|_{\mathfrak{a}_{n}}$. 
Now as in (3.4) we enumerate

$$
\Psi_{n}=\left\{\psi_{n, 1}, \ldots, \psi_{n, \ell_{n}}\right\}, \ell_{n}=\operatorname{dim} \mathfrak{a}_{n} \text {, and } \psi_{n, j}=\left.\psi_{m, j}\right|_{\mathfrak{a}_{n}} \text { for } 1 \leqq j \leqq \ell_{n} \text { and } m \geqq n
$$

Using the root orders just described, the Cartan-Helgason Theorem says that the irreducible representation $\pi_{\lambda}$ of $\mathfrak{g}_{n}$ of highest weight $\lambda$ gives a summand of the representation of $G_{n}$ on $L^{2}\left(M_{n}\right)$ if and only if (i) $\left.\lambda\right|_{\mathfrak{t}_{n}}=0$, so we may view $\lambda$ as an element of $i \mathfrak{a}_{n}^{*}$, and (ii) if $\alpha \in \Delta^{+}\left(\mathfrak{g}_{n}, \mathfrak{a}_{n}\right)$ then $\frac{\langle\lambda, \alpha\rangle}{\langle\alpha, \alpha\rangle}$ is an integer $\geqq 0$.

Condition (i) of the Cartan-Helgason Theorem persists under restriction $\lambda \mapsto$ $\left.\lambda\right|_{\mathfrak{h}_{n-1}}$ because $\mathfrak{t}_{n-1} \subset \mathfrak{t}_{n}$. Given (i), condition (ii) says that $\frac{1}{2} \lambda$ belongs to the weight lattice of $\mathfrak{g}_{n}$, so its restriction to $\mathfrak{h}_{n-1}$ exponentiates to a well defined function on the corresponding maximal torus of $G_{n-1}$ and thus belongs to the weight lattice of $\mathfrak{g}_{n-1}$. Given condition (i), our arrangements $\mathfrak{a}_{n} \subset \mathfrak{a}_{m}$ and $\mathfrak{t}_{n} \subset \mathfrak{t}_{m}$ ensure that condition (ii) persists under restrictions $\left.\lambda \mapsto \lambda\right|_{\mathfrak{h}_{n-1}}$. With this in mind, we define linear functionals $\xi_{n, j} \in i \mathfrak{a}_{n}^{*}$ by

$$
\frac{\left\langle\xi_{n, i}, \psi_{n, j}\right\rangle}{\left\langle\psi_{n, j}, \psi_{n, j}\right\rangle}=\delta_{i, j} \quad \text { for } 1 \leqq j \leqq r_{n} \text {-except that } \frac{\left\langle\xi_{n, i}, \psi_{n, i}\right\rangle}{\left\langle\psi_{n, i}, \psi_{n, i}\right\rangle}=2 \quad \text { if } 2 \psi_{n, i} \in \Delta\left(\mathfrak{g}_{n}, \mathfrak{a}_{n}\right) .
$$

The weights $\xi_{n, j}$ are the class 1 fundamental highest weights for $\left(\mathfrak{g}_{n}, \mathfrak{k}_{n}\right)$ Now as in Sect. 3.1 the correspondence

$$
k_{1} \xi_{n, 1}+\cdots+k_{\ell_{n}} \xi_{n, \ell_{n}} \mapsto k_{1} \xi_{m, 1}+\cdots+k_{\ell_{m}} \xi_{n, \ell_{n}}
$$

sends class 1 dominant integral weights to class 1 dominant integral weights and defines direct systems of irreducible class 1 representations by

$$
\begin{aligned}
\left\{\pi_{n, \lambda}\right\} \text { where } \lambda_{n_{0}} & =k_{1} \xi_{n_{0}, 1}+\cdots+k_{\ell_{n_{0}}} \xi_{n_{0}, \ell_{n_{0}}} \text { and } \\
\pi_{n, \lambda} & \in \widehat{G_{n}} \text { has highest weight } \lambda_{n}=k_{1} \xi_{n, 1}+\cdots+k_{\ell_{n_{0}}} \xi_{n, \ell_{n_{0}}} .
\end{aligned}
$$

As before, the corresponding embeddings of representation spaces $\beta_{m, n, \lambda}: \mathcal{H}_{\pi_{n, \lambda}} \rightarrow$ $\mathcal{H}_{\pi_{n, \lambda}}$ are given by choices of highest weight unit vectors $v_{n, \lambda} \in \mathcal{H}_{\pi_{n, \lambda}}$ and by $X\left(v_{n, \lambda}\right) \mapsto X\left(v_{m, \lambda}\right)$ for $X \in \mathcal{U}\left(\mathfrak{g}_{n}\right)$. Again we have the direct limit representations and its representation space

$$
\pi_{\lambda}=\lim _{\longrightarrow} \pi_{n, \lambda} \text { unitary representation of } G=\lim _{\longrightarrow} G_{n} \text { on } \mathcal{H}_{\pi_{\lambda}}=\underset{\lim }{\longrightarrow}\left\{\mathcal{H}_{\pi_{n, \lambda}}, \beta_{m, n, \lambda}\right\}
$$

Note that $\pi_{\lambda}$ is irreducible, has highest weight $\lambda$, and has a highest weight unit vector $v_{\lambda}=\lim _{\longrightarrow} v_{n, \lambda}$ that is invariant under the action of $K=\lim _{\longrightarrow} K_{n}$. Given $\left\{\lambda_{n}\right\}$ as in (3.16) we write $\mathcal{H}_{n, \lambda}$ for $\mathcal{H}_{\pi_{n, \lambda}}$. 
Lemma 3.18 For $\left\{\lambda_{n}\right\}$ as in (3.16) and each $n \geqq n_{0}$ let $w_{n, \lambda}$ be a unit vector in $\mathcal{H}_{n, \lambda}^{K_{n}}$. Then orthogonal projection $p_{m, n, \lambda}: \mathcal{H}_{m, \lambda} \rightarrow \mathcal{H}_{n, \lambda}$ maps $w_{m, \lambda}$ to a nonzero multiple $c_{m, n, \lambda} w_{n, \lambda}$ of $w_{n, \lambda}$.

Proof By $G_{n}$-equivariance of the projection, $p_{m, n, \lambda}\left(w_{m, \lambda}\right)$ is $K_{n}$-invariant, hence is a multiple of $w_{n, \lambda}$. Suppose that the multiple is zero, i.e. that $w_{n, \lambda} \perp w_{m, \lambda}$. Then $w_{m, \lambda} \perp \mathcal{H}_{n, \lambda}$, in particular $w_{m, \lambda}$ is orthogonal to the highest weight $\lambda$ vector $v_{n, \lambda}$. As $v_{m, \lambda}=v_{n, \lambda}$, now $w_{m, \lambda} \perp v_{m, \lambda}$. But the argument of [7, Proposition 2.2] shows that $w_{m, \lambda}$ cannot be orthogonal to $v_{m, \lambda}$. We conclude $p_{m, n, \lambda}\left(w_{m, \lambda}\right)=c w_{n, \lambda}$ for some nonzero constant $c$.

Denote $\lambda^{*}=-w_{0}(\lambda)$ where $w_{0}$ is the longest element of the Weyl group. Then $\lambda^{*}$ is the highest weight of the dual $\pi_{n, \lambda}^{*}$, and $\mathcal{H}_{\pi_{n, \lambda}}^{*}=\mathcal{H}_{\pi_{n, \lambda} *}$. Given $\pi_{n, \lambda} \in \widehat{G_{n, K}}$ we have $\pi_{n, \lambda^{*}} \in \widehat{G_{n, K}}$ and we may suppose that its $K_{n}$-fixed unit vector $w_{\lambda *}$ is normalized so that the pairing $\left(w_{\lambda}, w_{\lambda^{*}}\right)=1$. Then the space of right- $K_{n}$-invariant matrix coefficient functions corresponding to $\pi_{n, \lambda}$ is (as a module for the left translation action of $G_{n}$ )

$$
\left(\mathcal{H}_{\pi_{n, \lambda}} \otimes \mathcal{H}_{\pi_{n, \lambda}}^{*}\right)^{K_{n}}=\mathcal{H}_{\pi_{n, \lambda}} \otimes w_{\lambda *} \mathbb{C}=\left\{f_{u, w_{\lambda}, n} \mid u \in \mathcal{H}_{\pi_{n, \lambda}}\right\} \cong \mathcal{H}_{\pi_{n, \lambda}}
$$

Lemma 3.18 ensures (2.24) in our setting, where the regular representations of the $G_{n}$ on the $L^{2}\left(M_{n}\right)$ are multiplicity free. Thus we have $G_{n}$-equivariant injections

$$
\alpha_{m, n, \lambda^{*}}: \mathcal{H}_{n, \lambda^{*}}^{K_{n}} \rightarrow \mathcal{H}_{m, \lambda^{*}}^{K_{m}}, \quad \mathcal{H}_{n, \lambda}^{K_{n}}=w_{n, \lambda^{*}} \mathbb{C} \text { and } \mathcal{H}_{m, \lambda}^{K_{n}}=w_{m, \lambda^{*}} \mathbb{C}
$$

as in (2.25), and

$$
\begin{aligned}
\widetilde{\zeta}_{m, n, \lambda}: \mathcal{H}_{n, \lambda} \otimes\left(w_{n, \lambda} \mathbb{C}\right) & \rightarrow \mathcal{H}_{m, \lambda} \otimes\left(w_{m, \lambda} * \mathbb{C}\right) \text { by } f_{u, w_{n}, n} \\
& \mapsto c_{m, n, \lambda}\left(\frac{\operatorname{deg} \pi_{m, \lambda}}{\operatorname{deg} \pi_{n, \lambda}}\right)^{1 / 2} f_{u, w_{m}, m}
\end{aligned}
$$

as in (2.26). Now as in Sect. $2.5,\left\{\mathcal{H}_{n, \lambda} \otimes\left(w_{n, \lambda} * \mathbb{C}\right), \widetilde{\zeta}_{m, n, \lambda}\right\}$ is isomorphic to a subsystem of the system $\left\{\left(\mathcal{H}_{n, \lambda} \widehat{\otimes} \mathcal{H}_{n, \lambda}^{*}\right), \zeta_{m, n, \lambda}\right\}$ of (3.11). As a result we have

$$
\mathcal{H}_{\lambda} \otimes w_{\lambda *} \mathbb{C}:=\lim _{\longrightarrow}\left\{\left(\mathcal{H}_{n, \lambda} \otimes w_{n, \lambda *} \mathbb{C}\right), \widetilde{\zeta}_{m, n, \lambda}\right\}
$$

and they fit together under the direct integral (2.23), which here is reduced to a discrete direct sum, to give us $L^{2}(M)$ where $M=\underline{\lim } M_{n}$ as follows.

$$
L^{2}(M):=L^{2}(G)^{K}=\lim _{\longrightarrow}\left\{L^{2}\left(G_{n} / K_{n}\right), \widetilde{\zeta}_{m, n}\right\}
$$

We proceed as in Sect. 2.5, but taking advantage of the fact that here the $G_{n}$ are compact. Define

$\mathcal{A}\left(\pi_{n, \lambda}\right)^{K_{n}}=\left\{\right.$ finite linear combinations of the $f_{u, v_{n}, n}$ where $u \in \mathcal{H}_{\pi_{n, \lambda}}$ and $\left.v_{n} \in \mathcal{H}_{\pi_{n, \lambda}}^{K_{n}}\right\}$, $v_{m, n, \lambda}: \mathcal{A}\left(\pi_{n, \lambda}\right)^{K_{n}} \hookrightarrow \mathcal{A}\left(\pi_{m, \lambda}\right)^{K_{m}}$ by $f_{u, v_{n}, n} \mapsto f_{u, v_{m}, m}$ where projection $p_{m, n, \lambda}\left(v_{m}\right)$

$$
=v_{n} \text {. }
$$


Thus Lemma 2.30 says: If $f \in \mathcal{A}\left(\pi_{n, \lambda}\right)^{K_{n}}$ then $\left.v_{m, n, \lambda}(f)\right|_{G_{n} / K_{n}}=f$.

The ring of regular functions on $M_{n}=G_{n} / K_{n}$ is $\mathcal{A}\left(G_{n} / K_{n}\right):=\mathcal{A}\left(G_{n}\right)^{K_{n}}=$ $\sum_{\text {is }} \mathcal{A}\left(\pi_{n, \lambda}\right)$ and the $v_{m, n, \lambda}$ sum to define a direct system $\left\{\mathcal{A}\left(G_{n} / K_{n}\right), v_{m, n}\right\}$. Its limit

$$
\mathcal{A}(G / K):=\mathcal{A}(G)^{K}=\lim _{\longrightarrow}\left\{\mathcal{A}\left(G_{n} / K_{n}\right), v_{m, n}\right\}
$$

As before, the maps of the direct system $\left\{\mathcal{A}\left(G_{n} / K_{n}\right), v_{m, n}\right\}$ are inverse to restriction of functions, so $\mathcal{A}(G / K)$ is a $G$-submodule of the inverse $\operatorname{limit} \underset{\lim }{\longleftarrow}\left\{\mathcal{A}\left(G_{n} / K_{n}\right)\right.$, restriction $\}$.

Each $\mathcal{A}\left(G_{n} / K_{n}\right)$ is a dense subspace of $L^{2}\left(G_{n} / K_{n}\right)$ but, because the $v_{m, n}$ distort the Hilbert space structure, $\mathcal{A}(G / K)$ does not sit naturally as a subspace of $L^{2}(G / K)$. Thus we use the $G_{n}$-equivariant maps

$$
\widetilde{\eta}_{n, \lambda}: \mathcal{A}\left(\pi_{n, \lambda}\right)^{K_{n}} \rightarrow \mathcal{H}_{\pi_{n}} \widehat{\otimes}\left(w_{n, \lambda} * \mathbb{C}\right) \text { by } f_{u, w_{n, \lambda}, n} \mapsto c_{n, 1, \lambda} \sqrt{\operatorname{deg} \pi_{n, \lambda}} f_{u, w_{n, \lambda}, n} .
$$

where $c_{m, n, \lambda}=\left\|p_{m, n, \lambda}\left(w_{m, \lambda}\right)\right\|$. Now Proposition 2.34 specializes to

Proposition 3.27 The maps $\widetilde{\zeta}_{m, n, \lambda}$ of (3.21), $v_{m, n, \lambda}$ of (3.24) and $\widetilde{\eta}_{n, \lambda}$ of (3.26) satisfy

$$
\left(\widetilde{\eta}_{m, \lambda} \circ v_{m, n, \lambda}\right)\left(f_{u, v, n}\right)=\left(\widetilde{\zeta}_{m, n, \lambda} \circ \widetilde{\eta}_{n, \lambda}\right)\left(f_{u, v, n}\right)
$$

for $f_{u, v, n} \in \mathcal{A}\left(\pi_{n, \lambda}\right)^{K_{n}}$. Thus they inject the direct system $\left\{\mathcal{A}\left(G_{n}\right)^{K_{n}}, v_{m, n}\right\}$ into the direct system $\left\{L^{2}\left(G_{n}\right)^{K_{n}}, \widetilde{\zeta}_{m, n}\right\}$. That map of direct systems defines a $G$-equivariant injection

$$
\tilde{\eta}: \mathcal{A}(G / K) \rightarrow L^{2}(G / K)
$$

with dense image. In particular $\tilde{\eta}$ defines a pre Hilbert space structure on $\mathcal{A}(G / K)$ with completion isometric to $L^{2}(G / K)$.

\section{Limits of Heisenberg commutative spaces}

We now turn to a class of commutative spaces $G_{n} / K_{n}$ on which a nilpotent subgroup of $G_{n}$ acts transitively. Then that nilpotent group must be the nilradical $N_{n}$ of $G_{n}$, the isotropy subgroup $K_{n}$ must be a compact group of automorphisms of $N_{n}$, and $G_{n}$ must be the semidirect product $N_{n} \rtimes K_{n}$. See [16, Chapter 13] for an exposition. These spaces usually are weakly symmetric, but they are not symmetric. Nevertheless they are accessible because $N_{n}$ is very similar in structure to the Heisenberg group, and the theory is modelled on the Heisenberg group case. Here in Sect. 4 we study the Heisenberg group case, and we examine more general commutative nilmanifolds in Sect. 5. 
4.1 Regular functions on Heisenberg groups

We first consider the case where $G_{n}$ is the Heisenberg group

$$
\begin{aligned}
H_{n} & =\operatorname{Im} \mathbb{C}+\mathbb{C}^{n} \text { with group composition }(z, w)\left(z^{\prime}, w^{\prime}\right) \\
& =\left(z+z^{\prime}+\operatorname{Im}\left\langle w, w^{\prime}\right\rangle, w+w^{\prime}\right) .
\end{aligned}
$$

There are two sorts of irreducible unitary representations. The ones that annihilate the center $Z=\operatorname{Im} \mathbb{C}$ of $H_{n}$ are the (1-dimensional) unitary characters on the vector group $H_{n} / Z \cong \mathbb{R}^{2 n}$. The ones that are nontrivial on $Z$, say $\pi_{n, t}$ with central character $\zeta_{t}(z, w)=e^{t z}$, are specified by the nonzero real number $t$ and are realized on the Fock space $\mathcal{H}_{n, t}$ of entire holomorphic functions $f: \mathbb{C}^{n} \rightarrow \mathbb{C}$ such that $\int_{\mathbb{C}^{m}}|f(w)|^{2} e^{-|t||w|^{2}} d w<\infty$ where $d w$ is Lebesgue measure. The representation is $\left[\pi_{t}(z, v) f\right](w)=e^{t z \pm t \operatorname{Im}(w-v / 2) \cdot v} f(w-v)$ where \pm is the sign of $t /|t|$.

For each real $t \neq 0$ the representation $\pi_{n, t}$ is square integrable (modulo the center of $H_{n}$ ) and has formal degree $|t|^{n}$.

For each multi-index $\mathbf{m}=\left(m_{1}, \ldots, m_{n}\right), m_{i} \geqq 0$, we have the monomial $w^{\mathbf{m}}=$ $w_{1}^{m_{1}} \ldots w_{n}^{m_{n}}$. For $t \neq 0$ there is a constant $c_{n, t}>0$ such that the $\mu_{\mathbf{m}}:=w^{\mathbf{m}} / \sqrt{\mathbf{m} !}:=$ $\left(w_{1}^{m_{1}} \ldots w_{n}^{m_{n}}\right) / \sqrt{\left(m_{1} ! \ldots m_{n} !\right)}$ satisfy

$$
\int \mu_{\mathbf{m}}(w) \overline{\mu_{\mathbf{m}^{\prime}}(w)} \exp \left(-|t||w|^{2}\right) d w=\left\{\begin{array}{l}
0 \text { for } \mathbf{m} \neq \mathbf{m}^{\prime} \\
1 / c_{n, t} \text { for } \mathbf{m}=\mathbf{m}
\end{array}\right.
$$

Now we normalize the inner product on $\mathcal{H}_{\pi_{n, t}}=\mathcal{H}_{n, t}$ by

$$
\left\langle f, f^{\prime}\right\rangle=c_{n, t} \int f(w) \overline{f^{\prime}(w)} \exp \left(-|t||w|^{2}\right) d w .
$$

Then the monomials $\mu_{\mathbf{m}}$ form a complete orthonormal set in $\mathcal{H}_{\pi_{n, t}}$.

In Definition 2.15 of direct integral now, take $(Y, \mathcal{M}, \tau)=\left(\mathbb{R} \backslash\{0\}, \mathcal{M},|t|^{n} d t\right)$ where $\mathcal{M}$ consists of the Borel sets. For each multiindex m define $s_{\mathbf{m}}: Y: \rightarrow \cup \mathcal{H}_{n, t}$ by $s_{\mathbf{m}}(t)=e^{-|t|} \mu_{m}$. Then $\left\langle s_{\mathbf{m}}(t), s_{\mathbf{m}^{\prime}}(t)\right\rangle_{\mathcal{H}_{n, t}}=e^{-2|t|} \delta_{\mathbf{m}, \mathbf{m}^{\prime}} \in L^{1}(Y, \tau)$. In fact

$$
\begin{aligned}
\int_{-\infty}^{\infty}\left\langle s_{\mathbf{m}}(t), s_{\mathbf{m}}(t)\right\rangle_{\mathcal{H}_{n, t}}|t|^{n} d t & =2 \int_{0}^{\infty} e^{-2 t} t^{n} d t=2^{-n} \int_{0}^{\infty} e^{-2 t} 2^{n+1} t^{n} d t \\
& =2^{-n} \Gamma(n+1)=n ! / 2^{n}
\end{aligned}
$$

Thus (2.16) is satisfied and we have the Hilbert space of (2.17):

$$
\mathcal{H}_{n}=\int_{-\infty}^{\infty} \mathcal{H}_{n, t}|t|^{n} d t \text { defined by }\left(\mathbb{R} \backslash\{0\}, \mathcal{M},|t|^{n} d t\right) \text { and the } s_{\mathbf{m}}(t)=e^{-|t|} \mu_{\mathbf{m}}
$$


Here $|t|^{n} d t$ is the Plancherel measure for the Heisenberg group $H_{n}$. Since $|t|^{n} d t$ and $d t$ are mutually absolutely continuous on $\mathbb{R} \backslash\{0\}$ we can reformulate (4.3) as $\mathcal{H}_{n}=\int_{-\infty}^{\infty} \mathcal{H}_{n, t} d t$, which is independent of $n$, for purposes of taking direct limits.

For multiindices $\mathbf{m}$ and $\mathbf{m}^{\prime}$ define $r_{\mathbf{m}, \mathbf{m}^{\prime}}: Y: \rightarrow \bigcup \mathcal{H}_{n, t} \widehat{\otimes} \mathcal{H}_{n, t}^{*}$ by $r_{\mathbf{m}, \mathbf{m}^{\prime}}(t)=$ $e^{-|t|} \mu_{\mathbf{m}} \otimes \overline{\mu_{\mathbf{m}^{\prime}}}$. The $\mu_{\mathbf{m}} \otimes \overline{\mu_{\mathbf{m}^{\prime}}}$ form a complete orthonormal set in $\mathcal{H}_{n, t} \widehat{\otimes} \mathcal{H}_{n, t}^{*}$ so $\left\langle r_{\mathbf{m}, \mathbf{m}^{\prime}}(t), r_{\mathbf{m}^{\prime \prime}, \mathbf{m}^{\prime \prime \prime}}(t)\right\rangle_{\mathcal{H}_{n, t}}=e^{-2|t|} \delta_{\mathbf{m}, \mathbf{m}^{\prime \prime}} \delta_{\mathbf{m}^{\prime}, \mathbf{m}^{\prime \prime \prime}}$, which is in $L^{1}(Y, \tau)$ because $\int_{-\infty}^{\infty}\left\langle r_{\mathbf{m}, \mathbf{m}^{\prime}}(t), r_{\mathbf{m}, \mathbf{m}^{\prime}}(t)\right\rangle_{\mathcal{H}_{n, t}}|t|^{n} d t=2 \int_{0}^{\infty} e^{-2 t} t^{n} d t=2^{-n} \Gamma(n+1)=n ! / 2^{n}$. Since the 1-dimensional representations in $\widehat{H_{n}}$ form a set of Plancherel measure zero, and $|t|^{n} d t$ is the restriction of Plancherel measure to $\left\{\pi_{n, t} \mid t \neq 0\right\}$ this gives us

$$
L^{2}\left(H_{n}\right)=\int_{-\infty}^{\infty}\left(\mathcal{H}_{n, t} \widehat{\otimes} \mathcal{H}_{n, t}^{*}\right)|t|^{n} d t
$$

where the direct integral is defined by $\left(\mathbb{R} \backslash\{0\}, \mathcal{M},|t|^{n} d t\right)$ and the $r_{\mathbf{m}, \mathbf{m}^{\prime}}(t)=$ $e^{-|t|} \mu_{\mathbf{m}} \otimes \overline{\mu_{\mathbf{m}^{\prime}}}$. Now we carry this over to rational functions.

Definition 4.5 For each real polynomial $p(t)$ define $p_{\mathbf{m}, \mathbf{m}^{\prime}}(t)=e^{-|t|} p(t) \mu_{\mathbf{m}} \otimes \overline{\mu_{\mathbf{m}^{\prime}}}$. Let $\mathcal{A}\left(H_{n}\right)$ denote the set of all finite linear combinations of the $p_{\mathbf{m}, \mathbf{m}^{\prime}}$ with multiplication $\left(p_{\mathbf{m}, \mathbf{m}^{\prime}}^{\prime} \times p_{\ell, \ell^{\prime}}^{\prime \prime}\right)(t)=\left[p^{\prime}(t) p^{\prime \prime}(t)\right]\left(\mu_{\mathbf{m}+\ell} \otimes \overline{\mu_{\mathbf{m}^{\prime}+\ell^{\prime}}}\right)$. The elements of $\mathcal{A}\left(H_{n}\right)$ are the regular functions on $H_{n}$ and $\mathcal{A}\left(H_{n}\right)$ is the ring of regular functions on $H_{n}$. Note that $\mathcal{A}\left(H_{n}\right)$ is isomorphic to the ring of polynomials on the complex extension $\mathbb{C}+\mathbb{C}^{n}$ of the underlying vector space structure of $H_{n}=\operatorname{Im} \mathbb{C}+\mathbb{C}^{n}$.

Lemma 4.6 The ring $\mathcal{A}\left(H_{n}\right)$ of regular functions on $H_{n}$ is a dense subspace of $L^{2}\left(H_{n}\right)$.

Proof The pointwise inner product $\left\langle p_{\mathbf{m}, \mathbf{m}^{\prime}}(t), r_{\mathbf{m}^{\prime \prime}, \mathbf{m}^{\prime \prime \prime}}(t)\right\rangle_{\mathcal{H}_{n, t}}=e^{-2|t|} p(t) \delta_{\mathbf{m}, \mathbf{m}^{\prime \prime}}$ $\delta_{\mathbf{m}^{\prime}, \mathbf{m}^{\prime \prime \prime}}$. Computing as before, $\int_{-\infty}^{\infty} e^{-2|t|}\left|t^{k}\right||t|^{n} d t=(k+n) ! / 2^{k+n}$ for integral $k \geqq 0$. If $p(t)=\sum_{0}^{d} p_{k} t^{k}$ this shows

$$
\begin{aligned}
\left\|\left\langle p_{\mathbf{m}, \mathbf{m}^{\prime}}(t), r_{\mathbf{m}, \mathbf{m}^{\prime}}(t)\right\rangle_{\mathcal{H}_{n, t}}\right\|_{L^{1}\left(\mathbb{R} \backslash\{0\}, \mathcal{M},|t|^{n} d t\right)} & =\int_{-\infty}^{\infty}\left|e^{-2|t|} p(t)\right||t|^{n} d t \\
& \leqq \sum_{0}^{d}\left|p_{k}\right|(k+n) ! / 2^{k+n}<\infty,
\end{aligned}
$$

so each $\left\langle p_{\mathbf{m}, \mathbf{m}^{\prime}}(t), r_{\mathbf{m}^{\prime \prime}, \mathbf{m}^{\prime \prime \prime}}(t)\right\rangle_{\mathcal{H}_{n, t}} \in L^{1}\left(\mathbb{R} \backslash\{0\}, \mathcal{M},|t|^{n} d t\right)$. This proves $\mathcal{A}\left(H_{n}\right) \subset$ $L^{2}\left(H_{n}\right)$.

Let $f \in L^{2}\left(H_{n}\right)$ orthogonal to $\mathcal{A}\left(H_{n}\right)$. Denote $f_{t}(w)=f(t, w)$. and $r_{\mathbf{m}, \mathbf{m}^{\prime}, t}=$ $r_{\mathbf{m}, \mathbf{m}^{\prime}}(t)$. By (4.4) and Fubini, $f_{t}$ is orthogonal to every $r_{\mathbf{m}, \mathbf{m}^{\prime}, t}$ a.e. $t \in \mathbb{R}$. For fixed $t$, the $r_{\mathbf{m}, \mathbf{m}^{\prime}, t}$ form a complete orthogonal set in $\mathcal{H}_{n, t} \widehat{\otimes} \mathcal{H}_{n, t}^{*}$. Thus $f_{t}=0$ a.e. $t \in \mathbb{R}$. Now $f=0$ in $L^{2}\left(H_{n}\right)$. We have proved that the subspace $\mathcal{A}\left(H_{n}\right)$ is dense in $L^{2}\left(H_{n}\right)$. 


\subsection{Functions on the infinite Heisenberg group}

Passage to the limit is easy for Heisenberg groups. The group inclusions are given by $(z, w) \mapsto(z, w)$, identity on the center $\operatorname{Im} \mathbb{C}$ and the usual $\mathbb{C}^{n} \rightarrow \mathbb{C}^{m}$ by $\left(w_{1}, \ldots, w_{n}\right) \mapsto\left(w_{1}, \ldots, w_{n}, 0, \ldots, 0\right)$ on its complement. Also, and this is a key point, the Hilbert space $\mathcal{H}_{n, t}$ sits naturally in $\mathcal{H}_{m, t}$ as the closed span of the monomials

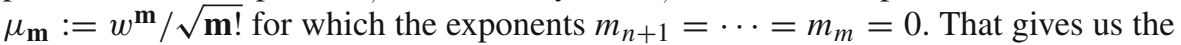
equivariant isometric inclusions $\zeta_{m, n}: \mathcal{H}_{n, t} \rightarrow \mathcal{H}_{m, t}$, sending $\mu_{\mathbf{m}}$ (as an element of $\mathcal{H}_{n, t}$ ) to $\mu_{\mathbf{m}}$ (as an element of $\mathcal{H}_{m, t}$ ). Now we have

$$
\begin{aligned}
H_{\infty} & =\operatorname{Im} \mathbb{C}+\mathbb{C}^{\infty}=\underline{\lim } H_{n}: \text { infinite Heisenberg group, } \\
\mathcal{H}_{t} & =\overleftrightarrow{\lim } \mathcal{H}_{n, t}: \text { Hilbert space with complete orthonormal set }\left\{\mu_{\mathbf{m}}=w^{\mathbf{m}} / \sqrt{\mathbf{m} !}\right\}, \\
\pi_{t} & =\overleftrightarrow{\longrightarrow} \pi_{n, t}: \text { irreducible unitary representation of } H_{\infty} \text { on } \mathcal{H}_{t} .
\end{aligned}
$$

The space $\mathcal{E}_{n, t}$ of matrix coefficients of $\mathcal{H}_{n, t}$ is spanned by the functions $f_{\ell, \mathbf{m} ; n, t}$ : $g \mapsto\left\langle\mu_{\ell}, \pi_{n, t}(g) \mu_{\mathbf{m}}\right\rangle$, These coefficients belong to the Hilbert space

$$
L^{2}\left(H_{n} / \operatorname{Im} \mathbb{C} ; e^{t}\right):=\left\{f: H_{n} \rightarrow \mathbb{C}|| f \mid \in L^{2}\left(H_{n} / \operatorname{Im} \mathbb{C}\right) \text { and } f(z, w)=e^{-t z} f(0, w)\right\}
$$

with inner product $\left\langle f, f^{\prime}\right\rangle=\int_{\mathbb{C}^{n}} f(z, w) \overline{f^{\prime}(z, w)} d w$.

Since $\pi_{n, t}$ has formal degree $|t|^{n}$ the orthogonality relations say that the inner product in $\mathcal{E}_{n, t}$ is $\left\langle f_{\ell, \mathbf{m} ; n, t}, f_{\ell^{\prime}, \mathbf{m}^{\prime} ; n, t}\right\rangle=|t|^{-n}$ if $\ell=\ell^{\prime}$ and $\mathbf{m}=\mathbf{m}^{\prime}, 0$ otherwise. Now the $|t|^{n / 2} f_{\ell, \mathbf{m} ; n, t}$ form a complete orthonormal set in $\mathcal{E}_{n, t}$, and $\mathcal{E}_{n, t}$ consists of the functions $\Phi_{n, t, \varphi}$ given by

$$
\Phi_{n, t, \varphi}(h)=\sum_{\ell, \mathbf{m}} \varphi_{\ell, \mathbf{m}}(t)|t|^{n / 2} f_{\ell, \mathbf{m} ; n, t}(h)
$$

where $\sum_{\ell, \mathbf{m}}\left|\varphi_{\ell, \mathbf{m}}(t)\right|^{2}<\infty$. Thus $L^{2}\left(H_{n}\right)$ consists of all functions

$$
\Psi_{n, \varphi}(h):=\int_{-\infty}^{\infty} \Phi_{n, t, \varphi}(h)|t|^{n} d t=\int_{-\infty}^{\infty}\left(\sum_{\ell, \mathbf{m}} \varphi_{\ell, \mathbf{m}}(t)|t|^{n / 2} f_{\ell, \mathbf{m} ; n, t}(h)\right)|t|^{n} d t
$$

such that the $\varphi_{\ell, \mathbf{m}}: \mathbb{R} \rightarrow \mathbb{C}$ are measurable, $\sum_{\ell, \mathbf{m}}\left|\varphi_{\ell, \mathbf{m}}(t)\right|^{2}<\infty$ for almost all $t \in \mathbb{R}$, and $\sum_{\ell, \mathbf{m}}\left|\varphi_{\ell, \mathbf{m}}(t)\right|^{2} \in L^{1}\left(\mathbb{R},|t|^{n} d t\right)$. Note that $\left\|\Psi_{n, \varphi}\right\|_{L^{2}\left(H_{n}\right)}^{2}=$ $\sum_{\ell, \mathbf{m}}\left\|\varphi_{\ell, \mathbf{m}}\right\|_{L^{2}\left(\mathbb{R},|t|^{n / 2} d t\right)}^{2}$.

When $m \geqq n$ we view an $n$-tuple $\mathbf{m}$ as an $m$-tuple by appending $m-n$ zeroes; then $w^{\mathbf{m}}$ has the same meaning as function on $\mathbb{C}^{n}$ and on $\mathbb{C}^{m}$. Thus the coefficient function $f_{\ell, \mathbf{m} ; n, t}: H_{n} \rightarrow \mathbb{C}$ is the restriction of $f_{\ell, \mathbf{m} ; n, t}: H_{m} \rightarrow \mathbb{C}$. Now, as in (2.11) and 
(3.9), we have isometric $\left(H_{n} \times H_{n}\right)$-equivariant injections

$$
\begin{aligned}
\zeta_{m, n, t}: \mathcal{E}_{n, t} \rightarrow \mathcal{E}_{m, t} \text { by } \zeta_{m, n, t}\left(|t|^{n / 2} f_{\ell, \mathbf{m} ; n, t}\right) & =|t|^{m / 2} f_{\ell, \mathbf{m} ; n, t} \text { and } \zeta_{m, n}\left(\Psi_{n, \varphi}\right) \\
& =\Psi_{m,|t|^{(n-m) / 2} \varphi}
\end{aligned}
$$

of $\mathcal{E}_{n, t}$ into $\mathcal{E}_{m, t}$ and $L^{2}\left(H_{n}\right)$ into $L^{2}\left(H_{m}\right)$. Specifically, $\zeta_{m, n, t}$ maps a complete orthonormal set in $\mathcal{E}_{n, t}$ to an orthonormal set in $\mathcal{E}_{m, t}$ and $\zeta_{m, n}$ passes to the direct integral. As expected, the result is the multiplicity-free left/right regular representation of $H_{\infty} \times H_{\infty}$ on

$$
L^{2}\left(H_{\infty}\right):=\lim _{\longrightarrow}\left\{L^{2}\left(H_{n}\right), \zeta_{m, n}\right\}
$$

Now look back to Definition 4.5, and define

$$
\begin{aligned}
\mathcal{A}\left(H_{\infty}\right) & =\underset{\rightarrow}{\lim } \mathcal{A}\left(H_{n}\right) \\
& =\left\{\text { finite linear combinations of the } p_{\mathbf{m}, \mathbf{m}^{\prime}}(t)=e^{-|t|} p(t) w^{\mathbf{m}} \overline{w^{\mathbf{m}^{\prime}}}\right\} .
\end{aligned}
$$

Thus $\mathcal{A}\left(H_{\infty}\right)$ consists of the finite linear combinations of the $e^{-|t|} t^{k} \mu_{\mathbf{m}} \overline{\mu_{\mathbf{m}^{\prime}}}$, and (for $n$ sufficiently large so that $\mu_{\mathbf{m}}, \mu_{\mathbf{m}^{\prime}} \in \mathcal{H}_{n, t}$ ) we have $\left\|e^{-|t|} t^{k} \mu_{\mathbf{m}} \overline{\mu_{\mathbf{m}^{\prime}}}\right\|_{L^{2}\left(H_{n}\right)}^{2}=$ $(k+n) ! / 2^{k+n}$. Consider the maps of (2.21):

$$
\begin{aligned}
\eta_{n, t}: \mathcal{A}\left(\pi_{n, t}\right) & \rightarrow \mathcal{H}_{n, t} \widehat{\otimes} \mathcal{H}_{n, t}^{*} \text { given by } \eta_{n, t}\left(e^{-|t|} p(t) \mu_{\mathbf{m}} \overline{\mu_{\mathbf{m}^{\prime}}}\right) \\
& =e^{\left(\left(\frac{n}{2}-1\right)|t|\right)} p(t) \mu_{\mathbf{m}} \overline{\mu_{\mathbf{m}^{\prime}}}
\end{aligned}
$$

Then Proposition 2.22 tells us that

Proposition 4.13 The maps $\eta_{m, t}$ satisfy $\eta_{m, t} \circ \zeta_{m, n, t}=\zeta_{m, n, t} \circ \eta_{n, t}$ on $\mathcal{A}\left(\pi_{n, t}\right)$ and send the direct system $\left\{\mathcal{A}\left(H_{n}\right)\right\}$ into the direct system $\left\{L^{2}\left(H_{n}\right)\right\}$. That direct system map defines an $\left(H_{\infty} \times H_{\infty}\right)$-equivariant injection $\eta: \mathcal{A}\left(H_{\infty}\right) \rightarrow L^{2}\left(H_{\infty}\right)$ with dense image. In particular $\eta$ defines a pre Hilbert space structure on $\mathcal{A}\left(H_{\infty}\right)$ with completion isometric to $L^{2}\left(H_{\infty}\right)$.

\subsection{Heisenberg nilmanifolds}

We first carry the results of Sects. 2.5 and 4.2 over to commutative spaces $G_{n} / K_{n}$ where $G_{n}$ is the semidirect product $H_{n} \rtimes K_{n}$ of a Heisenberg group with a compact group of automorphisms. The concrete results in this section will require that $K_{n}$ be connected and that its action on $\mathbb{C}^{n}$ be irreducible.

The classification goes as follows for the cases where $K_{n}$ is connected and is irreducible on $\mathbb{C}^{n}$. Carcano's Theorem ([2]; or see [1, Theorem 4.6] or [16, Theorem 13.2.2]) says that $\left(G_{n}, K_{n}\right)$ is a Gelfand pair if and only if the representation of 
$\left(K_{n}\right)_{\mathbb{C}}$, on polynomials on $\mathbb{C}^{n}$, is multiplicity free. Those groups were classified by Kač [5, Theorem 3] in another context. His list (as formulated in [16, (13.2.5)]) is

\begin{tabular}{|c|c|c|c|c|}
\hline \multicolumn{5}{|c|}{ Irreducible connected groups $K_{n} \subset U(n)$ multiplicity free on polynomials on $\mathbb{C}^{n}$} \\
\hline & Group $K_{n}$ & Group $\left(K_{n}\right)_{\mathbb{C}}$ & Acting on & Conditions on $n$ \\
\hline 1 & $S U(n)$ & $S L(n ; \mathbb{C})$ & $\mathbb{C}^{n}$ & $n \geqq 2$ \\
\hline 2 & $U(n)$ & $G L(n ; \mathbb{C})$ & $\mathbb{C}^{n}$ & $n \geqq 1$ \\
\hline 3 & $S p(m)$ & $S p(m ; \mathbb{C})$ & $\mathbb{C}^{n}$ & $n=2 m$ \\
\hline 4 & $U(1) \times S p(m)$ & $\mathbb{C}^{*} \times S p(m ; \mathbb{C})$ & $\mathbb{C}^{n}$ & $n=2 m$ \\
\hline 5 & $U(1) \times S O(n)$ & $\mathbb{C}^{*} \times S O(n ; \mathbb{C})$ & $\mathbb{C}^{n}$ & $n \geqq 2$ \\
\hline 6 & $U(m)$ & $G L(m ; \mathbb{C})$ & $S^{2}\left(\mathbb{C}^{m}\right)$ & $m \geqq 2, n=\frac{1}{2} m(m+1)$ \\
\hline 7 & $S U(m)$ & $S L(m ; \mathbb{C})$ & $\Lambda^{2}\left(\mathbb{C}^{m}\right)$ & $m$ odd, $n=\frac{1}{2} m(m-1)$ \\
\hline 8 & $U(m)$ & $G L(m ; \mathbb{C})$ & $\Lambda^{2}\left(\mathbb{C}^{m}\right)$ & $n=\frac{1}{2} m(m-1)$ \\
\hline 9 & $S U(\ell) \times S U(m)$ & $S L(\ell ; \mathbb{C}) \times S L(m ; \mathbb{C})$ & $\mathbb{C}^{\ell} \otimes \mathbb{C}^{m}$ & $n=\ell m, \ell \neq m$ \\
\hline 10 & $U(\ell) \times S U(m)$ & $G L(\ell ; \mathbb{C}) \times S L(m ; \mathbb{C})$ & $\mathbb{C}^{\ell} \otimes \mathbb{C}^{m}$ & $n=\ell m$ \\
\hline 11 & $U(2) \times S p(m)$ & $G L(2 ; \mathbb{C}) \times S p(m ; \mathbb{C})$ & $\mathbb{C}^{2} \otimes \mathbb{C}^{2 m}$ & $n=4 m$ \\
\hline 12 & $S U(3) \times S p(m)$ & $S L(3 ; \mathbb{C}) \times S p(m ; \mathbb{C})$ & $\mathbb{C}^{3} \otimes \mathbb{C}^{2 m}$ & $n=6 m$ \\
\hline 13 & $U(3) \times S p(m)$ & $G L(3 ; \mathbb{C}) \times S p(m ; \mathbb{C})$ & $\mathbb{C}^{3} \otimes \mathbb{C}^{2 m}$ & $n=6 m$ \\
\hline 14 & $U(4) \times S p(4)$ & $G L(4 ; \mathbb{C}) \times S p(4 ; \mathbb{C})$ & $\mathbb{C}^{4} \otimes \mathbb{C}^{8}$ & $n=32$ \\
\hline 15 & $S U(m) \times S p(4)$ & $S L(m ; \mathbb{C}) \times S p(4 ; \mathbb{C})$ & $\mathbb{C}^{m} \otimes \mathbb{C}^{8}$ & $n=8 m, m \geqq 3$ \\
\hline 16 & $U(m) \times S p(4)$ & $G L(m ; \mathbb{C}) \times S p(4 ; \mathbb{C})$ & $\mathbb{C}^{m} \otimes \mathbb{C}^{8}$ & $n=8 m, m \geqq 3$ \\
\hline 17 & $U(1) \times \operatorname{Spin}(7)$ & $\mathbb{C}^{*} \times \operatorname{Spin}(7 ; \mathbb{C})$ & $\mathbb{C}^{8}$ & $n=8$ \\
\hline 18 & $U(1) \times \operatorname{Spin}(9)$ & $\mathbb{C}^{*} \times \operatorname{Spin}(9 ; \mathbb{C})$ & $\mathbb{C}^{16}$ & $n=16$ \\
\hline 19 & $\operatorname{Spin}(10)$ & $\operatorname{Spin}(10 ; \mathbb{C})$ & $\mathbb{C}^{16}$ & $n=16$ \\
\hline 20 & $U(1) \times \operatorname{Spin}(10)$ & $\mathbb{C}^{*} \times \operatorname{Spin}(10 ; \mathbb{C})$ & $\mathbb{C}^{16}$ & $n=16$ \\
\hline 21 & $U(1) \times G_{2}$ & $\mathbb{C}^{*} \times G_{2, \mathbb{C}}$ & $\mathbb{C}^{7}$ & $n=7$ \\
\hline 22 & $U(1) \times E_{6}$ & $\mathbb{C}^{*} \times E_{6, \mathbb{C}}$ & $\mathbb{C}^{27}$ & $n=27$ \\
\hline
\end{tabular}

Now we have the direct systems

\begin{tabular}{|l|l|l|l|}
\hline \multicolumn{4}{|c|}{$\begin{array}{c}\text { Direct systems }\left\{\left(H_{n} \rtimes K_{n}, K_{n}\right)\right\} \text { of Gelfand pairs, } \\
K_{n} \text { connected and irreducible on } \mathbb{C}^{n}\end{array}$} \\
\hline & Group $K_{n}$ & Acting on & Conditions on $n$ \\
\hline 1 & $S U(n)$ & $\mathbb{C}^{n}$ & $n \geqq 2$ \\
\hline 2 & $U(n)$ & $\mathbb{C}^{n}$ & $n \geqq 1$ \\
\hline 3 & $S p(m)$ & $\mathbb{C}^{n}$ & $n=2 m$ \\
\hline 4 & $U(1) \times S p(m)$ & $\mathbb{C}^{n}$ & $n=2 m$ \\
\hline $5 \mathrm{a}$ & $U(1) \times S O(2 m)$ & $\mathbb{C}^{2 m}$ & $n=2 m \geqq 2$ \\
\hline $5 \mathrm{~b}$ & $U(1) \times S O(2 m+1)$ & $\mathbb{C}^{2 m+1}$ & $n=2 m+1 \geqq 3$ \\
\hline 6 & $U(m)$ & $S^{2}\left(\mathbb{C}^{m}\right)$ & $m \geqq 2, n=\frac{1}{2} m(m+1)$ \\
\hline 7 & $S U(m)$ & $\Lambda^{2}\left(\mathbb{C}^{m}\right)$ & $m$ odd, $n=\frac{1}{2} m(m-1)$ \\
\hline 8 & $U(m)$ & $\Lambda^{2}\left(\mathbb{C}^{m}\right)$ & $n=\frac{1}{2} m(m-1)$ \\
\hline 9 & $S U(\ell) \times S U(m)$ & $\mathbb{C}^{\ell} \otimes \mathbb{C}^{m}$ & $n=\ell m, \ell \neq m$ \\
\hline 10 & $S(U(\ell) \times U(m))$ & $\mathbb{C}^{\ell} \otimes \mathbb{C}^{m}$ & $n=\ell m$ \\
\hline 11 & $U(2) \times S p(m)$ & $\mathbb{C}^{2} \otimes \mathbb{C}^{2 m}$ & $n=4 m$ \\
\hline 12 & $S U(3) \times S p(m)$ & $\mathbb{C}^{3} \otimes \mathbb{C}^{2 m}$ & $n=6 m$ \\
\hline 13 & $U(3) \times S p(m)$ & $\mathbb{C}^{3} \otimes \mathbb{C}^{2 m}$ & $n=6 m$ \\
\hline 15 & $S U(m) \times S p(4)$ & $\mathbb{C}^{m} \otimes \mathbb{C}^{8}$ & $n=8 m, m \geqq 3$ \\
\hline 16 & $U(m) \times S p(4)$ & $\mathbb{C}^{m} \otimes \mathbb{C}^{8}$ & $n=8 m, m \geqq 3$ \\
\hline
\end{tabular}


In each case the direct system $\left\{K_{n}\right\}$ is both strict and parabolic. (We separated entry 5 of Table 4.14 into entries $5 \mathrm{a}$ and $5 \mathrm{~b}$ of Table 4.15 in order to have the parabolic property.)

We now suppose that $\left\{K_{n}\right\}$ is one of the strict parabolic direct system given by the rows of Table 4.15 .

As $U(n)$ acts on $H_{n}=\operatorname{Im} \mathbb{C}+\mathbb{C}^{n}$ by $k:(z, v) \mapsto(z, k v)$ it preserves the equivalence class of each of the square integrable representations $\pi_{n, t}$ of $H_{n}$. The Mackey obstruction vanishes and $\pi_{n, t}$ extends to a unitary representation $\widetilde{\pi_{n, t}}$ of $H_{n} \rtimes U(n)$ on $\mathcal{H}_{n, t}$. See [14, Section 4] for a geometric proof. We will also write $\widetilde{\pi_{n, t}}$ for its restriction, the extension of $\pi_{n, t}$ to a unitary representation of $G_{n}=H_{n} \rtimes K_{n}$.

Let $\kappa_{n, \lambda}$ denote the irreducible representation of $K_{n}$ of highest weight $\lambda$, and $\widetilde{\kappa_{n, \lambda}}$ its extension to $G_{n}=H_{n} \rtimes K_{n}$ which annihilates $H_{n}$. The corresponding representation space $\mathcal{F}_{n, \lambda}$ is a finite dimensional Hilbert space. Denote $\pi_{n, t, \lambda}=\widetilde{\pi_{n, t}} \otimes \widetilde{\kappa_{n, \lambda}}$. Then $\mathcal{H}_{n, t, \lambda}:=\mathcal{H}_{n, t} \otimes \mathcal{F}_{n, \lambda}$ is its representation space. Fix an orthonormal basis $\left\{u_{i}\right\}$ of $\mathcal{F}_{n, \lambda}$. Then $\left\{\mu_{\mathbf{m}} \otimes u_{i}\right\}$ is a complete orthonormal set in $\mathcal{H}_{n, t, \lambda}$, and we have matrix coefficients

$$
f_{\ell, \mathbf{m}, i, j ; n, t, \lambda}(h, k)=\left\langle\left(\mu_{\ell} \otimes u_{i}\right),\left(\left(\widetilde{\pi_{n, t}} \otimes \widetilde{\kappa_{n, \lambda}}\right)(h, k)\right)\left(\mu_{\mathbf{m}} \otimes u_{j}\right)\right\rangle .
$$

The formal degree $\operatorname{deg} \pi_{n, t, \lambda}=|t|^{n} \operatorname{deg}\left(\kappa_{n, \lambda}\right)$, so the $|t|^{n / 2} \operatorname{deg}\left(\kappa_{n, \lambda}\right)^{1 / 2} f_{\ell, \mathbf{m}, i, j ; n, t, \lambda}$ form a complete orthonormal set in the space $\mathcal{E}_{n, t, \lambda}=\mathcal{H}_{n, t, \lambda} \widehat{\otimes} \mathcal{H}_{n, t, \lambda}^{*}$ of matrix coefficient functions. As in (2.11), (3.9) and (4.10) we have isometric $\left(G_{n} \times G_{n}\right)$-equivariant injections

$$
\begin{aligned}
\zeta_{m, n, t, \lambda}: \mathcal{E}_{n, t, \lambda} & \rightarrow \mathcal{E}_{m, t, \lambda} \text { by } \zeta_{m, n, t, \lambda}\left(\left(|t|^{n} \operatorname{deg} \kappa_{n, \lambda}\right)^{1 / 2} f_{\ell, \mathbf{m}, i, j ; n, t, \lambda}\right) \\
& =\left(|t|^{m} \operatorname{deg} \kappa_{m, \lambda}\right)^{1 / 2} f_{\ell, \mathbf{m}, i, j ; m, t, \lambda}
\end{aligned}
$$

Integrate with respect to $t$ and sum on $\lambda$ to construct isometric $\left(G_{n} \times G_{n}\right)$-equivariant injections $\zeta_{m, n}: L^{2}\left(G_{n}\right) \rightarrow L^{2}\left(G_{m}\right)$.

Theorem 4.17 For $n>0$ let $K_{n}$ be a compact connected subgroup of $\operatorname{Aut}\left(H_{n}\right)$ such that $\left\{K_{n}\right\}$ is a strict parabolic direct system. Define $G_{n}=H_{n} \rtimes K_{n}, G=\underline{\lim }\left\{G_{n}\right\}$ and $K=\lim _{\longrightarrow}\left\{K_{n}\right\}$. Note $G=H_{\infty} \rtimes K$. Then $\left\{L^{2}\left(G_{n}\right), \zeta_{m, n}\right\}$ is a strict direct system of Hilbert spaces in which the maps $\zeta_{m, n}: L^{2}\left(G_{n}\right) \rightarrow L^{2}\left(G_{m}\right)$ are $\left(G_{n} \times G_{n}\right)$-equivariant unitary injections. That gives us the left/right regular representation of $G \times G$ on the Hilbert space $L^{2}(G):=\lim _{\longrightarrow}\left\{L^{2}\left(G_{n}\right), \zeta_{m, n}\right\}$. Further, that left/right regular representation is the multiplicity-free $\int_{-\infty}^{\infty}\left(\pi_{t, \lambda} \otimes \pi_{t, \lambda}^{*}\right) d t$ where $\pi_{t, \lambda}:=\underline{\lim } \pi_{n, t, \lambda}$.

Now the construction of $\mathcal{A}(G)$ follows the lines of (4.12), with properties relative to $L^{2}(G)$ as in Proposition 4.13. As in Definition 4.5 we define

$$
\begin{aligned}
\mathcal{A}(G) & =\underset{\longrightarrow}{\lim } \mathcal{A}\left(G_{n}\right) \text { where } \mathcal{A}\left(G_{n}\right) \\
& =\left\{\text { finite linear combinations of the } e^{-|t|} p(t) f_{\ell, \mathbf{m}, i, j ; n, t, \lambda}\right\}
\end{aligned}
$$


where $p(t)$ is a real polynomial in $t$. The argument of Lemma 4.6 shows that $\mathcal{A}\left(G_{n}\right)$ is a dense subspace of $L^{2}\left(G_{n}\right)$. The maps of (2.21) in this setting are

$$
\begin{aligned}
\eta_{n, t, \lambda}: \mathcal{A}\left(\pi_{n, t, \lambda}\right) & \rightarrow \mathcal{H}_{n, t, \lambda} \widehat{\otimes} \mathcal{H}_{n, t, \lambda}^{*} \text { by } \eta_{n, t, \lambda}\left(e^{-|t|} p(t) f_{\ell, \mathbf{m}, i, j ; n, t, \lambda}\right) \\
& =e^{\left(\frac{n}{2}-1\right)|t|} \sqrt{\operatorname{deg} \kappa_{n, \lambda}} p(t) f_{\ell, \mathbf{m}, i, j ; n, t, \lambda} .
\end{aligned}
$$

Then Proposition 2.22 tells us that

Proposition 4.19 The maps $\eta_{m, t, \lambda}$ satisfy $\eta_{m, t, \lambda} \circ \zeta_{m, n, t, \lambda}=\zeta_{m, n, t, \lambda} \circ \eta_{n, t, \lambda}$ on $\mathcal{A}\left(\pi_{n, t, \lambda}\right)$ and send the direct system $\left\{\mathcal{A}\left(G_{n}\right)\right\}$ into the direct system $\left\{L^{2}\left(G_{n}\right)\right\}$. That system map defines an $\left(\left(H_{\infty} \rtimes K\right) \times\left(H_{\infty} \rtimes K\right)\right)$-equivariant injection $\eta: \mathcal{A}\left(H_{\infty} \rtimes\right.$ $K) \rightarrow L^{2}\left(H_{\infty} \rtimes K\right)$ with dense image. In particular $\eta$ defines a pre Hilbert space structure on $\mathcal{A}\left(H_{\infty} \rtimes K\right)$ with completion isometric to $L^{2}\left(H_{\infty} \rtimes K\right)$.

Recall our working hypothesis that $\left\{K_{n}\right\}$ is one of the 16 systems of Table 4.15. Since $\left(G_{n}, K_{n}\right)$ is a Gelfand pair with $K_{n}$ irreducible on $\mathbb{C}^{n}$, Carcano's Theorem [2] says that the action of $K_{n}$ on the polynomial ring $\mathbb{C}\left[C^{n}\right]$ is multiplicity free, and it picks out the right $K_{n}$-invariants in $L^{2}\left(G_{n}\right)$, as follows.

Lemma 4.20 Recall the notation of Sect. 3.1 for $\Xi_{n}$, $\lambda$ and $\kappa_{n, \lambda} \in \widehat{K_{n}}$. Define $\widetilde{\kappa_{n, \lambda}} \in$ $\widehat{G_{n}}$ by $\widetilde{\kappa_{n, \lambda}}(h k)=\kappa_{n, \lambda}(k)$ for $h \in H_{n}$ and $k \in K_{n}$. Then $\pi_{n, t, \lambda}:=\widetilde{\pi_{n, t}} \otimes \widetilde{\kappa_{n, \lambda}}$ has a nonzero $K_{n}$-fixed vector if and only if $\kappa_{n, \lambda}^{*}$ occurs as a subrepresentation of $\widetilde{\pi_{n, t} \mid} K_{n}$, and in that case the space of $K_{n}$-fixed vectors has dimension 1.

Proof This is essentially the argument in [14, Section 14.5A]. Decompose $\widetilde{\pi_{n, t} \mid} K_{n}=$ $\sum_{\gamma \in \widetilde{K_{n}}} m_{\gamma} \gamma$. Carcano's Theorem ([2], or see [16, Theorem 13.2.2]) says that each $m_{\gamma}$ is either 0 or 1 . The $K_{n}$-fixed vectors of $\widetilde{\kappa} \otimes \widetilde{\pi_{n, t}}$ all occur in $\kappa \otimes\left(m_{\kappa^{*}} \kappa^{*}\right)$, and they form a space of dimension $m_{\kappa^{*}}$. The assertion follows.

We view $L^{2}\left(G_{n} / K_{n}\right)$ as the space of right- $K_{n}$-invariant functions in $L^{2}\left(G_{n}\right)$. With Lemma 4.20 in mind we set

$$
{\widehat{K_{n}}}^{\dagger}=\left\{\kappa_{n, \lambda} \in \widehat{K_{n}} \mid \kappa_{n, \lambda}^{*} \text { occurs in the space of polynomials on } \mathbb{C}^{n}\right\} \text {. }
$$

Recall that $\mathcal{F}_{n, \lambda}$ denotes the representation space of $\kappa_{n, \lambda}$. Given $\kappa_{n, \lambda} \in \widehat{K}_{n}^{\dagger}$ the right $K_{n}$-invariant in $\mathbb{C}\left[\mathbb{C}^{n}\right] \otimes \mathcal{F}_{n, \lambda}^{*}$ is $\int_{K_{n}} \sum_{i}\left(b_{i} \otimes \kappa_{n, \lambda}^{*}\left(b_{i}^{*}\right)\right) d k$ where $\left\{b_{i}\right\}$ is a basis of the $\kappa_{n, \lambda}$-subspace of $\mathbb{C}\left[\mathbb{C}^{n}\right]$ and $\left\{b_{i}^{*}\right\}$ is the dual basis of $\mathcal{F}_{n, \lambda}^{*}$. Normalize it to a unit vector $w_{n, t, \lambda}$. Then the (left regular) representation of $G_{n}$ on $L^{2}\left(G_{n} / K_{n}\right)$ is equivalent to the representation $\sum_{\kappa_{n, \lambda} \in \widehat{K}_{n}^{\dagger}} \int_{-\infty}^{\infty} \widetilde{\pi_{n, t}} \otimes \widetilde{\kappa_{n, \lambda}} d t$ of $G_{n}$ on $\sum_{\kappa_{n, \lambda} \in \widehat{K}_{n}^{\dagger}} \int_{-\infty}^{\infty}\left(\mathcal{H}_{n, t, \lambda} \otimes\right.$ $\left.w_{n, t, \lambda} \mathbb{C}\right) d t$.

Proposition 4.21 If $m \geqq n$ and $\kappa_{n, \lambda} \in{\widehat{K_{n}}}^{\dagger}$ then $\kappa_{m, \lambda} \in{\widehat{K_{m}}}^{\dagger}$, and inclusion $\mathbb{C}\left[\mathbb{C}^{n}\right] \hookrightarrow \mathbb{C}\left[\mathbb{C}^{m}\right]$ of polynomials maps the highest weight $\lambda$ space for $\kappa_{n, \lambda}$ onto the the highest weight $\lambda$ space for $\kappa_{m, \lambda}$. 
Proof The group $K_{n}$ acts on $\mathbb{C}^{n}$ by some representation $\gamma_{n}$, so the representation of $K_{n}$ on polynomials of degree $d$ is the symmetric power $S^{d}\left(\gamma_{n}^{*}\right)$. Thus we can compute the set $X_{n, d}$ of highest weights of $K_{n}$ on the space $P_{n, d}$ of polynomials of degree $d$ on $\mathbb{C}^{n}$. Running through the 16 cases of Table 4.15 we see that $X_{n, d} \subset X_{m, d}$. For example (Line 3 of Table 4.15) the representation of $S p(m)$ on polynomials of degree $q$ in $C^{2 m}$ is the irreducible representation with highest weight $q \xi_{m, 1}$, and (Lines 5a and 5b of Table 4.15) the representation of $U(1) \times S O(n)$ on polynomials of degree $q$ in $C^{n}$ is the tensor product of the $-q^{\text {th }}$ power of the usual representation of $U(1)$ by scalars on $\mathbb{C}^{n}$ with the multiplicity-free sum of irreducible representations of $S O(n)$ of highest weights $\left\{\xi_{n, 1}, 3 \xi_{n, 1}, 5 \xi_{n, 1}, \ldots, q \xi_{n, 1}\right\}$ if $q$ is odd, $\left\{0 \xi_{n, 1}, 2 \xi_{n, 1}, 4 \xi_{n, 1}, \ldots, q \xi_{n, 1}\right\}$ if $q$ is even.

Now let $\lambda \in X_{n, d}$. Let $v_{n, \lambda}$ denote a (nonzero) highest weight $\lambda$ vector for $\mathfrak{k}_{n}$ in $P_{n, d}$, and similarly let $v_{m, \lambda}$ denote a (nonzero) highest weight $\lambda$ vector for $\mathfrak{k}_{m}$. Divide up the variables of $\mathbb{C}^{m}$ to $\left\{w_{1}, \ldots, w_{n}\right\}$ for $\mathbb{C}^{n}$ and $\left\{z_{n+1}, \ldots, z_{m}\right\}$ for its complement in $\mathbb{C}^{m}$. Express $v_{m, \lambda}=\sum_{A, B} b_{A, B} w^{A} z^{B}$ where each term has total degree $|A|+|B|=d$. Note that $K_{n}$ treats the $z_{i}$ as constants. Evaluating the $z_{i}$ at arbitrary constant values $C=\left(c_{n+1}, \ldots, c_{m}\right)$ we have a highest weight $\lambda$ vector for $\mathfrak{k}_{n}$. By Carcano's Theorem it is a multiple of $v_{n, \lambda}$. In other words $\left.v_{m, \lambda}\right|_{\{z=C\}}=m_{C} v_{n, \lambda}$. The terms $b_{A, B} \mathbf{w}^{A} \mathbf{z}^{B}$ with $\mathbf{z}$-degree $|B|>0$ yield evaluations of $\mathbf{w}$-degree $|A|<d$, and cannot contribute to any $m_{C} v_{n, \lambda}$. Now $b_{A, B} \mathbf{w}^{A} \mathbf{z}^{B}=0$ whenever $|B|>0$. This shows that $v_{m, \lambda}$ is a homogeneous polynomial of degree $d$ in the $w_{j}$, as is $v_{n, \lambda}$. We conclude that $v_{m, \lambda}$ is a nonzero multiple of $v_{n, \lambda}$.

Corollary 4.22 Condition (2.24) is satisfied for the direct systems $\left\{K_{n}\right\}$ of Table 4.15.

Proof Retain the notation $X_{n, d}$ for those $\lambda$ such that $\kappa_{n, \lambda}$ occurs on the space $P_{n, d}$ of polynomials of degree $d$ on $\mathbb{C}^{n}$. If $\lambda \notin X_{n, d}$ there are no nonzero $K_{n}$-invariant vectors in $\mathcal{H}_{n, t, \lambda}$, so the assertion is vacuous. Now assume $\lambda \in X_{n, d}$ and choose an orthonormal basis $\left\{x_{1}, \ldots, x_{q(n)}\right\}$ of the representation space for $\kappa_{n, \lambda}$ in $P_{n, d}$. According to Proposition 4.21 that representation space is contained in the representation space for $\kappa_{m, \lambda}$ in $P_{m, d}$, so the latter has an orthonormal basis $\left\{x_{1}, \ldots, x_{q(n)}, x_{q(n)+1}, \ldots, x_{q(m)}\right\}$. Let $\left\{x_{1}^{*}, \ldots, x_{q(n)}^{*}, x_{q(n)+1}^{*}, \ldots, x_{q(m)}^{*}\right\}$ and $\left\{x_{1}^{*}, \ldots, x_{q(n)}^{*}\right\}$ be the corresponding dual bases of $\mathcal{F}_{m, \lambda}$ and $\mathcal{F}_{n, \lambda}$. The $K_{n}$-invariant vectors in $\mathcal{H}_{n, t, \lambda}$ are the multiples of $\sum_{1}^{q(n)} x_{i} \otimes x_{i}^{*}$, and the $K_{m}$-invariant vectors in $\mathcal{H}_{m, t, \lambda}$ are the multiples of $\sum_{1}^{q(m)} x_{i} \otimes$ $x_{i}^{*}$. The adjoint of unitary inclusion is orthogonal projection, which sends $\sum_{1}^{q(m)} x_{i} \otimes$ $x_{i}^{*}$ to $\sum_{1}^{q(n)} x_{i} \otimes x_{i}^{*}$.

Let $w_{n, \lambda}$ be a right- $K_{n}$-fixed unit vector in the highest weight $\lambda$ subspace of $L^{2}\left(H_{n} \rtimes\right.$ $K_{n}$ ), for each $\kappa_{n, \lambda} \in{\widehat{K_{n}}}^{\dagger}$. Note that $w_{n, \lambda}$ does not depend on $t$. Proposition 4.21 says that the inclusion $\mathcal{E}_{n, t, \lambda} \hookrightarrow \mathcal{E}_{m, t, \lambda}$ maps $w_{n, \lambda}$ to a nonzero multiple of $w_{m, \lambda}$. Given $\lambda$ we recursively choose the $w_{n, \lambda}$ so that

$$
w_{m, \lambda}=c_{m, n, t, \lambda} w_{n, \lambda}+x \text { with } x \perp \mathcal{E}_{n, t, \lambda} \text { with } 0<c_{n, t, \lambda} \leqq 1 \text {. }
$$


Note $\mathcal{H}_{n, \lambda^{*}}^{K_{n}}=w_{n, \lambda^{*}} \mathbb{C}$ and $\mathcal{H}_{m, \lambda^{*}}^{K_{n}}=w_{m, \lambda^{*}} \mathbb{C}$. Now we have $\left(H_{n} \rtimes K_{n}\right)$-equivariant injections

$$
\alpha_{m, n, t, \lambda^{*}}: \mathcal{H}_{n, t, \lambda^{*}}^{K_{n}} \rightarrow \mathcal{H}_{m, t, \lambda^{*}}^{K_{m}}, \quad \alpha_{m, n, t, \lambda^{*}}\left(w_{n, \lambda^{*}}\right)=c_{m, n, t, \lambda} w_{m, \lambda^{*}},
$$

as in $(2.25)$, and

$$
\begin{gathered}
\widetilde{\zeta}_{m, n, t, \lambda}: \mathcal{H}_{n, t, \lambda} \otimes\left(w_{n, t, \lambda *} \mathbb{C}\right) \rightarrow \mathcal{H}_{m, t, \lambda} \otimes\left(w_{m, t, \lambda} * \mathbb{C}\right) \\
\text { defined by } f \mapsto c_{m, n, t, \lambda} \zeta_{m, n, t, \lambda}(f)
\end{gathered}
$$

as in (2.29) and (2.31). Now as in Section 2.5, $\left\{\mathcal{H}_{n, t, \lambda} \otimes\left(w_{n, \lambda *} \mathbb{C}\right), \widetilde{\zeta}_{m, n, t, \lambda}\right\}$ is isomorphic to a subsystem of the system $\left\{\left(\mathcal{H}_{n, t, \lambda} \widehat{\otimes} \mathcal{H}_{n, t, \lambda}^{*}\right), \zeta_{m, n, t, \lambda}\right\}$ of (3.11). As a result we have

$$
\mathcal{H}_{t, \lambda} \otimes w_{\lambda *} \mathbb{C}:=\lim _{\longrightarrow}\left\{\mathcal{H}_{n, t, \lambda} \otimes w_{n, \lambda *} \mathbb{C}, \widetilde{\zeta}_{m, n, t, \lambda}\right\}
$$

and they fit together under the direct integral (2.23) to give us $L^{2}\left(\left(H_{n} \rtimes K_{n}\right) / K_{n}\right)$ as follows.

$$
L^{2}\left(\left(H_{\infty} \rtimes K\right) / K\right):=L^{2}\left(H_{\infty} \rtimes K\right)^{K}=\lim _{\longrightarrow}\left\{L^{2}\left(\left(H_{n} \rtimes K_{n}\right) / K_{n}\right), \widetilde{\zeta}_{m, n}\right\} .
$$

Combining Theorem 4.17, Lemma 4.20 and Corollary 4.22 we have

Theorem 4.28 Let $\left.\left\{G_{n}, K_{n}\right)\right\}$ be one of the direct systems of Table 4.15. Define $G_{n}=$ $H_{n} \rtimes K_{n}, G=\lim _{\longrightarrow} G_{n}$ and $K=\lim _{\longrightarrow} K_{n}$. Then (4.27) is a unitary direct system whose limit Hilbert space is $G$-isometric to $L^{2}(G)^{K}$, and the natural unitary representation of $G$ on $L^{2}(G / K)=L^{2}(G)^{K}$ is multiplicity free.

Now we turn to regular functions. As in (4.12) we define

$\mathcal{A}\left(H_{n} \rtimes K_{n}\right)$ : finite lin comb in $\ell, \mathbf{m}, i, j, \lambda$ of the $e^{-|t|} p_{\ell, \mathbf{m}, i, j ; n, t, \lambda}(t) f_{\ell, \mathbf{m}, i, j ; n, t, \lambda,}$ $\mathcal{A}\left(\left(H_{n} \rtimes K_{n}\right) / K_{n}\right):=\mathcal{A}\left(H_{n} \rtimes K_{n}\right)^{K_{n}}$,

$v_{m, n, t, \lambda}: \mathcal{A}\left(\pi_{n, t, \lambda}\right)^{K_{n}} \hookrightarrow \mathcal{A}\left(\pi_{m, t, \lambda}\right)^{K_{m}}$ by $f_{u, v_{n}, n} \mapsto f_{u, v_{m}, m}$ where $p_{m, n, t, \lambda}\left(v_{m}\right)=v_{n}$

where the $p_{\ell, \mathbf{m}, i, j ; n, t, \lambda}$ are polynomials. Now we have direct systems and their limits

$$
\begin{aligned}
\mathcal{A}\left(H_{\infty} \rtimes K\right) & =\underset{\longrightarrow}{\lim }\left\{\mathcal{A}\left(H_{n} \rtimes K_{n}\right), \zeta_{m, n}\right\} \text { where } \zeta_{m, n, t, \lambda}: \mathcal{E}_{n, t, \lambda} \hookrightarrow \mathcal{E}_{m, t, \lambda}(4.16), \text { and } \\
\mathcal{A}\left(\left(H_{\infty} \rtimes K\right) / K\right) & =\lim _{\longrightarrow}\left\{\mathcal{A}\left(\left(H_{n} \rtimes K_{n}\right) / K_{n}\right), v_{m, n}\right\} \text { where } v_{m, n, t, \lambda}: \mathcal{E}_{n, t, \lambda}^{K_{n}} \hookrightarrow \mathcal{E}_{m, t, \lambda}^{K_{m}} \text { (4.29) }
\end{aligned}
$$

As before, each $\mathcal{A}\left(\left(H_{n} \rtimes K_{n}\right) / K_{n}\right)$ is a dense subspace of $L^{2}\left(\left(H_{n} \rtimes K_{n}\right) / K_{n}\right)$. In order to pass this comparison to the limit we use the maps

$$
\tilde{\eta}_{n, t, \lambda}: \mathcal{A}\left(\pi_{n, t, \lambda}\right)^{K_{n}} \rightarrow \mathcal{H}_{\pi_{n, t, \lambda}} \otimes\left(w_{n, t, \lambda *} \mathbb{C}\right) \text { by } f \mapsto c_{n, 1, \lambda}|t|^{n / 2} \sqrt{\operatorname{deg} \kappa_{n, \lambda}} f .
$$


Proposition 4.32 The $\widetilde{\eta}_{n, t, \lambda}$ satisfy $\left(\widetilde{\eta}_{m, t, \lambda} \circ v_{m, n, t, \lambda}\right)(f)=\left(\widetilde{\zeta}_{m, n, \lambda} \circ \widetilde{\eta}_{n, t, \lambda}\right)(f)$ for $f \in \mathcal{A}\left(\pi_{n, t, \lambda}\right)^{K_{n}}$ Thus they inject the direct system $\left\{\mathcal{A}\left(\left(H_{n} \rtimes K_{n}\right) / K_{n}\right), v_{m, n}\right\}$ of regular functions into the direct system $\left\{L^{2}\left(\left(H_{n} \rtimes K_{n}\right) / K_{n}\right), \widetilde{\zeta}_{m, n}\right\}$ of square integrable functions. That map of direct systems defines an $\left(H_{\infty} \rtimes K\right)$-equivariant injection

$$
\tilde{\eta}: \mathcal{A}\left(\left(H_{\infty} \rtimes K\right) / K\right) \rightarrow L^{2}\left(\left(H_{\infty} \rtimes K\right) / K\right)
$$

with dense image. In particular $\tilde{\eta}$ defines a pre Hilbert space structure on $\mathcal{A}\left(\left(H_{\infty} \rtimes\right.\right.$ $K) / K)$ with completion isometric to $L^{2}\left(\left(H_{\infty} \rtimes K\right) / K\right)$.

\section{Extension to commutative nilmanifolds}

The results of Sect. 4 depend on four basic facts. First, the $\pi_{n, t}$ are determined by their central character. Second, we have good concrete models $\mathcal{H}_{n, t}$ for the representation spaces, such that $n$ does not appear explicitly in the formulae for the actions of the group elements. Third, the injections $H_{n} \hookrightarrow H_{m}$ restrict to isomorphisms $Z_{n} \cong Z_{m}$ of the centers. And fourth, we have complete information on the Plancherel measure for the $H_{n}$. In this section we consider a somewhat larger class of nilpotent direct systems that satisfy these conditions. In this section we extend our Heisenberg group considerations to nilpotent Lie groups with square integrable representations, following the general lines of [16] and [18].

\subsection{Square integrable nilpotent groups}

Here is a quick summary of harmonic analysis for connected simply connected groups that admit square integrable representations. See [8] for details, [16, Section 14.2] for an exposition. Let $N$ be a connected, simply connected nilpotent Lie group and $\mathfrak{n}$ its Lie algebra. Decompose $\mathfrak{n}=\mathfrak{z}+\mathfrak{v}$ and $N=Z \exp (\mathfrak{v})$ where $\mathfrak{z}$ is the center of $\mathfrak{n}$. Then $Z=\exp (\mathfrak{z})$ is the center of $N$. We say that an irreducible unitary representation $\pi$ of $N$ is square integrable if its coefficient functions $f_{u, v}(g)=\langle u, \pi(g) v\rangle$ satisfy $\left|f_{u, v}\right| \in L^{2}(N / Z)$. In that case $\pi$ is determined by its central character, $\pi=\pi_{t}$ where $t \in \mathfrak{z}^{*}$ and the central character is $\exp (\zeta) \mapsto e^{i t(\zeta)}$. In terms of geometric quantization, $\pi_{t}$ corresponds to the coadjoint orbit in $\mathfrak{n}^{*}$ consisting of all linear functionals on $\mathfrak{n}$ whose restriction to $z$ is $t$. Further, the antisymmetric bilinear form $b_{t}(\xi, \eta)=t([\xi, \eta])$ on $\mathfrak{v}$ is nondegenerate, and (up to a positive constant that depends only on normalizations of Haar measures) the formal degree of $\pi_{t}$ is $\left|\operatorname{Pf}\left(b_{t}\right)\right|$, where $\operatorname{Pf}\left(b_{t}\right)$ is the Pfaffian $^{1}$ of $b_{t}: \mathfrak{v} \times \mathfrak{v} \rightarrow \mathbb{R}$. In fact, if $\pi_{s}$ is the representation of $N$ that corresponds to $\operatorname{Ad}^{*}(N) s \subset \mathfrak{n}^{*}$, then $\pi_{s}$ is square integrable if and only if $\operatorname{Pf}\left(b_{s}\right) \neq 0$. In any case,

$$
\operatorname{Pf}\left(b_{t}\right) \text { is a polynomial function of } t, \operatorname{Pf}\left(b_{t}\right)=P(t) \text {, and } P(0)=0 \text {. }
$$

\footnotetext{
1 Strictly speaking, $\operatorname{Pf}\left(b_{t}\right)$ depends on a choice of basis of $\mathfrak{v}$, for a basis change of determinant $a_{t}$ multiplies $\left.\operatorname{det} b_{t}\right|_{\mathfrak{v} \times \mathfrak{v}}$ by $\operatorname{det} a_{t}^{2}$ and multiplies $\operatorname{Pf}\left(b_{t}\right)$ by $\operatorname{det} a_{t}$.
} 
Again, up to a constant that depends on normalizations, $\left|\operatorname{Pf}\left(b_{t}\right)\right|$ is the Plancherel density. It follows that if one irreducible unitary representation of $N$ is square integrable then Plancherel-almost-all are. In the case of the Heisenberg group $H_{n}$, where we identified $\mathfrak{z}^{*}$ with $\mathbb{R}$, the Pfaffian corresponding to $\pi_{n, t}$ is $t^{n}$.

When we are dealing with a sequence $\left\{N_{n}\right\}$ if square integrable nilpotent groups, we have to keep track of the polynomials (5.1), so we will write

$$
P(n, t)=\operatorname{Pf}\left(b_{n, t}\right): \text { corresponding to the group } N_{n} .
$$

The point of this, from the viewpoint of commutative spaces, is that many Gelfand pairs are of the form $(N \rtimes K, K)$ where $N$ is a connected simply connected Lie group, $K$ is a compact subgroup of $\operatorname{Aut}(N)$, and $N$ has square integrable representations. See [16, Theorem 14.4.3]. This is simplified by the 2-step Nilpotent Theorem [16, Theorem 13.1.1] of Benson-Jenkins-Ratcliff and Vinberg, which says that $N$ must be abelian or 2-step nilpotent. In a certain sense representations treat those groups as Heisenberg groups:

Lemma 5.3 [16, Lemma 14.4.1] Let $N$ be a connected simply connected 2-step nilpotent Lie group with 1-dimensional center. Then $N$ is isomorphic to the Heisenberg group $H_{n}$ where $n=\frac{1}{2}\left(\operatorname{dim}_{\mathbb{R}} \mathfrak{n}-1\right)$, and in particular $N$ has square integrable representations.

Proposition 5.4 [16, Proposition 14.4.2] Let $N$ be a connected simply connected 2-step nilpotent Lie group. Let $f \in \mathfrak{n}^{*}$ such that $\left.f\right|_{\mathfrak{z}} \neq 0$. Denote $\mathfrak{w}_{f}=\{z \in \mathfrak{z} \mid$ $f(z)=0\}$ and $W_{f}:=\exp \left(\mathfrak{w}_{f}\right)$. Then

1. $W_{f}$ is a closed subgroup of $Z$, hence a closed normal subgroup of $N$.

2. The functional $f$ is the pullback of a linear functional $f^{\prime} \in\left(\mathfrak{n} / \mathfrak{w}_{f}\right)^{*}$ and is nonzero on the central subalgebra $\mathfrak{z} / \mathfrak{w}$ of $\mathfrak{n} / \mathfrak{w}{ }_{f}$.

3. The representation $\left[\pi_{f}\right]$ is the pullback to $N$ of the class $\left[\pi_{f^{\prime}}\right] \in \widehat{N / W_{f}}$.

4. If the representation $\left[\pi_{f}\right]$ is square integrable then $\left[\pi_{f^{\prime}}\right]$ is square integrable, and in that case $N / W_{f}$ has center $Z / W_{f}$ and is isomorphic to a Heisenberg group $H_{n}$ where $n=\frac{1}{2} \operatorname{dim}(\mathfrak{n} / \mathfrak{z})$.

We now consider a strict direct system $\left\{N_{n}\right\}$ of 2-step nilpotent connected, simply connected Lie groups that have square integrable representations, where the inclusions $\mathfrak{n}_{n} \rightarrow \mathfrak{n}_{m}$ map the center $\mathfrak{z}_{n} \hookrightarrow \mathfrak{z}_{m}$ and the complement $\mathfrak{v}_{n} \hookrightarrow \mathfrak{v}_{m}$ in decompositions $\mathfrak{n}_{n}=\mathfrak{z}_{n}+\mathfrak{v}_{n}$. Then the direct limit algebra $\mathfrak{n}:=\underline{\lim } \mathfrak{n}_{n}$ has center $\mathfrak{z}:=\lim _{\longrightarrow} \mathfrak{z}_{n}$ and $\mathfrak{n}=\mathfrak{z}+\mathfrak{v}$ where $\mathfrak{v}=\lim _{\longrightarrow} \mathfrak{v}_{n}$. On the group level, $\overrightarrow{Z \longrightarrow} \lim _{n} Z_{n}=\exp (\mathfrak{z})$ is $\overrightarrow{\text { the center }}$ of $N:=\lim N_{n}$ and we have $N=Z \exp (\mathfrak{v})$.

We further assume that the dimensions $\operatorname{dim} Z_{n}$ of the centers are bounded. Since they are non-decreasing we may assume that they are eventually constant. Passing to a cofinal sequence,

$$
\mathfrak{n}_{n} \hookrightarrow \mathfrak{n}_{m} \text { maps } \mathfrak{z}_{n} \cong \mathfrak{z}_{m}
$$

Under that identification we write $\mathfrak{z}$ for all the $\mathfrak{z}_{n}, \mathfrak{z}^{*}$ for all the $\mathfrak{z}_{n}^{*}$, and $Z$ for all the $Z_{n}$. 
Let $t \in \mathfrak{z}^{*}$. Write $b_{n, t}$ for the bilinear form $(\xi, \eta) \mapsto t([\xi, \eta])$ on $\mathfrak{v}_{n}$. Then $t$ corresponds to a square integrable representation $\pi_{n, t}$ of $N_{n}$ just when the Pfaffian $\operatorname{Pf}\left(b_{n, t}\right) \neq 0$. For purposes of comparing the Pfaffians as $n$ varies, we note that $\operatorname{Pf}\left(b_{n, t}\right)$ is specified by $t$ and a basis of $\mathfrak{v}_{n}$, so we simply assume that these bases are nested in the sense that the basis of $\mathfrak{v}_{n+1}$ consists of the basis of $\mathfrak{v}_{n}$ together with some elements that are $b_{n+1, t}$-orthogonal to $\mathfrak{v}_{n}$. Thus, if $\operatorname{Pf}\left(b_{n+1, t}\right) \neq 0$ then $\operatorname{Pf}\left(b_{n, t}\right) \neq 0$. The converse fails in general, but the following lemma deals with the possibility that $\operatorname{Pf}\left(b_{n, t}\right) \neq 0=\operatorname{Pf}\left(b_{m, t}\right)$. It depends on the fact [8] that each $\operatorname{Pf}\left(b_{n, t}\right)$ is a polynomial function on $\mathfrak{z}^{*}$.

Lemma 5.6 Let $\mathfrak{a}_{n} \in \mathfrak{z}^{*}$ denote the zero set of $\operatorname{Pf}\left(b_{n, t}\right)$ and set $\mathfrak{a}=\bigcup \mathfrak{a}_{n}$. Then $\mathfrak{a}$ is a set of Lebesgue measure zero in $\mathfrak{z}^{*}$.

Proof Since $N_{n}$ has square integrable representations, the $\operatorname{Pfaffian} \operatorname{Pf}\left(b_{n, t}\right)$ is a nontrivial polynomial function of $t \in \mathfrak{z}^{*}$, so $\mathfrak{a}_{n}$ is a finite union of lower-dimensional subvarieties of $\mathfrak{z}_{n}^{*}$. Now the set $\mathfrak{a}$ is a countable union of sets $\mathfrak{a}_{n}$ of Lebesgue measure zero.

Define $T=\left\{t \in \mathfrak{z}^{*} \mid\right.$ each $\left.\operatorname{Pf}\left(b_{n, t}\right) \neq 0\right\}$. So $\mathfrak{z}^{*} \backslash \mathfrak{a}$. For every $t \in T$ and every index $n$ we have a square integrable representation $\pi_{n, t} \in \widehat{N}_{n}$. Let $t \in T, \mathfrak{w}_{t}=\{z \in$ $\mathfrak{z} \mid t(z)=0\}$ and $W_{t}=\exp \left(\mathfrak{w}_{t}\right)$. Then $W_{t}$ is closed in $Z, N_{n} / W_{t}$ is isomorphic to a Heisenberg group $H_{d(n)}$, and $\pi_{n, t}$ factors through to the square integrable representation of $N_{n} / W_{t}$ with central character $e^{i t}$. As $t$ varies in $T$ the $\pi_{n, t}$ act on the same Fock space $\mathcal{H}_{d(n), t}, d(n)=\frac{1}{2} \operatorname{dim} \mathfrak{v}_{n}$, by formulae independent of $d(n)$.

We normalize the inner products on the $\mathcal{H}_{d(n), t}$ as before, so the $\mu_{\mathbf{m}}$ form a complete orthonormal set, and realize the space $\mathcal{E}_{n, t}=\mathcal{H}_{d(n), t} \widehat{\otimes} \mathcal{H}_{d(n), t}^{*}$ of matrix coefficients as the closed span of the functions $f_{\ell, \mathbf{m} ; n, t}: g \mapsto\left\langle\mu_{\ell}, \pi_{n, t}(g) \mu_{\mathbf{m}}\right\rangle$, as in Sect. 4. The orthogonality relations say that the inner product on $\mathcal{E}_{n, t}$ is given by $\left\langle f_{\ell, \mathbf{m} ; n, t}, f_{\ell^{\prime}, \mathbf{m}^{\prime} ; n, t}\right\rangle=\left|\operatorname{Pf}\left(b_{n, t}\right)\right|^{-1}$ if $\ell=\ell^{\prime}$ and $\mathbf{m}=\mathbf{m}^{\prime}$, and is 0 otherwise. Now the $\left|\operatorname{Pf}\left(b_{n, t}\right)\right|^{1 / 2} f_{\ell, \mathbf{m} ; n, t}$ form a complete orthonormal set in $\mathcal{E}_{n, t}$, and as before $\mathcal{E}_{n, t}$ consists of the functions $\Phi_{n, t, \varphi}$ on $H_{d(n)}$ given by

$$
\Phi_{n, t, \varphi}(h)=\sum_{\ell, \mathbf{m}} \varphi_{\ell, \mathbf{m}}(t)\left|\operatorname{Pf}\left(b_{n, t}\right)\right|^{1 / 2} f_{\ell, \mathbf{m} ; n, t}(h) \text { with } \sum_{\ell, \mathbf{m}}\left|\varphi_{\ell, \mathbf{m}}(t)\right|^{2}<\infty .
$$

Now $L^{2}\left(N_{n}\right)=\int_{\mathfrak{z}_{n}^{*}} \mathcal{E}_{n, t}\left|\operatorname{Pf}\left(b_{n, t}\right)\right| d t=\int_{T} \mathcal{E}_{n, t}\left|\operatorname{Pf}\left(b_{n, t}\right)\right| d t$. It consists of all functions $\Psi_{n, \varphi}$ defined by

$$
\begin{aligned}
\Psi_{n, \varphi}(h) & =\int_{\mathfrak{z}_{n}^{*}} \Phi_{n, t, \varphi}(h)\left|\operatorname{Pf}\left(b_{n, t}\right)\right| d t \\
& =\int_{T}\left(\sum_{\ell, \mathbf{m}} \varphi_{\ell, \mathbf{m}}(t)\left|\operatorname{Pf}\left(b_{n, t}\right)\right|^{1 / 2} f_{\ell, \mathbf{m} ; n, t}\right)\left|\operatorname{Pf}\left(b_{n, t}\right)\right| d t
\end{aligned}
$$

such that the functions $\varphi_{\ell, \mathbf{m}}: \mathfrak{z}_{n}^{*} \rightarrow \mathbb{C}$ are measurable with $\sum_{\ell, \mathbf{m}}\left|\varphi_{\ell, \mathbf{m}}(t)\right|^{2}<\infty$ for almost all $t \in T$ and $\sum_{\ell, \mathbf{m}}\left|\varphi_{\ell, \mathbf{m}}(t)\right|^{2} \in L^{1}\left(\mathfrak{z}_{n}^{*},\left|\operatorname{Pf}\left(b_{n, t}\right)\right| d t\right)$. The norms are 


$$
\begin{aligned}
\left\|\Psi_{n, \varphi}\right\|_{L^{2}\left(N_{n}\right)}^{2} & =\int_{T}\left\|\Phi_{n, t, \varphi}\right\|_{\mathcal{E}_{n, t}}^{2}\left|\operatorname{Pf}\left(b_{n, t}\right)\right| d t=\int_{T}\left(\sum_{\ell, \mathbf{m}}\left|\varphi_{\ell, \mathbf{m}}(t)\right|^{2}\right)\left|\operatorname{Pf}\left(b_{n, t}\right)\right| d t \\
& =\sum_{\ell, \mathbf{m}}\left\|\varphi_{\ell, \mathbf{m}}\right\|_{L^{2}\left(\mathfrak{z}^{*},\left|\operatorname{Pf}\left(b_{n, t}\right)\right| d t\right)}^{2}
\end{aligned}
$$

The left/right representation of $N_{n} \times N_{n}$ on $\mathcal{E}_{n, t}$ is the exterior tensor product $\pi_{n, t} \otimes \pi_{n, t}^{*}$; it is irreducible and the left/right regular representation of $N_{n} \times N_{n}$ on $L^{2}\left(N_{n}\right)$ is the multiplicity free unitary representation $\int_{\mathfrak{z}^{*}}\left(\pi_{n, t} \otimes \pi_{n, t}^{*}\right)\left|\operatorname{Pf}\left(b_{n, t}\right)\right| d t$.

Let $m \geqq n$. Then $\left|\operatorname{Pf}\left(b_{n, t}\right)\right|^{1 / 2} f_{\ell, \mathbf{m} ; n, t} \mapsto\left|\operatorname{Pf}\left(b_{m, t}\right)\right|^{1 / 2} f_{\ell, \mathbf{m} ; m, t}$ defines an equivariant isometric injection $\Phi_{n, \varphi}(t) \mapsto \Phi_{m, \varphi}(t)$ of $\mathcal{E}_{n, t}$ into $\mathcal{E}_{m, t}$. The norm computation just above gives

$$
\left\|\Psi_{m,\left|\operatorname{Pf}\left(b_{n, t}\right) / \operatorname{Pf}\left(b_{m, t}\right)\right|^{1 / 2} \varphi}\right\|_{L^{2}\left(N_{m}\right)}^{2}=\left\|\Psi_{n, \varphi}\right\|_{L^{2}\left(N_{n}\right)}^{2} .
$$

Thus we have an $\left(N_{n} \times N_{n}\right)$-equivariant isometric injection

$$
\zeta_{m, n}: L^{2}\left(N_{n}\right) \rightarrow L^{2}\left(N_{m}\right) \text { by } \zeta_{m, n}\left(\Psi_{n, \varphi}\right)=\Psi_{m,\left|\operatorname{Pf}\left(b_{n, t}\right) / \operatorname{Pf}\left(b_{m, t}\right)\right|^{1 / 2} \varphi}
$$

On the level of coefficients it is given by $\zeta_{m, n}\left(\Phi_{n, t, \varphi}\right)=\Phi_{m, t,\left|\operatorname{Pf}\left(b_{n, t}\right) / \operatorname{Pf}\left(b_{m, t}\right)\right|^{1 / 2} \varphi}$. In other words $\zeta_{m, n}$ sends the function $\sum_{\ell, \mathbf{m}} \varphi_{\ell, \mathbf{m}}(t)\left|\operatorname{Pf}\left(b_{n, t}\right)\right|^{1 / 2} f_{\ell, \mathbf{m} ; n, t}$ on $N_{n}$ to the function on $N_{m}$ given by

$$
\begin{aligned}
& \sum_{\ell, \mathbf{m}}\left(\left|\operatorname{Pf}\left(b_{n, t}\right) / \operatorname{Pf}\left(b_{m, t}\right)\right|^{1 / 2} \varphi_{\ell, \mathbf{m}}(t)\right)\left(\left|\operatorname{Pf}\left(b_{m, t}\right)\right|^{1 / 2} f_{\ell, \mathbf{m} ; m, t}\right) \\
& \quad=\sum_{\mathbf{l}, \mathbf{m}} \varphi_{\ell, \mathbf{m}}(t)\left|\operatorname{Pf}\left(b_{n, t}\right)\right|^{1 / 2} f_{\ell, \mathbf{m} ; t} .
\end{aligned}
$$

The result is

Theorem 5.12 There is a strict direct system $\left\{L^{2}\left(N_{n}\right), \zeta_{m, n}\right\}$ of $L^{2}$ spaces. The direct system maps $\zeta_{m, n}: L^{2}\left(N_{n}\right) \rightarrow L^{2}\left(N_{m}\right)$ are $\left(N_{n} \times N_{n}\right)$-equivariant unitary injections. Let $\Pi_{n}$ denote the left/right regular representation of $N_{n} \times N_{n}$ on $L^{2}\left(N_{n}\right)$ and let $N=\underline{\lim } N_{n}$. Then we have a well defined Hilbert space $L^{2}(N):=\lim _{\longrightarrow}\left\{L^{2}\left(N_{n}\right), \zeta_{m, n}\right\}$ and a multiplicity-free unitary representation $\Pi=\lim _{\longrightarrow} \Pi_{n}$ of $N \times \vec{N}$ on $L^{2}(N)$.

\subsection{Semidirect product groups}

We need some structural results from [18, Section 7] for a strict direct system $\left\{K_{n}, \varphi_{m, n}\right\}$ of compact connected Lie groups and a consistent family $\left\{\gamma_{n}\right\}$ of representations of the $K_{n}$ on a fixed finite dimensional vector space $\mathfrak{z}$. We'll use that information to extend Theorems 4.17 and 4.28 to a larger family of strict direct systems of nilmanifold Gelfand pairs.

As just indicated, $\left\{K_{n}, \varphi_{m, n}\right\}$ is a strict direct system of compact connected Lie groups. Denote $K=\lim _{\longrightarrow}\left\{K_{n}, \varphi_{m, n}\right\}$ and let $\left\{\gamma_{n}\right\}$ be a consistent family of unitary representations of the $K_{n}$ on a fixed finite dimensional real vector space $\mathfrak{z}$. The $U_{n}=\gamma_{n}\left(K_{n}\right)$ 
form an increasing sequence of compact connected subgroups of dimension $\leqq(\operatorname{dim} \mathfrak{z})^{2}$ in the orthogonal group $O(\mathfrak{z})$, so from some index on they are all the same compact connected subgroup $U$ of $O(\mathfrak{z})$. Now we truncate the index set and assume that the $\gamma_{n}$ all have the same image $U$ in the orthogonal group of $\mathfrak{z}$.

Let $K_{n}^{\dagger}$ denote the identity component of the kernel of $\gamma_{n}$. Then $\varphi_{m, n}\left(K_{n}^{\dagger}\right) \subset K_{m}^{\dagger}$, so we have $K^{\dagger}=\underline{\lim _{\longrightarrow}}\left\{K_{n}^{\dagger},\left.\varphi_{m, n}\right|_{K_{n}^{\dagger}}\right\}$, and $K^{\dagger}$ is the identity component of the kernel of $\gamma$. Since $K_{n}$ is compact and connected, and $K_{n}^{\dagger}$ is a closed connected normal subgroup, $K_{n}$ has another closed connected normal subgroup $L_{n}$ such that $K_{n}$ is locally isomorphic to the direct product $K_{n}^{\dagger} \times L_{n}$.

Proposition 5.13 One can choose the groups $L_{n}$ so that $\varphi_{m, n}\left(L_{n}\right)=L_{m}$ for $m \geqq$ $n \gg 0$.

Let $L=\lim _{\longrightarrow}\left\{L_{n},\left.\varphi_{m, n}\right|_{L_{n}}\right\}$. We further truncate the index set so that $L_{n}=\varphi_{n}^{-1}(L)$ for all $n$.

Corollary 5.14 Let $t \in \mathfrak{z}$, and let $K_{n, t}$ be its stabilizer in $K_{n}$. If one of the direct systems $\left\{K_{n}, \varphi_{m, n}\right\},\left\{K_{n, t},\left.\varphi_{m, n}\right|_{K_{n, t}}\right\}$, or $\left\{K_{n}^{\dagger},\left.\varphi_{m, n}\right|_{K_{n}^{\dagger}}\right\}$ is parabolic, then the other two also are parabolic.

Corollary 5.15 Let $t \in \mathfrak{z}$, and let $K_{n, t}$ be its stabilizer in $K_{n}$. Suppose that the direct system $\left\{K_{n}, \varphi_{m, n}\right\}$ is parabolic. Then there are natural isometric injections $\mathcal{F}_{n, t, \lambda} \hookrightarrow$ $\mathcal{F}_{m, t, \lambda}$ for $m \geqq n$, from the highest weight $\lambda$ representation space of $K_{n, t}$ to that of $K_{m, t}$, and corresponding $L^{2}$-isometric injections $f \mapsto\left(\left(\operatorname{deg} \kappa_{m, t, \lambda}\right) /\left(\operatorname{deg} \kappa_{n, t, \lambda}\right)\right)^{1 / 2} f$ on spaces of coefficient functions.

Corollary 5.16 Let $t \in \mathfrak{z}$, and let $K_{n, t}$ be its stabilizer in $K_{n}$. Then $L:=\lim _{\longrightarrow} L_{n}$ is compact, $K=K^{\dagger} L$ where $K^{\dagger}:=\lim _{\rightarrow} K_{n}^{\dagger}$, and $K$ is locally isomorphic to $K^{\dagger} \times L$. In particular $K$ acts on $\mathfrak{z}$ as a compact linear group and $\mathfrak{z}$ has a $\gamma(K)$-invariant positive definite inner product.

Now we can proceed along the lines of [18, Section 8]. Fix a strict direct system $\left\{\left(G_{n}, K_{n}\right)\right\}$ of Gelfand pairs that satisfies

(i) $G_{n}=N_{n} \rtimes K_{n}$, semidirect product, where $N_{n}$ is a connected, simply connected, nilpotent Lie group with square integrable representations and $K_{n}$ is connected,

(ii) the $K_{n}$ form a parabolic strict direct system,

(iii) the inclusions $\mathfrak{n}_{n} \hookrightarrow \mathfrak{n}_{n+1}$ map centers $\mathfrak{z}_{n} \cong \mathfrak{z}_{n+1}$ and map complements

$$
\mathfrak{v}_{n} \hookrightarrow \mathfrak{v}_{n+1},
$$

(iv) for each $n$ the complement $\mathfrak{v}_{n}$ is $\operatorname{Ad}\left(K_{n}\right)$-invariant.

Using (5.17(iii)) we identify each $\mathfrak{z}_{n}$ with $\mathfrak{z}:=\lim _{\mathfrak{z}} \mathfrak{z}_{n}$. Write $K_{n}^{\dagger}$ for the identity component of the kernel of the action of $K_{n}$ on $\mathfrak{z}$. Since $\left.\vec{A} d\left(K_{n}\right)\right|_{\mathfrak{z}}$ is a compact connected connected group of linear transformations of $\mathfrak{z}$, its dimension is bounded, so the $\left.\operatorname{Ad}\left(K_{n}\right)\right|_{\mathfrak{z}}$ stabilize and we may assume that each $\left.\operatorname{Ad}\left(K_{n}\right)\right|_{\mathfrak{z}}=U$ for some compact connected 
group $U$ of linear transformations of $\mathfrak{z}$. Proposition 5.13 gives us complementary closed connected normal subgroups $L_{n} \subset K_{n}$ that inject isomorphically under $K_{n} \hookrightarrow K_{n+1}$, so each $L_{n}$ is equal to $L:=\lim _{\longrightarrow} L_{n}$. Thus we have decompositions $K_{n}=K_{n}^{\dagger} \cdot L$ and $K=K^{\dagger} \cdot L$ where $K_{n}^{\dagger}$ is the kernel of the adjoint action of $K_{n}$ on $\mathfrak{z}, K=\lim _{\longrightarrow} K_{n}$, and $K^{\dagger}=\lim _{\longrightarrow} K_{n}^{\dagger}$. For each $n, \operatorname{Ad}_{K_{n}}$ maps $L=L_{n}$ onto $U$ with finite kernel.

If $t \vec{\in}^{*}$ denote $\mathcal{O}_{t}=\operatorname{Ad}^{*}(L)(t)$, and let $G_{n, t}, K_{n, t}$ and $L_{n, t}$ denote the respective stabilizers of $t$ in $G_{n}, K_{n}$ and $L_{n}$. Since $\operatorname{Ad}^{*}\left(G_{n}\right)$ acts on $\mathcal{O}_{t}$ as the compact group $L$ there is an invariant measure $v_{t}$ derived from euclidean measure on $\mathfrak{z}^{*}$. Given $t \in \mathfrak{z}^{*}$ its stabilizers $G_{t}=\left\{g \in G \mid \operatorname{Ad}^{*}(g) t=t\right\}, K_{t}=K \cap G_{t}$ and $L_{t}=L \cap G_{t}$. Their pullbacks in $G_{n}$ are $G_{n, t}, K_{n, t}$ and $L_{n, t}$. Note that $K_{t}=K^{\dagger} \cdot L_{t}$ and $K_{n, t}=K_{n}^{\dagger} \cdot L_{n, t}$.

Recall $T=\left\{t \in \mathfrak{z}^{*} \mid\right.$ each $\left.\operatorname{Pf}\left(b_{n, t}\right) \neq 0\right\}$ and fix $t \in T$. Essentially as in the Heisenberg group case, the square integrable representation $\pi_{n, t}$ extends to a unitary representation $\widetilde{\pi_{n, t}}$ of $G_{n, t}:=N_{n} \rtimes K_{n, t}$ on the same representation space $\mathcal{H}_{n, t}$. If $\kappa_{n, t, \lambda} \in \widehat{K_{n, t}}$ has representation space $\mathcal{F}_{n, t, \lambda}$ we write $\widetilde{\kappa_{n, t, \lambda}}$ for its extension to a representation of $G_{n, t}$ on $\mathcal{F}_{n, t, \lambda}$ that annihilates $N_{n}$. Then we have the irreducible unitary representations$$
\pi_{n, t, \lambda}^{\diamond}:=\widetilde{\pi_{n, t}} \otimes \widetilde{\kappa_{n, t, \lambda}} \in \widehat{G_{n, t}} \text { with representation space } \mathcal{H}_{n, t, \lambda}^{\diamond}:=\mathcal{H}_{n, t} \otimes \mathcal{F}_{n, t, \lambda}
$$

That gives us the unitarily induced representations

$$
\begin{aligned}
& \pi_{n, t, \lambda}=\operatorname{Ind}_{G_{n, t}}^{G_{n}}\left(\pi_{n, t, \lambda}^{\diamond}\right) \in \widehat{G_{n}} \\
& \quad \text { with representation space } \mathcal{H}_{n, t, \lambda}:=\int_{\mathcal{O}_{t}}\left(\mathcal{H}_{n, \operatorname{Ad}^{*}(k) t} \otimes \mathcal{F}_{n, \operatorname{Ad}^{*}(k) t, \operatorname{Ad}^{*}(k) \lambda}\right) d v_{t}(k(t)) .
\end{aligned}
$$

According to the Mackey little group theory, (i) $\pi_{n, t, \lambda}$ is irreducible, (ii) $\pi_{n, t, \lambda}$ is equivalent to $\pi_{n, t^{\prime}, \lambda^{\prime}}$ if and only if $t^{\prime} \in \mathcal{O}_{t}$, say $t^{\prime}=\operatorname{Ad}^{*}(\ell) t$ where $\ell \in L$, and $\operatorname{Ad}^{*}(\ell)$ carries $\lambda$ to $\lambda^{\prime}$, and (iii) Plancherel-almost-all irreducible unitary representations of $G_{n}$ are of the form $\pi_{n, t, \lambda}$ where $t \in T$ and $\kappa_{n, t, \lambda} \in \widehat{K_{n, t}}$. So the left/right regular representation of $G_{n}$

$$
\begin{aligned}
& \Pi_{n}=\sum_{\lambda} \int_{\mathrm{Ad}^{*}\left(K_{n}\right) \backslash \mathfrak{z}^{*}}\left(\pi_{n, t, \lambda} \otimes \pi_{n, t, \lambda}^{*}\right) d\left(\operatorname{Ad}^{*}\left(K_{n}\right)(t)\right) \\
& \quad \text { with representation space } L^{2}\left(G_{n}\right)=\sum_{\lambda} \int_{\operatorname{Ad}^{*}\left(K_{n}\right) \backslash \mathfrak{z}^{*}}\left(\mathcal{H}_{n, t, \lambda} \widehat{\otimes} \mathcal{H}_{n, t, \lambda}^{*}\right) d\left(\operatorname{Ad}^{*}\left(K_{n}\right)(t)\right) .
\end{aligned}
$$

Since $\pi_{n, t, \lambda}^{\diamond}$ is square integrable and $\pi_{n, t, \lambda}$ is irreducible, one knows [18, Theorem A.1] that $\pi_{n, t, \lambda}$ has a well defined formal degree. Making use of Corollaries 5.14, 5.15 and 5.16, we have $G_{n}$-equivariant isometric injections 
$\zeta_{m, n}: L^{2}\left(G_{n}\right) \rightarrow L^{2}\left(G_{m}\right)$ based on the $\zeta_{m, n, t, \lambda}: \mathcal{H}_{n, t, \lambda} \widehat{\otimes} \mathcal{H}_{n, t, \lambda}^{*} \rightarrow \mathcal{H}_{m, t, \lambda} \widehat{\otimes} \mathcal{H}_{m, t, \lambda}^{*}$ where $\zeta_{m, n, t, \lambda}\left(\left(\operatorname{deg} \pi_{n, t, \lambda}\right)^{1 / 2} f\right)=\left(\operatorname{deg} \pi_{m, t, \lambda}\right)^{1 / 2} f$.

With $G:=\lim _{\longrightarrow} G_{n}$ we now have the

$$
\begin{aligned}
\text { left/right regular representation } \Pi: & :=\lim _{\longrightarrow} \Pi_{n} \text { of } G \times G \text { on } L^{2}(G) \\
& :=\underline{\longrightarrow}\left\{L^{2}\left(G_{n}\right), \zeta_{m, n}\right\}
\end{aligned}
$$

Now we turn to regular functions. As one might expect, $\mathcal{E}_{n, t, \lambda}^{\diamond}$ means $\mathcal{H}_{n, t, \lambda}^{\diamond} \widehat{\otimes}$ $\left(\mathcal{H}_{n, t, \lambda}^{\diamond}\right)^{*}$ viewed as matrix coefficients of $\pi_{n, t, \lambda}^{\diamond}$. As in the (4.18) we define

$\mathcal{A}\left(G_{n, t}\right):=\left\{\right.$ finite linear combinations of the $e^{-\|t\|} p_{\ell, \mathbf{m}, i, j ; n, t, \lambda} f_{\ell, \mathbf{m}, i, j ; n, t, \lambda}$ in $\left.\mathcal{E}_{n, t, \lambda}^{\diamond}\right\}$, $\mathcal{A}\left(G_{n, t}\right) \rightarrow \mathcal{A}\left(G_{m, t}\right)$ by $e^{-|| t||} f_{\ell, \mathbf{m}, i, j ; n, t, \lambda} \mapsto e^{-\| t||} f_{\ell, \mathbf{m}, i, j ; m, t, \lambda}$, and $\mathcal{A}\left(G_{t}\right)$

$$
=\lim _{\longrightarrow} \mathcal{A}\left(G_{n, t}\right)
$$

where the $p_{\ell, \mathbf{m}, i, j ; n, t, \lambda}(t)$ are polynomials. The norm $\|t\|$ on $\mathfrak{z}^{*}$ is from a $U$-invariant inner product.

The representation space $\mathcal{H}_{n, t, \lambda}$ of $\pi_{n, t, \lambda}=$ Ind ${ }_{G_{n, t}}^{G_{n}}\left(\pi_{n, t, \lambda}^{\diamond}\right)$ is the space of $L^{2}$ sections of the Hilbert space bundle $\mathbb{H}_{n, t, \lambda}^{\diamond} \rightarrow \mathcal{O}_{t}=K_{n} / K_{n, t}$. We express it as the space $\left(L^{2}\left(K_{n}\right) \otimes \mathcal{F}_{n, t, \lambda} \otimes \mathcal{H}_{n, t}\right)^{K_{n, t}}$ of $K_{n, t}$-invariants in $L^{2}\left(K_{n}\right) \otimes \mathcal{F}_{n, t, \lambda} \otimes \mathcal{H}_{n, t}$. Let $\mathcal{H}_{n, t}^{\text {poly }}$ denote the space of finite linear combinations of the $\mu_{\mathbf{m}}$ in the space $\mathcal{H}_{n, t}$. Then the underlying algebraic space is the space $\mathcal{A}\left(\pi_{n, t, \lambda}\right)^{K_{n, t}}:=\left(\mathcal{A}\left(K_{n}\right) \otimes \mathcal{F}_{n, t, \lambda} \otimes \mathcal{H}_{n, t}^{\text {poly }}\right)^{K_{n, t}}$ of $K_{n, t}$-invariants in $\mathcal{A}\left(K_{n}\right) \otimes \mathcal{F}_{n, t, \lambda} \otimes \mathcal{H}_{n, t}^{\text {poly }}$. With that and (5.23) in mind we define

$$
\begin{aligned}
\mathcal{A}\left(G_{n}\right):= & \left\{\text { finite linear comb of the } e ^ { - \| t \| } p ( t ) f _ { n , t , \lambda } \text { where } f _ { n , t , \lambda } \in \left(\mathcal{A}\left(K_{n}\right)\right.\right. \\
& \left.\left.\otimes \mathcal{F}_{n, t, \lambda} \otimes \mathcal{H}_{n, t}^{\mathrm{poly}}\right)^{K_{n, t}}\right\} \\
\mathcal{A}\left(G_{n}\right) \rightarrow & \mathcal{A}\left(G_{m}\right) \text { by } e^{-\|t\|} p(t) f_{\ell, \mathbf{m}, i, j ; n, t, \lambda} \mapsto e^{-\|t\|} p(t) f_{\ell, \mathbf{m}, i, j ; m, t, \lambda} \\
& \text { and } \mathcal{A}(G)=\underline{\lim }_{\longrightarrow} \mathcal{A}\left(G_{n}\right)
\end{aligned}
$$

where the $p(t)$ are real polynomials on $\mathfrak{z}^{*}$. The maps corresponding to those of (2.21) are the

$$
\begin{aligned}
\eta_{n, t, \lambda}: \mathcal{A}\left(\pi_{n, t, \lambda}\right) & \rightarrow \mathcal{E}_{n, t, \lambda} \text { by } \eta_{n, t, \lambda}\left(e^{-\|t\|} p(t) f_{\ell, \mathbf{m}, i, j ; t, \lambda}\right) \\
& =e^{-\|t\|} \sqrt{\operatorname{deg} \pi_{n, t, \lambda}} p(t) f_{\ell, \mathbf{m}, i, j ; t, \lambda}
\end{aligned}
$$

Proposition 2.22 now tells us that

Proposition 5.25 The maps $\eta_{m, t, \lambda}$ satisfy $\eta_{m, t, \lambda} \circ \zeta_{m, n, t, \lambda}=\zeta_{m, n, t, \lambda} \circ \eta_{n, t, \lambda}$ on $\mathcal{A}\left(\pi_{n, t, \lambda}\right)$ and send the direct system $\left\{\mathcal{A}\left(G_{n}\right)\right\}$ into the direct system $\left\{L^{2}\left(G_{n}\right), \zeta_{m . n}\right\}$. That system map defines an $(G \times G)$-equivariant injection $\eta: \mathcal{A}(G) \rightarrow L^{2}(G)$ 
with dense image. In particular $\eta$ defines a pre Hilbert space structure on $\mathcal{A}(G)$ with completion isometric to $L^{2}(G)$.

\subsection{Commutative quotients}

We modify the results of Sect. 5.2 to strict direct systems of commutative spaces that satisfy (5.17). Here the $L^{2}$ results are recalled from [18, Sect. 9].

Theorem 5.26 Let $t \in T$. Then $\left(G_{n, t}, K_{n, t}\right)$ is a Gelfand pair. In particular $K_{n, t}$ is multiplicity free on $\mathbb{C}\left[\mathfrak{v}_{n}\right]$.

We have already used Hilbert bundle model for the induced representation $\pi_{n, t, \lambda} \in$ $\widehat{G_{n, t}}$, given by $\pi_{n, t, \lambda}=$ Ind $G_{n, t}^{G_{n}}\left(\pi_{n, t, \lambda}^{\diamond}\right)$. The representation space $\mathcal{H}_{n, t, \lambda}$ of $\pi_{n, t, \lambda}$ consists of all $L^{2}\left(K_{n} / K_{n, t}\right)$ sections of the homogeneous bundle $p: \mathbb{H}_{n, t, \lambda}^{\diamond} \rightarrow$ $G_{n} / G_{n, t}=K_{n} / K_{n, t}$ whose typical fiber is the representation space $\mathcal{H}_{n, t, \lambda}^{\diamond}$ of $\pi_{n, t, \lambda}^{\diamond}$. Given $k \in K_{n}$ we write $k \cdot \mathcal{H}_{n, t, \lambda}^{\diamond}$ for the fiber $p^{-1}\left(k K_{n, t}\right)$. Let $u \in \mathcal{H}_{n, t, \lambda}^{\diamond}$ be a $\pi_{n, t, \lambda}^{\diamond}\left(K_{n, t}\right)$-fixed unit vector. Then $u$ belongs to the fiber $1 \cdot \mathcal{H}_{n, t, \lambda}^{\diamond}$, and $k \cdot u \in k \cdot \mathcal{H}_{n, t, \lambda}^{\diamond}$ depends only on the coset $k K_{n, t}$. Define a section

$$
\sigma_{u}: K_{n} / K_{n, t} \rightarrow \mathbb{H}_{n, t, \lambda}^{\diamond} \text { by } \sigma_{u}\left(k K_{n, t}\right)=k \cdot u \text {. }
$$

Then $\sigma_{u}$ is a $\pi_{n, t, \lambda}\left(K_{n}\right)$-invariant unit vector in the Hilbert space $\mathcal{H}_{n, t, \lambda}$. (We will also write $\varphi_{u}$ for the corresponding function $G_{n} \rightarrow \mathcal{H}_{n, t, \lambda}^{\diamond}$ such that $\varphi_{u}\left(g g_{t}\right)=$ $\pi_{n, t, \lambda}^{\diamond}\left(g_{t}\right)^{-1}\left(\varphi_{u}(g)\right)$ for $g \in G_{n}$ and $g_{t} \in G_{n, t}$. Conversely if $\sigma$ is a $\pi_{n, t, \lambda}\left(K_{n}\right)$ invariant unit vector in $\mathcal{H}_{n, t, \lambda}$, then $\sigma\left(1 K_{n, t}\right)=c u$ where $|c|=1$ by $K_{n, t}$-invariance, and then $\sigma=c \sigma_{u}$ by $K$-invariance. In summary,

Lemma 5.28 Let $t \in T$ and let $u$ be the unique (up to scalar multiple) $\pi_{n, t, \lambda}^{\diamond}\left(K_{n, t}\right)$ fixed unit vector in $\mathcal{H}_{n, t, \lambda}^{\diamond}$ Then the section $\sigma_{u}$, given by (5.27), is the unique (up to scalar multiple) $\pi_{n, t, \lambda}(K)$-fixed unit vector in $\mathcal{H}_{n, t, \lambda}$.

By Theorem 5.26 we can apply (5.21) and (5.22) to the function spaces $\mathcal{E}_{n, t, \lambda}^{\diamond}=$ $\mathcal{H}_{n, t, \lambda}^{\diamond} \otimes\left(\mathcal{H}_{n, t, \lambda}^{\diamond}\right)^{*}$ on the groups $G_{n, t}=N_{n} \rtimes K_{n, t}$. Making use of Lemma 5.28 we have

Proposition 5.29 If orthogonal projection $\mathcal{E}_{m, t, \lambda}^{\diamond} \rightarrow \mathcal{E}_{n, t, \lambda}^{\diamond}$ sends a nonzero right $K_{m, t}$-invariant function to a nonzero right $K_{n, t}$-invariant function, then orthogonal projection $\mathcal{E}_{m, t, \lambda} \rightarrow \mathcal{E}_{n, t, \lambda}$ sends a nonzero right $K_{m}$-invariant function to a nonzero right $K_{n}$-invariant function.

Vinberg classified the maximal irreducible nilpotent Gelfand pairs. See [12,13], or see [16, Table 13.4.1]. A Gelfand pair $\left(G_{n}, K_{n}\right)$ is called maximal if it is not obtained from another Gelfand pair $\left(G_{n}^{\prime}, K_{n}^{\prime}\right)$ by the construction $\left(G_{n}, K_{n}\right)=\left(G_{n}^{\prime} / C\right.$, $K_{n}^{\prime} /\left(K_{n}^{\prime} \cap C\right)$ ) for any nontrivial closed connected central subgroup $C$ of $G_{n}^{\prime}$. And $\left(G_{n}, K_{n}\right)$ is called irreducible if $\operatorname{Ad}\left(K_{n}\right)$ is irreducible on $\mathfrak{v}_{n}=\mathfrak{n}_{n} / \mathfrak{z}$. See [16] for the notation. 


\begin{tabular}{|c|c|c|c|c|c|}
\hline \multicolumn{6}{|c|}{ Maximal Irreducible Nilpotent Gelfand Pairs $\left(N_{n} \rtimes K_{n}, K_{n}\right) \quad([12,13])$} \\
\hline & Group $K_{n}$ & $\mathfrak{v}_{n}$ & $\mathfrak{z}$ & $\begin{array}{l}U(1) \text { is } \\
\text { needed if }\end{array}$ & $\begin{array}{c}\text { max } \\
\text { requires }\end{array}$ \\
\hline 1 & $S O(n)$ & $\mathbb{R}^{n}$ & Skew $\mathbb{R}^{n \times n}=\mathfrak{s o}(n)$ & & \\
\hline 2 & $\operatorname{Spin}(7)$ & $\mathbb{R}^{8}=\mathbb{O}$ & $\mathbb{R}^{7}=\operatorname{Im} \mathbb{O}$ & & \\
\hline 3 & $G_{2}$ & $\mathbb{R}^{7}=\operatorname{Im} \mathbb{O}$ & $\mathbb{R}^{7}=\operatorname{Im} \mathbb{O}$ & & \\
\hline 4 & $U(1) \cdot S O(n)$ & $\mathbb{C}^{n}$ & $\operatorname{Im} \mathbb{C}$ & & $n \neq 4$ \\
\hline 5 & $(U(1) \cdot) S U(n)$ & $\mathbb{C}^{n}$ & $\Lambda^{2} \mathbb{C}^{n} \oplus \operatorname{Im} \mathbb{C}$ & $n$ odd & \\
\hline 6 & $S U(n), n$ odd & $\mathbb{C}^{n}$ & $\Lambda^{2} \mathbb{C}^{n}$ & & \\
\hline 7 & $S U(n), n$ odd & $\mathbb{C}^{n}$ & $\operatorname{Im} \mathbb{C}$ & & \\
\hline 8 & $U(n)$ & $\mathbb{C}^{n}$ & $\operatorname{Im} \mathbb{C}^{n \times n}=\mathfrak{u}(n)$ & & \\
\hline 9 & $(U(1) \cdot) S p(n)$ & $\mathbb{H}^{n}$ & $\operatorname{Re} \mathbb{H}_{0}^{n \times n} \oplus \operatorname{Im} \mathbb{H}$ & & \\
\hline 10 & $U(n)$ & $S^{2} \mathbb{C}^{n}$ & $\mathbb{R}$ & & \\
\hline 11 & $(U(1) \cdot) S U(n), n \geqq 3$ & $\Lambda^{2} \mathbb{C}^{n}$ & $\mathbb{R}$ & $n$ even & \\
\hline 12 & $U(1) \cdot \operatorname{Spin}(7)$ & $\mathbb{C}^{8}$ & $\mathbb{R}^{7} \oplus \mathbb{R}$ & & \\
\hline 13 & $U(1) \cdot \operatorname{Spin}(9)$ & $\mathbb{C}^{16}$ & $\mathbb{R}$ & & \\
\hline 14 & $(U(1) \cdot) \operatorname{Spin}(10)$ & $\mathbb{C}^{16}$ & $\mathbb{R}$ & & \\
\hline 15 & $U(1) \cdot G_{2}$ & $\mathbb{C}^{7}$ & $\mathbb{R}$ & & \\
\hline 16 & $U(1) \cdot E_{6}$ & $\mathbb{C}^{27}$ & $\mathbb{R}$ & & \\
\hline 17 & $S p(1) \times S p(n)$ & $\mathbb{H}^{n}$ & $\operatorname{Im} \mathbb{H}=\mathfrak{s p}(1)$ & & $n \geqq 2$ \\
\hline 18 & $S p(2) \times S p(n)$ & $\mathbb{H}^{2 \times n}$ & $\operatorname{Im} \mathbb{H}^{2 \times 2}=\mathfrak{s p}(2)$ & & \\
\hline 19 & $\begin{array}{c}(U(1) \cdot) S U(m) \times S U(n) \\
m, n \geqq 3\end{array}$ & $\mathbb{C}^{m} \otimes \mathbb{C}^{n}$ & $\mathbb{R}$ & $m=n$ & \\
\hline 20 & $(U(1) \cdot) S U(2) \times S U(n)$ & $\mathbb{C}^{2} \otimes \mathbb{C}^{n}$ & $\operatorname{Im} \mathbb{C}^{2 \times 2}=\mathfrak{u}(2)$ & $n=2$ & \\
\hline 21 & $(U(1) \cdot) S p(2) \times S U(n)$ & $\mathbb{H}^{2} \otimes \mathbb{C}^{n}$ & $\mathbb{R}$ & $n \leqq 4$ & $n \geqq 3$ \\
\hline 22 & $U(2) \times S p(n)$ & $\mathbb{C}^{2} \otimes \mathbb{H}^{n}$ & $\operatorname{Im} \mathbb{C}^{2 \times 2}=\mathfrak{u}(2)$ & & \\
\hline 23 & $U(3) \times S p(n)$ & $\mathbb{C}^{3} \otimes \mathbb{H}^{n}$ & $\mathbb{R}$ & & $n \geqq 2$ \\
\hline
\end{tabular}

Splitting some cases to retain parabolicity of $\left\{K_{n}\right\}$, the strict direct systems in Table 5.30, with $\operatorname{dim} z_{n}$ bounded, are

\begin{tabular}{|r|c|c|c|c|c|}
\hline \multicolumn{5}{|c|}{ Direct Systems of Maximal Irreducible Nilpotent Gelfand Pairs $\left(N_{n} \rtimes K_{n}, K_{n}\right)$} \\
\hline \hline & Group $K_{n}$ & $\mathfrak{v}_{n}$ & $\mathfrak{z} n$ & $\begin{array}{c}\text { (1) is } \\
\text { needed if }\end{array}$ & $\begin{array}{c}\text { max } \\
\text { requires }\end{array}$ \\
\hline 4a & $U(1) \cdot S O(2 n)$ & $\mathbb{C}^{2 n}$ & $\operatorname{Im} \mathbb{C}$ & & $n \neq 2$ \\
\hline 4b & $U(1) \cdot S O(2 n+1)$ & $\mathbb{C}^{2 n+1}$ & $\operatorname{Im} \mathbb{C}$ & & \\
\hline 7 & $S U(n), n$ odd & $\mathbb{C}^{n}$ & $\operatorname{Im} \mathbb{C}$ & & \\
\hline 10 & $U(n)$ & $S^{2} \mathbb{C}^{n}$ & $\mathbb{R}$ & & \\
\hline 11 & $(U(1) \cdot) S U(n), n \geqq 3$ & $\Lambda^{2} \mathbb{C}^{n}$ & $\mathbb{R}$ & \\
\hline 17 & $S p(1) \times S p(n)$ & $\mathbb{H}^{n}$ & $\operatorname{Im} \mathbb{H}=\mathfrak{s p}(1)$ & & \\
\hline 18 & $S p(2) \times S p(n)$ & $\mathbb{H}^{2 \times n}$ & $\operatorname{Im} \mathbb{H}^{2 \times 2}=\mathfrak{s p}(2)$ & & \\
\hline 19 & $(U(1) \cdot) S U(m) \times S U(n)$ & $\mathbb{C}^{m} \otimes \mathbb{C}^{n}$ & $\mathbb{R}$ & $m=n$ & \\
\hline $20 \mathrm{a}$ & $S U(2) \times S U(n), n \geqq 3$ & $\mathbb{C}^{2} \otimes \mathbb{C}^{n}$ & $\operatorname{Im} \mathbb{C}^{2 \times 2}=\mathfrak{u}(2)$ & & \\
\hline $20 \mathrm{~b}$ & $U(2) \times S U(n)$ & $\mathbb{C}^{2} \otimes \mathbb{C}^{n}$ & $\operatorname{Im} \mathbb{C}^{2 \times 2}=\mathfrak{u}(2)$ & & \\
\hline 21 & $(U(1) \cdot) S p(2) \times S U(n)$ & $\mathbb{H}^{2} \otimes \mathbb{C}^{n}$ & $\mathbb{R}$ & $n \leqq 4$ & $n \geqq 3$ \\
\hline 22 & $U(2) \times S p(n)$ & $\mathbb{C}^{2} \otimes \mathbb{H}^{n}$ & $\operatorname{Im} \mathbb{C}^{2 \times 2}=\mathfrak{u}(2)$ & & \\
\hline 23 & $U(3) \times S p(n)$ & $\mathbb{C}^{3} \otimes \mathbb{H}^{n}$ & $\mathbb{R}$ & & \\
\hline
\end{tabular}

In each case of Table 5.31, [16, Theorem 14.4.3] says that $N_{n}$ has square integrable representations. In the cases $\operatorname{dim} \mathfrak{z}>1$ of Table 5.31 we have $K_{n}=K_{n}^{\dagger} \cdot L$ where 
the big factor $K_{n}^{\dagger}$ acts trivially on $\mathfrak{z}$ and the small factor $L$ acts on $\mathfrak{z}$ by its adjoint representation. Summarizing these observations,

Proposition 5.32 Each direct system $\left\{\left(G_{n}, K_{n}\right)\right\}$ of Table 5.31 has the properties (i) $\left\{K_{n}\right\}$ is parabolic, (ii) the $\left\{K_{n, t}\right\}$ are parabolic for each $t \in T$, and (iii) $N_{n}$ has square integrable representations.

From Theorem 5.26 and the argument of Lemma 4.20,

Corollary 5.33 Let $\left\{\left(G_{n}, K_{n}\right)\right\}$ be a direct system of Table 5.31 and let $t \in T$. Then $\pi_{n, t, \lambda}^{\diamond}$ has a nonzero $K_{n, t}$-fixed vector if and only if $\kappa_{n, t, \lambda}^{*}$ occurs as a subrepresentation of $\left.\pi_{n, t}\right|_{n, t}$, and in that case the space of $K_{n, t}$-fixed vectors has dimension 1.

Now everything goes essentially as in the Heisenberg nilmanifold cases of Sect. 4. We have isometric $G_{n}$-equivariant injections

$$
\widetilde{\zeta}_{m, n, t, \lambda}: \mathcal{E}_{n, t, \lambda}^{K_{n}} \rightarrow \mathcal{E}_{m, t, \lambda}^{K_{m}} \text { by } \widetilde{\zeta}_{m, n, t, \lambda}\left(\left(\operatorname{deg} \pi_{n, t, \lambda}\right)^{1 / 2} f\right)=c_{m, n, t, \lambda}\left(\operatorname{deg} \pi_{m, t, \lambda}\right)^{1 / 2} f
$$

where as in (4.24), $c_{m, n, t, \lambda}$ is the ratio (4.23) of lengths of $K_{n}, K_{m}$ fixed unit vectors. Integrating on $t$ and summing $\lambda$ gives isometric $G_{n}$-equivariant injections $\widetilde{\zeta}_{m, n}$ : $L^{2}\left(G_{n} / K_{n}\right) \rightarrow L^{2}\left(G_{m} / K_{m}\right)$, as follows.

Theorem 5.35 For each of the direct systems of Table 5.31 denote $G=\underset{\sim}{\lim }\left\{G_{n}\right\}$, $N=\underline{\lim } N_{n}$ and $K=\lim _{\longrightarrow}\left\{K_{n}\right\}$. Note $G=N \rtimes K$. Then $\left\{L^{2}\left(G_{n} / K_{n}\right), \widetilde{\widetilde{\zeta}_{m, n}}\right\}$ is a

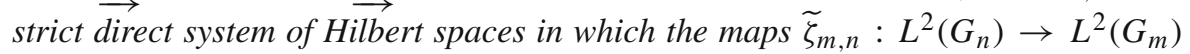
are $G_{n}$-equivariant injections. That gives us the left regular representation of $G$ on the Hilbert space $L^{2}(G):=\lim _{\longrightarrow}\left\{L^{2}\left(G_{n}\right), \zeta_{m, n}\right\}$. Further, that left/right regular representation is the multiplicity-free $\sum_{\lambda} \int_{\mathrm{Ad}^{*}(L) \backslash \mathfrak{z}^{*}}\left(\pi_{\operatorname{Ad}^{*}(k) t, \operatorname{Ad}^{*}(k) \lambda} \otimes \pi_{\mathrm{Ad}^{*}(k) t, \operatorname{Ad}^{*}(k) \lambda}\right) d k$ where $\pi_{t, \lambda}:=\lim _{\longrightarrow} \pi_{n, t, \lambda}$.

Similarly using $w_{m, \lambda}=c_{m, n, t, \lambda} w_{n, \lambda}+x$ with $x \perp \mathcal{E}_{n, t, \lambda}$ with $0<c_{n, t, \lambda} \leqq 1$,

$$
\mathcal{A}\left(G_{n} / K_{n}\right):=\mathcal{A}\left(G_{n}\right)^{K_{n}}=\mathcal{A}\left(G_{n}\right) \cap \mathcal{E}_{n, t, \lambda}^{K_{n}}
$$

leading to direct systems and their limits by assembling the maps

$$
v_{m, n, t, \lambda}: \mathcal{A}\left(\pi_{n, t, \lambda}\right)^{K_{n}} \hookrightarrow \mathcal{A}\left(\pi_{m, t, \lambda}\right)^{K_{m}} \text { by } f_{u, v_{n}, n} \mapsto f_{u, v_{m}, m} \text { where } p_{m, n, t, \lambda}\left(v_{m}\right)=v_{n}
$$

Then we have direct systems and their limits

$$
\begin{aligned}
\mathcal{A}(G) & =\underset{\lim }{\longrightarrow}\left\{\mathcal{A}\left(G_{n}\right), \zeta_{m, n}\right\} \text { where } \zeta_{m, n, t, \lambda}: \mathcal{E}_{n, t, \lambda} \hookrightarrow \mathcal{E}_{m, t, \lambda}(5.21), \text { and } \\
\mathcal{A}(G / K) & \left.=\lim _{\longrightarrow}\left\{\mathcal{A}\left(G_{n}\right) / K_{n}\right), v_{m, n}\right\} \text { where } v_{m, n, t, \lambda}: \mathcal{E}_{n, t, \lambda}^{K_{n}} \hookrightarrow \mathcal{E}_{m, t, \lambda}^{K_{m}} \text { (5.37). }
\end{aligned}
$$

As before, each $\mathcal{A}\left(G_{n} / K_{n}\right)$ is dense in $L^{2}\left(G_{n} / K_{n}\right)$, and we pass this comparison to the limit with the maps 


$$
\tilde{\eta}_{n, t, \lambda}: \mathcal{A}\left(\pi_{n, t, \lambda}\right)^{K_{n}} \rightarrow \mathcal{H}_{\pi_{n, t, \lambda}} \otimes\left(w_{n, t, \lambda} * \mathbb{C}\right) \text { by } f \mapsto c_{n, 1, t, \lambda} \sqrt{\operatorname{deg} \pi_{n, t, \lambda}} f .
$$

We conclude

Proposition 5.40 The $\widetilde{\eta}_{n, t, \lambda}$ satisfy $\left(\widetilde{\eta}_{m, t, \lambda} \circ v_{m, n, t, \lambda}\right)(f)=\left(\widetilde{\zeta}_{m, n, \lambda} \circ \widetilde{\eta}_{n, t, \lambda}\right)(f)$ for $f \in$ $\mathcal{A}\left(\pi_{n, t, \lambda}\right)^{K_{n}}$ Thus they inject the direct system $\left\{\mathcal{A}\left(G_{n} / K_{n}\right), v_{m, n}\right\}$ of regular functions into the direct system $\left\{L^{2}\left(G_{n} / K_{n}\right), \widetilde{\zeta}_{m, n}\right\}$. That map of direct systems defines a $G$-equivariant injection $\tilde{\eta}: \mathcal{A}(G / K) \rightarrow L^{2}(G / K)$ with dense image. In particular $\tilde{\eta}$ defines a pre Hilbert space structure on $\mathcal{A}(G / K)$ with completion isometric to $L^{2}(G / K)$.

\subsection{Reducible quotients}

There are more many strict direct sequences of nilmanifold Gelfand pairs $\left(G_{n}, K_{n}\right)$, e.g. those for which the action of $K_{n}$ on $\mathfrak{n}_{n} / \mathfrak{z}_{n}$ is reducible. These $\left(G_{n} / K_{n}\right)$ are constructed from certain basic ones that satisfy several technical conditions (indecomposable, principal, maximal and $S p(1)$-saturated). See [16,19-21] and [18]. The basic such direct systems, with $K_{n}$ reducible on $\mathfrak{n}_{n} / \mathfrak{z}_{n}$, $\operatorname{dim} \mathfrak{z}_{n}$ bounded and $\left\{K_{n}\right\}$ parabolic, are tabulated in [18, Table 9.15] as follows. Here the numbering comes from [18, Table 9.14], $N_{\ell}=N_{\ell}^{\prime} \times Z_{\ell}$ with $Z_{\ell}$ central and maximal for that, $G_{\ell}=N_{\ell} \rtimes K_{\ell}$ and $G_{\ell}^{\prime}=N_{\ell}^{\prime} \rtimes$ $K_{\ell}$. The generalized Heisenberg algebra $\mathfrak{h}_{n, \mathbb{F}}$ is $\operatorname{Im} \mathbb{F}+\mathbb{F}^{n}$ with $\left[(z, w),\left(z^{\prime}, w^{\prime}\right)\right]=$ $\left(z+z^{\prime}+\operatorname{Im}\left\langle w, w^{\prime}\right\rangle, w+w^{\prime}\right)$ where $\mathbb{F}=\mathbb{C}, \mathbb{H}$ (quaternions) or $\mathbb{O}$ (octonions).

\begin{tabular}{|c|c|c|c|c|c|}
\hline \multicolumn{6}{|c|}{ Strict Direct Systems $\left\{\left(G_{\ell}, K_{\ell}\right)\right\}$ and $\left\{\left(G_{\ell}^{\prime}, K_{\ell}^{\prime}\right)\right\}$ of Gelfand Pairs with $\operatorname{dim}_{\mathfrak{z}_{\ell}^{\prime}}$ Bounded } \\
\hline & Group $K_{\ell}$ & $K_{\ell}-$ module $\mathfrak{v}_{\ell}$ & $\mathfrak{z}_{\ell}^{\prime}=\left[\mathfrak{n}_{\ell}, \mathfrak{n}_{\ell}\right]$ & $\mathfrak{z}_{\ell}^{\prime \prime}$ & Algebra $\mathfrak{n}_{\ell}^{\prime}$ \\
\hline 1 & $U(n)$ & $\mathbb{C}^{n}$ & $\mathbb{R}$ & $\mathfrak{s u}(n)$ & $\mathfrak{h}_{n ; \mathbb{C}}$ \\
\hline 3 & $U(1) \times U(n)$ & $\mathbb{C}^{n} \oplus \Lambda^{2} \mathbb{C}^{n}$ & $\mathbb{R} \oplus \mathbb{R}$ & 0 & $\mathfrak{h}_{n ; \mathbb{C}} \oplus \mathfrak{h}_{n(n-1) / 2 ; \mathbb{C}}$ \\
\hline 6 & $S(U(4) \times U(m))$ & $\mathbb{C}^{4 \times m}$ & $\mathbb{R}$ & $\mathbb{R}^{6}$ & $\mathfrak{h}_{4 m ; \mathbb{C}}$ \\
\hline 7 & $U(m) \times U(n)$ & $\mathbb{C}^{m \times n} \oplus \mathbb{C}^{m}$ & $\mathbb{R} \oplus \mathbb{R}$ & 0 & $\mathfrak{h}_{m n ; \mathbb{C}} \oplus \mathfrak{h}_{m ; \mathbb{C}}$ \\
\hline 8 & $U(1) \times S p(n) \times U(1)$ & $\mathbb{C}^{2 n} \oplus \mathbb{C}^{2 n}$ & $\mathbb{R} \oplus \mathbb{R}$ & 0 & $\mathfrak{h}_{2 n ; \mathbb{C}} \oplus \mathfrak{h}_{2 n ; \mathbb{C}}$ \\
\hline 9 & $S p(1) \times S p(n) \times U(1)$ & $\mathbb{H}^{n} \oplus \mathbb{H}^{n}$ & $\operatorname{Im} \mathbb{H} \oplus \mathbb{R}$ & 0 & $\mathfrak{h}_{n ; \mathbb{H}} \oplus \mathfrak{h}_{2 n ; \mathbb{C}}$ \\
\hline 10 & $S p(1) \times S p(n) \times S p(1)$ & $\mathbb{H}^{n} \oplus \mathbb{H}^{n}$ & $\operatorname{Im} \mathbb{H} \oplus \operatorname{Im} \mathbb{H}$ & 0 & $\mathfrak{h}_{n ; \mathbb{H}} \oplus \mathfrak{h}_{n ; \mathbb{H}}$ \\
\hline $11 \mathrm{a}$ & $S p(n) \times S p(1) \times S p(m)$ & $\mathbb{H}^{n}$ & $\operatorname{Im} \mathbb{H}$ & $\mathbb{H}^{n \times m}$ & $\mathfrak{h}_{n ; \mathbb{H}}$ \\
\hline $11 \mathrm{~b}$ & $S p(n) \times U(1) \times S p(m)$ & $\mathbb{H}^{n}$ & $\operatorname{Im} \mathbb{H}$ & $\mathbb{H}^{n \times m}$ & $\mathfrak{h}_{n ; \mathbb{H}}$ \\
\hline $11 \mathrm{c}$ & $S p(n) \times\{1\} \times S p(m)$ & $\mathbb{H}^{n}$ & $\operatorname{Im} \mathbb{H}$ & $\mathbb{H}^{n \times m}$ & $\mathfrak{h}_{n ; \mathbb{H}}$ \\
\hline $18 \mathrm{a}$ & $S U(n) \times S U(2)$ & $\mathbb{C}^{n \times 2}$ & $\mathbb{R}$ & $\mathfrak{s u ( 2 )}$ & $\mathfrak{h}_{2 n ; \mathbb{C}}$ \\
\hline $18 \mathrm{~b}$ & $U(n) \times S U(2)$ & $\mathbb{C}^{n \times 2}$ & $\mathbb{R}$ & $\mathfrak{s u ( 2 )}$ & $\overline{\mathfrak{h}_{2 n ; \mathbb{C}}}$ \\
\hline $18 \mathrm{c}$ & $U(1) S p\left(\frac{n}{2}\right) \times S U(2)$ & $\mathbb{C}^{n \times 2}$ & $\mathbb{R}$ & $\mathfrak{s u}(2)$ & $\mathfrak{h}_{2 n ; \mathbb{C}}$ \\
\hline $19 a$ & $S U(n) \times U(2)$ & $\mathbb{C}^{n \times 2} \oplus \mathbb{C}^{2}$ & $\mathbb{R} \oplus \mathbb{R}$ & 0 & $\mathfrak{h}_{2 n ; \mathbb{C}} \oplus \mathfrak{h}_{2 ; \mathbb{C}}$ \\
\hline $19 \mathrm{~b}$ & $U(n) \times U(2)$ & $\mathbb{C}^{n \times 2} \oplus \mathbb{C}^{2}$ & $\mathbb{R} \oplus \mathbb{R}$ & 0 & $\mathfrak{h}_{2 n ; \mathbb{C}} \oplus \mathfrak{h}_{2 ; \mathbb{C}}$ \\
\hline $19 \mathrm{c}$ & $U(1) S p\left(\frac{n}{2}\right) \times U(2)$ & $\mathbb{C}^{n \times 2} \oplus \mathbb{C}^{2}$ & $\mathbb{R} \oplus \mathbb{R}$ & 0 & $\mathfrak{h}_{2 n ; \mathbb{C}} \oplus \mathfrak{h}_{2 ; \mathbb{C}}$ \\
\hline $20 a a$ & $S U(n) \times S U(2) \times S U(m)$ & $\mathbb{C}^{n \times 2} \oplus \mathbb{C}^{2 \times m}$ & $\mathbb{R} \oplus \mathbb{R}$ & 0 & $\mathfrak{h}_{2 n ; \mathbb{C}} \oplus \mathfrak{h}_{2 m ; \mathbb{C}}$ \\
\hline $20 \mathrm{ab}$ & $S U(n) \times S U(2) \times U(m)$ & $\mathbb{C}^{n \times 2} \oplus \mathbb{C}^{2 \times m}$ & $\mathbb{R} \oplus \mathbb{R}$ & 0 & $\mathfrak{h}_{2 n ; \mathbb{C}} \oplus \mathfrak{h}_{2 m ; \mathbb{C}}$ \\
\hline $20 \mathrm{ac}$ & $S U(n) \times S U(2) \times U(1) S p\left(\frac{m}{2}\right)$ & $\mathbb{C}^{n \times 2} \oplus \mathbb{C}^{2 \times m}$ & $\mathbb{R} \oplus \mathbb{R}$ & 0 & $\mathfrak{h}_{2 n ; \mathbb{C}} \oplus \mathfrak{h}_{2 m ; \mathbb{C}}$ \\
\hline 20ba & $U(n) \times S U(2) \times S U(m)$ & $\mathbb{C}^{n \times 2} \oplus \mathbb{C}^{2 \times m}$ & $\mathbb{R} \oplus \mathbb{R}$ & 0 & $\mathfrak{h}_{2 n ; \mathbb{C}} \oplus \mathfrak{h}_{2 m ; \mathbb{C}}$ \\
\hline $20 \mathrm{bb}$ & $U(n) \times S U(2) \times U(m)$ & $\mathbb{C}^{n \times 2} \oplus \mathbb{C}^{2 \times m}$ & $\mathbb{R} \oplus \mathbb{R}$ & 0 & $\mathfrak{h}_{2 n ; \mathbb{C}} \oplus \mathfrak{h}_{2 m ; \mathbb{C}}$ \\
\hline $20 \mathrm{bc}$ & $U(n) \times S U(2) \times U(1) S p\left(\frac{m}{2}\right)$ & $\mathbb{C}^{n \times 2} \oplus \mathbb{C}^{2 \times m}$ & $\mathbb{R} \oplus \mathbb{R}$ & 0 & $\mathfrak{h}_{2 n ; \mathbb{C}} \oplus \mathfrak{h}_{2 m ; \mathbb{C}}$ \\
\hline $20 \mathrm{ca}$ & $U(1) S p\left(\frac{n}{2}\right) \times S U(2) \times S U(m)$ & $\mathbb{C}^{n \times 2} \oplus \mathbb{C}^{2 \times m}$ & $\mathbb{R} \oplus \mathbb{R}$ & 0 & $\mathfrak{h}_{2 n ; \mathbb{C}} \oplus \mathfrak{h}_{2 m ; \mathbb{C}}$ \\
\hline $20 \mathrm{cb}$ & $U(1) S p\left(\frac{n}{2}\right) \times S U(2) \times U(m)$ & $\mathbb{C}^{n \times 2} \oplus \mathbb{C}^{2 \times m}$ & $\mathbb{R} \oplus \mathbb{R}$ & 0 & $\mathfrak{h}_{2 n ; \mathbb{C}} \oplus \mathfrak{h}_{2 m ; \mathbb{C}}$ \\
\hline $20 \mathrm{cc}$ & $\left.U(1) S p\left(\frac{n}{2}\right) \times S U(2) \times U(1) S p\left(\frac{m}{2}\right)\right\}$ & $\mathbb{C}^{n \times 2} \oplus \mathbb{C}^{2 \times m}$ & $\mathbb{R} \oplus \mathbb{R}$ & 0 & $\mathfrak{h}_{2 n ; \mathbb{C}} \oplus \mathfrak{h}_{2 m ; \mathbb{C}}$ \\
\hline $21 \mathrm{a}$ & $S U(n) \times S U(2) \times U(4)$ & $\mathbb{C}^{n \times 2} \oplus \mathbb{C}^{2 \times 4}$ & $\mathbb{R} \oplus \mathbb{R}$ & $\mathbb{R}^{6}$ & $\mathfrak{h}_{2 n ; \mathbb{C}} \oplus \mathfrak{h}_{8 ; \mathbb{C}}$ \\
\hline $21 b$ & $U(n) \times S U(2) \times U(4)$ & $\mathbb{C}^{n \times 2} \oplus \mathbb{C}^{2 \times 4}$ & $\mathbb{R} \oplus \mathbb{R}$ & $\mathbb{R}^{6}$ & $\mathfrak{h}_{2 n ; \mathbb{C}} \oplus \mathfrak{h}_{8 ; \mathbb{C}}$ \\
\hline $21 \mathrm{c}$ & $U(1) S p\left(\frac{n}{2}\right) \times S U(2) \times U(4)$ & $\mathbb{C}^{n \times 2} \oplus \mathbb{C}^{2 \times 4}$ & $\mathbb{R} \oplus \mathbb{R}$ & $\mathbb{R}^{6}$ & $\mathfrak{h}_{2 n ; \mathbb{C}} \oplus \mathfrak{h}_{8 ; \mathbb{C}}$ \\
\hline
\end{tabular}


One obtains the structure of $L^{2}(G / K)$ and $\mathcal{A}(G / K)$ for the cases of Table 5.41 by a straightforward modification of the considerations involved for the direct systems of Table 5.31. We leave the details to the reader.

Open Access This article is distributed under the terms of the Creative Commons Attribution Noncommercial License which permits any noncommercial use, distribution, and reproduction in any medium, provided the original author(s) and source are credited.

\section{References}

1. Benson, C., Jenkins, J., Ratcliff, G.: On Gelfand pairs associated with solvable Lie groups. Trans. Am. Math. Soc. 321, 85-116 (1990)

2. Carcano, G.: A commutativity condition for algebras of invariant functions. Boll. Un. Mat. Italiano 7, 1091-1105 (1987)

3. Chevalley, C.: Theory of Lie Groups, I. Princeton University Press, Princeton (1946)

4. Dimitrov, I., Penkov, I., Wolf, J.A.: A Bott-Borel-Weil theory for direct limits of algebraic groups. Am. J. Math. 124, 955-998 (2002)

5. Kač, V.: Some remarks on nilpotent orbits. J. Algebra 64, 190-213 (1980)

6. Lauret, J.: Modified $H$-type groups and symmetric-like Riemannian spaces. Differ. Geom. Appl. 10, 121-143 (1999)

7. Lichtenstein, W.: Qualitative behavior of special functions on compact symmetric spaces. J. Funct. Anal. 34, 433-455 (1979)

8. Moore, C.C., Wolf, J.A.: Square integrable representations of nilpotent groups. Trans. Am. Math. Soc. 185, 445-462 (1973)

9. Natarajan, L., Rodríguez-Carrington, E., Wolf, J.A.: Locally convex Lie groups. Nova J. Algebra Geom. 2, 59-87 (1993)

10. Palais, R.S.: The classification of $G$-Spaces. Memoirs Am. Math. Soc. 36 (1960)

11. Tannaka, T.: Über den Dualitätssatz der nichtkommutativen topologischen Gruppen. Tohoku Math. J. 45, 1-12 (1938)

12. Vinberg, E.B.: Commutative homogeneous spaces and co-isotropic symplectic actions. Russ. Math. Surv. 56, 1-60 (2001)

13. Vinberg, E.B.: Commutative homogeneous spaces of Heisenberg type. Trans. Moscow Math. Soc. 64, 45-78 (2003)

14. Wolf, J.A.: Representations of certain semidirect product groups. J. Funct. Anal. 19, 339-372 (1975)

15. Wolf, J.A.: Direct limits of principal series representations. Compos. Math. 141, 1504-1530 (2005)

16. Wolf, J.A.: Harmonic Analysis on Commutative Spaces. Mathematical Surveys and Monographs, vol. 142. American Mathematical Society, Providence (2007)

17. Wolf, J.A.: Infinite dimensional multiplicity free spaces I: Limits of compact commutative spaces. In: Neeb, K.-H., Pianzola, A. (eds.) Developments and Trends in Infinite Dimensional Lie Theory. Birkhäuser, Basel (2010, to appear) \{arXiv:0801.3869 (math.RT, math.DG)\}

18. Wolf, J.A.: Infinite dimensional multiplicity free spaces II: Limits of commutative nilmanifolds. In: Proceedings of the Sixth Workshop on Lie Theory and Geometry, Contemporary Mathematics, vol. 491, pp. 179-208 (2009). \{arXiv:0801.3866 (math.RT, math.DG)\}

19. Yakimova, O.S.: Weakly symmetric Riemannian manifolds with reductive isometry group. Math. USSR Sbornik 195, 599-614 (2004)

20. Yakimova, O.S.: Gelfand pairs, Bonner Math. Schriften (Universität Bonn), vol. 374 (2005)

21. Yakimova, O.S.: Principal Gelfand pairs. Transform. Groups 11, 305-335 (2006)

22. Yang, C.T.: On a problem of montgomery. Proc. Am. Math. Soc. 8, 255-257 (1957) 